Florida International University FIU Digital Commons

FIU Electronic Theses and Dissertations

University Graduate School

$11-6-2015$

\title{
Regulatory mechanisms of Leishmania Aquaglyceroporin AQP1
}

Mansi Sharma

Florida International University, sharma.mansi30@gmail.com

DOI: $10.25148 /$ etd.FIDC000197

Follow this and additional works at: https:// digitalcommons.fiu.edu/etd

Part of the Parasitology Commons

\section{Recommended Citation}

Sharma, Mansi, "Regulatory mechanisms of Leishmania Aquaglyceroporin AQP1" (2015). FIU Electronic Theses and Dissertations. 2300.

https://digitalcommons.fiu.edu/etd/2300

This work is brought to you for free and open access by the University Graduate School at FIU Digital Commons. It has been accepted for inclusion in FIU Electronic Theses and Dissertations by an authorized administrator of FIU Digital Commons. For more information, please contact dcc@fiu.edu. 


\section{FLORIDA INTERNATIONAL UNIVERSITY}

Miami, Florida

\section{REGULATORY MECHANISMS OF LEISHMANIA AQUAGLYCEROPORIN AQP1}

A dissertation submitted in partial fulfillment of the requirements for the degree of DOCTOR OF PHILOSOPHY

in

BIOLOGY

by

Mansi Sharma

2015 
To: Dean Michael R. Heithaus

College of Arts and Sciences

This dissertation, written by Mansi Sharma, and entitled, Regulatory Mechanisms of Leishmania Aquaglyceroporin AQP1, having been approved in respect to style and intellectual content, is referred to you for judgment.

We have read this dissertation and recommend that it be approved.

$\begin{array}{r}\hline \text { Lidia Kos } \\ \hline \text { Kathleen Rein } \\ \hline \text { Fernando Noriega } \\ \hline \text { Helen Tempest } \\ \hline \text { Rita Mukhopadhyay, Major Professor }\end{array}$

Date of Defense: November 6, 2015

The dissertation of Mansi Sharma is approved.

Dean Michael R. Heithaus College of Arts and Sciences

Dean Lakshmi N. Reddi University Graduate School

Florida International University, 2015 
(C) Copyright 2015 by Mansi Sharma

All rights reserved. 


\section{DEDICATION}

I dedicate this dissertation to my late grandfather Dr. S.K. Sharma and Dr. V.D. Sharma. Both of you will always be my inspiration. 


\section{ACKNOWLEDGMENTS}

This dissertation would not have been possible without the support of many people. I would like to thank my mentor Dr. Rita Mukhopadhyay for giving me an opportunity to work under her immense and incredible guidance. It was her support throughout the four years, which helped me in surviving the graduate school. Apart from being a good mentor, she always cared and protected me like her own child. Thank you Rita for everything. I would like to thank all my committee members Dr. Lidia Kos, Dr. Kathleen Rein, Dr. Fernando Noriega, and Dr. Helen Tempest for helping me in developing a critical thought process. I am really thankful to all of you for all the valuable advice you gave me since the qualifying examination and during all the committee meetings. Doing research without money is not possible. I would like to thank the Department of Biology for providing me teaching assistantship for a year and Herbert Wertheim College of Medicine for the Research assistantship. I also want to thank MBRS RISE for the Biomedical Research Initiative award for two years in a row. This award was really helpful in carrying out my research project. My sincere thanks to Dr. Goutam Mandal for teaching me everything in the lab and guiding me out throughout these four years. I would like to thank Julie for being an elder sister to me. Thank you for helping me in my experiments even during weekends. It is hard to do experiments without glasswares and clean lab. Christian McDonald and Yeily Hernandez, I have no words to describe how thankful I am for the lab maintenance you guys did for the past two years persistently and efficiently. Thank you guys. I would like to thank Dr. Hiranmoy Bhattacharjee for all the helpful advice, which helped me in getting my first author publication. I would like to also thank Dr. Barry Rosen and his team for providing 
me with the lab instruments needed for my research projects. More specifically, I want to acknowledge Dr. Charles Packianathan for helping me whenever I needed to use the lab instruments.

Completion of this humongous task would not have been possible also without my lovely and supportive parents, Mr. Rakesh Sharma and Dr. Nidhi Sharma. They always encouraged me, motivated me and never made me feel weak. I am really fortunate to have parents like you. Thank you for supporting me in fulfilling my dream. I would like to thank my brother, Vardan for being a friend rather than an annoying younger brother. I would like to thank all my family members for their support and love.

Life is beyond imagination without friends. I would like to thank Kanika, Rajat and Vipin for selfless 15 years of friendship and being with me in good and bad times. My special thanks goes to Vaibhav for your incredible and immeasurable support throughout these years. This would not have been possible without you. Away from home, roommates play the most important role. Thank you Priyanka for being an awesome roomie and bearing my mood swings. My special and heartiest thanks goes to my FIU friends. I would like to thank Pratik, Gorakh and Jitesh for being amazing friends since the day I joined FIU. I would like to thank Parul, Adwait, Sharief, Mayur, Shashank, Dr. Pandiaraj, Dr. Vidya Sagar, Dr. Rahul Jayant, Supurna and Ketaki for all the selfless support and friendship. Thanks to all my FIU friends for all our fun-filled vacations and our time spent together, a major stress reliever during graduate school. Special thanks to Dr. Deepak Balasubramanian for being a lovely friend, and a great support in all scientific discussions outside the research project and for inspiring me to do good science. Although my journey here has come to an end, I will forever be grateful 
for how each one of you inspired, helped and guided me in one way or another to the achievement of my dream of becoming a good scientist. 


\title{
ABSTRACT OF THE DISSERTATION
}

\section{REGULATORY MECHANISMS OF LEISHMANIA AQUAGLYCEROPORIN AQP1}

\author{
by
}

\author{
Mansi Sharma \\ Florida International University, 2015 \\ Miami, Florida \\ Professor Rita Mukhopadhyay, Major Professor
}

Pentavalent antimonials $[\mathrm{Sb}(\mathrm{V})]$ are the primary drug of choice against all forms of leishmaniasis. Emergence of antimony unresponsiveness is a major issue. There is a dire need of understanding antimony resistance mechanisms in Leishmania. One important mechanism is the down regulation of the trivalent antimony [Sb(III)] (the active form of $\mathrm{Sb}(\mathrm{V}))$ uptake system. To date, Leishmania aquaglyceroporin AQP1 is the only reported facilitator of Sb(III). Leishmania do not have promoters. They primarily regulate their genes at post-transcriptional and/or post-translational levels. We reported that mitogen activated protein kinase 2 (MPK2) positively regulated AQP1 stability through the phosphorylation of the threonine 197 (T197) residue of AQP1. The goal of this study was to elucidate the regulatory mechanism(s) of AQP1 in Leishmania in order to advance our understanding about the physiological role(s) of AQP1 in Leishmania biology. When Leishmania promastigotes were treated with the proteasome inhibitor MG132, SbIII accumulation was increased due to upregulation of AQP1. Alteration of lysine 12 of AQP1 to either alanine or arginine improved protein stability. Cells coexpressing a dominant-negative MPK2 mutant exhibited severely reduced AQP1 expression, which was reversed upon addition of MG132. Interestingly, the dominant- 
negative MPK2 mutant could not destabilize either AQP1к12A /AQP1к12R. Stabilization of AQP1 by MPK2 led to its relocalization from the flagellum to the entire surface of the parasite. Both altered AQP1 K12A and AQP1 K12R were restricted to the flagellum only. The data demonstrated that lysine12 was targeted for AQP1 proteasomal degradation playing an integral role in subcellular localization of AQP1 as well as its interaction with MPK2.

This study also demonstrated that the stability of AQP1 mRNA in different Leishmania species was regulated by their respective 3'-untranslated regions. Cutaneous leishmaniasis causing species accumulated more antimonite and therefore, exhibited higher sensitivity to antimonials than species responsible for visceral leishmaniasis. This species-specific differential sensitivity to antimonite was found to be directly proportional to the expression levels of AQP1 mRNA. The differential regulation of AQP1 mRNA explained the distinct antimonial sensitivity of each species. This study will help us to identify new drugs for treatment in the future and also lead to a novel understanding of parasite biology aspects such as integral membrane protein trafficking and regulation. 


\section{TABLE OF CONTENTS}

CHAPTER $\quad$ PAGE

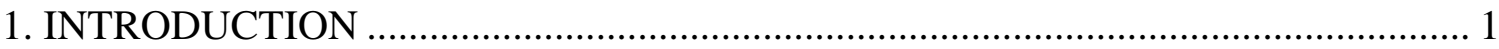

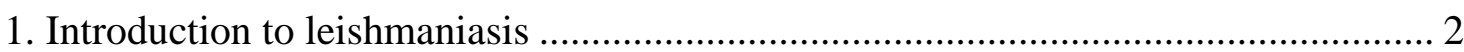

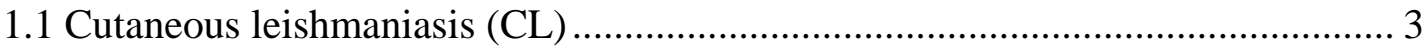

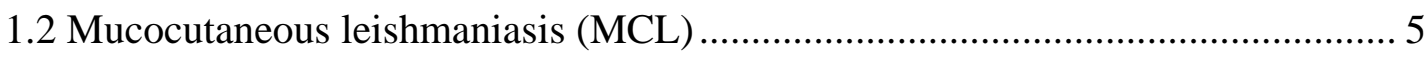

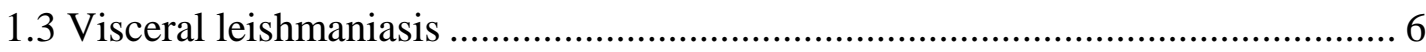

1.4 Life cycle of Leishmania .................................................................................. 7

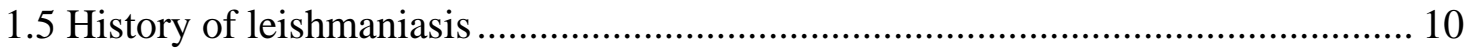

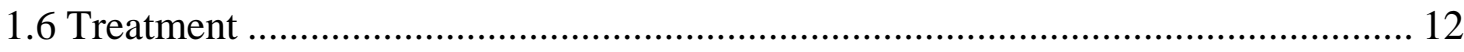

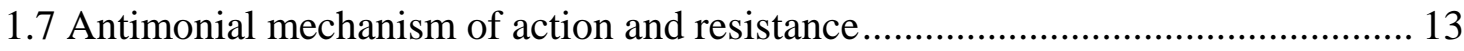

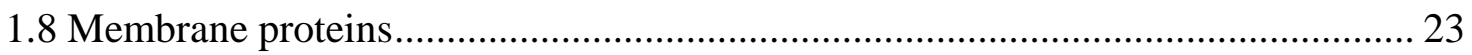

1.8.1. Peripheral membrane protein or extrinsic protein ........................................... 23

1.8.2. Integral membrane proteins or intrinsic proteins............................................. 24

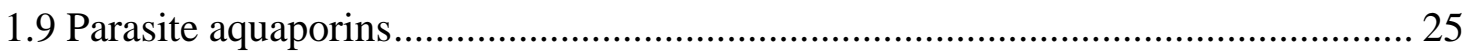

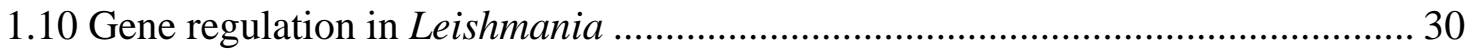

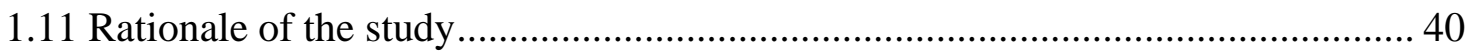

1.11.1 Deciphering the mechanism (s) of regulation of AQP1 at the

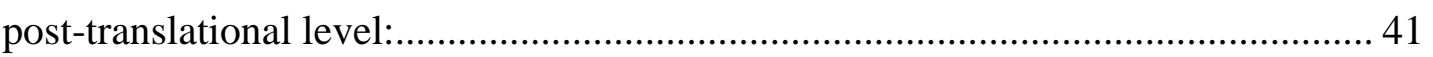

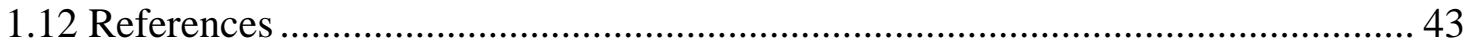

2. FUNCTIONAL ROLE OF LYSINE 12 IN LEISHMANIA MAJOR AQP1 ................. 53

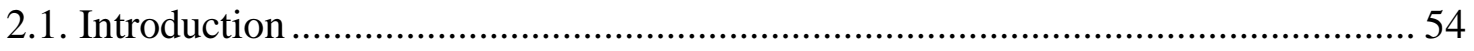

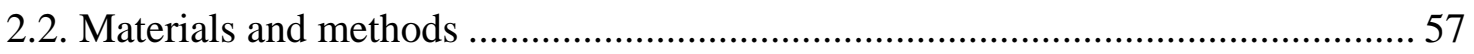

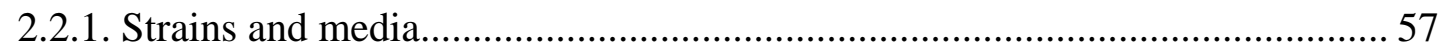

2.2.2. Oligonucleotide-directed mutagenesis ........................................................... 59

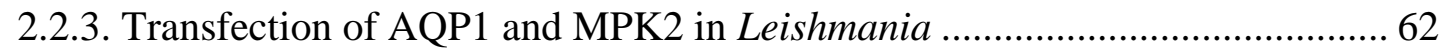

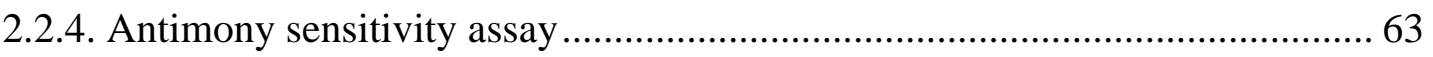

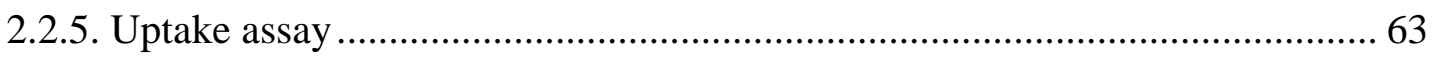

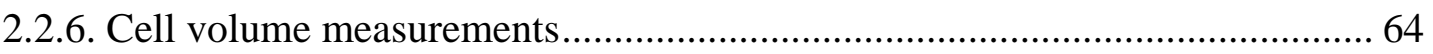

2.2.7. Flagella and pellicular membrane isolation and Immunoblotting .................... 65

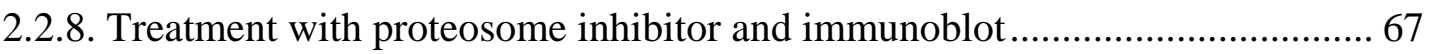

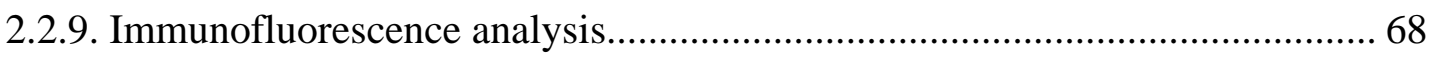

2.2.10. Determination of wild type and altered AQP1 half-lives from different

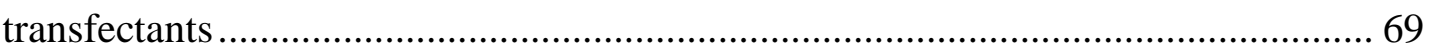

2.2.11. Determination of AQP1 mRNA levels from different transfectants .............. 69

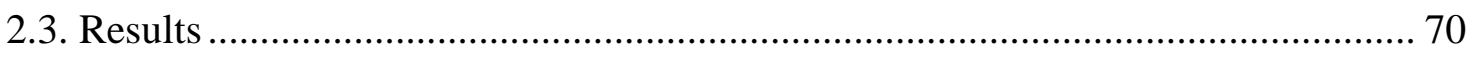

2.3.1. AQP1 degradation is mediated by the proteasome........................................... 70 
2.3.2. Lysine 12 as the putative ubiquitination site of AQP1

2.3.3. Alteration of lysine 12 of AQP1 confers efficient osmoregulation in

Leishmania. 78

2.3.4. Alteration of lysine 12 of AQP1 confers antimonite sensitivity

in Leishmania 80

2.3.5. Lysine 12 is responsible for MPK2 $442 \mathrm{~A}$ mediated degradation of AQP1 ....... 82

2.3.6. Reversal of K42AMPK2 mediated destabilization of AQP1 by alteration of lysine 12 takes place by increasing its half life..................................................... 85

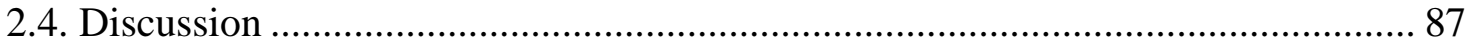

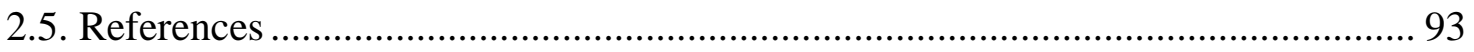

\section{ROLE OF 3'-UTR IN POST-TRANSCRIPTIONAL REGULATION OF} AQP1 IN DIFFERENT SPECIES OF LEISHMANIA .............................................. 97

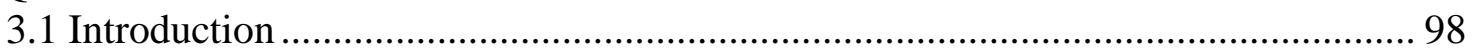

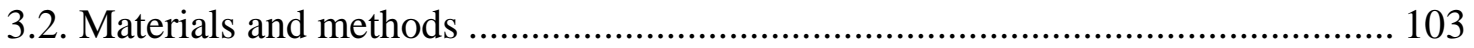

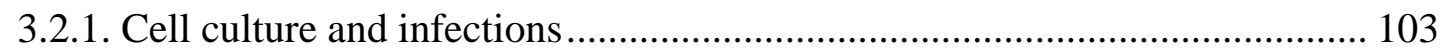

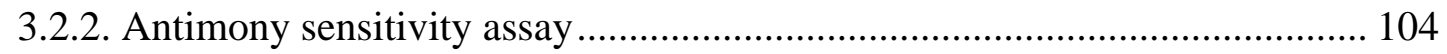

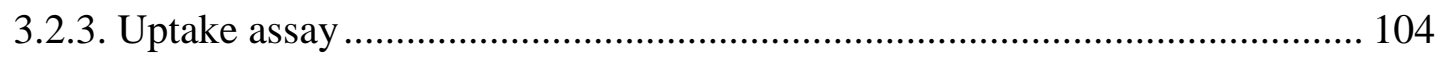

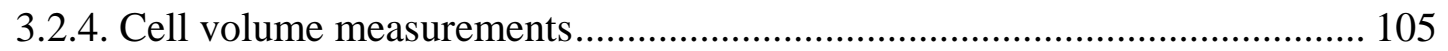

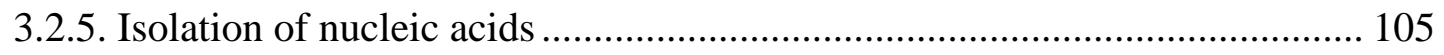

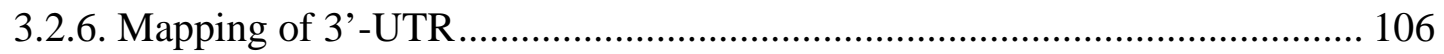

3.2.7. Plasmid construction and transfection.......................................................... 106

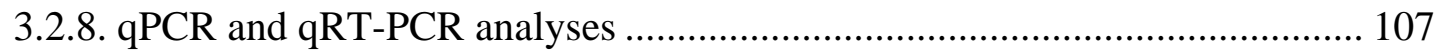

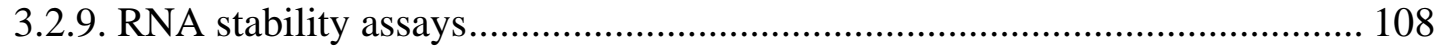

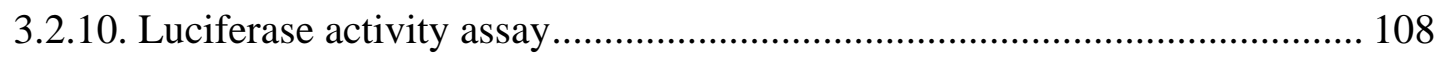

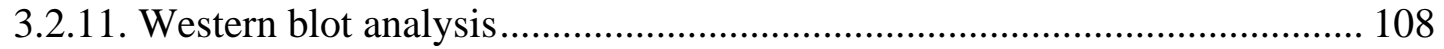

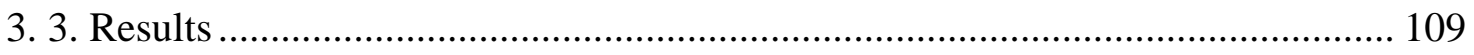

3.3.1. Intrinsic species-specific antimonial resistance in Leishmania ..................... 109

3.3.2. Cutaneous leishmaniasis species accumulate more $\mathrm{Sb}(\mathrm{III})$ than

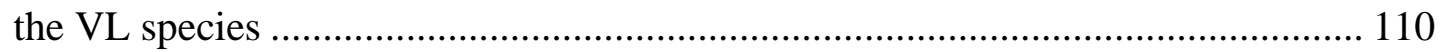

3.3.4. AQP1 mRNA is more stable in the Cutaneous leishmaniasis species ........... 113

3.3.5. Cloning of 3'-UTRs of AQP1 mRNA from different species........................ 115

3.3.6. Role of the 3'-UTR in species-specific stability of AQP1 mRNA................. 115

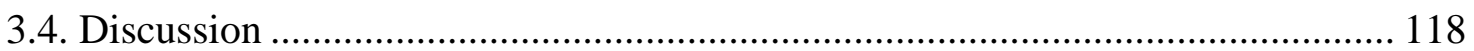

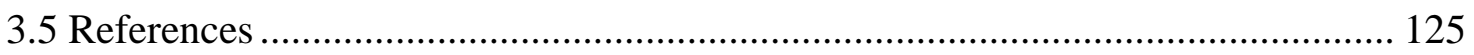

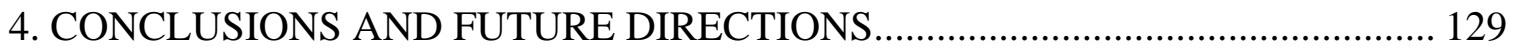

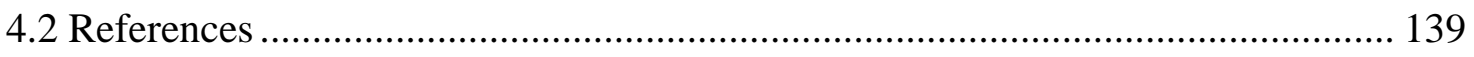




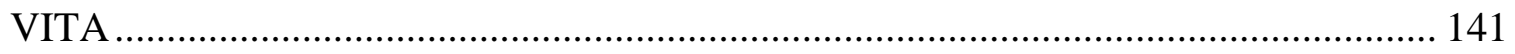




\section{LIST OF FIGURES}

FIGURE

PAGE

1. Geographical distribution of different types of leishmaniasis ..................................2

2. Cutaneous form of leishmaniasis depicting (A) localized cutaneous lesions

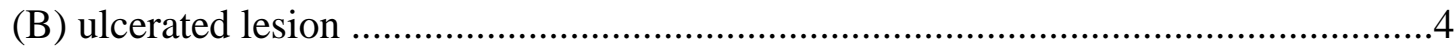

3. Patient suffering from Espundia caused by L. braziliensis ........................................5

4. A child suffering from Visceral leishmaniasis caused by L.donovani ........................6

5. Different stages of Leishmania A. Promastigotes and B. Amastigotes .......................8

6. Life cycle of Leishmania .................................................................................8

7. Transition of procyclic promastigotes to metacyclic promastigotes inside

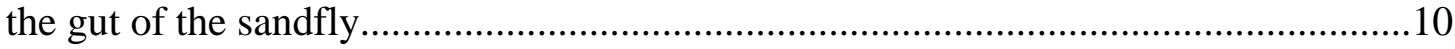

8. Drugs used in the treatment of leishmaniasis ....................................................12

9. Overview of Leishmania pathways involved in antimonial drug resistance ................19

10. Transmembrane proteins depicting single or multiple pass protein .........................24

11. A. Major intrinsic protein depicting aquaporin distribution and B. Difference between the pore size of human aquaporin (hAQP1)

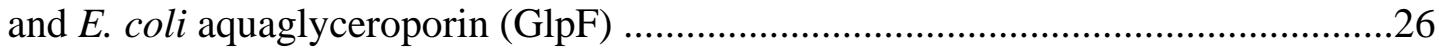

12. Expression of AQP1 in flagella of promastigote and contractile vacuoles in amastigotes

13. Topology of AQP1 depicting the extracellular C loop which differentiates between glycerol and metalloids 30 


\section{LIST OF FIGURES}

FIGURE

PAGE

14. Process of mRNA maturation by trans-splicing and polyadenylation in Leishmania 33

15. Mechanism of trans-splicing .35

16. Overview of the QuikChange site directed mutagenesis method (Agilent).

17. Principle of Regulatory Volume Decrease (RVD) phenomenon .64

18. Morphological changes in LdBOB promastigotes upon treatment with MG132

19. AQP1 is subjected to proteasome-mediated degradation ......................................73

20. Predicted topology of L. major AQP1 .......................................................... 74

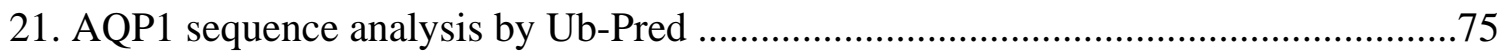

22. Protein sequence alignment of Leishmania AQP1 from six different species ...........76

23. N-terminus of AQP1 is disordered. AQP1 sequence analysis by A. PrDOS and

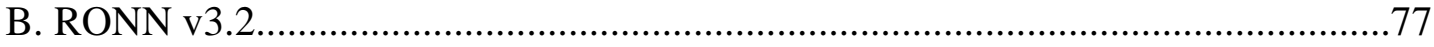

24. Volume regulation in LdBob transfectants in test strains ...................................79

25. Volume regulation in LdBob transfectants in control strains................................80

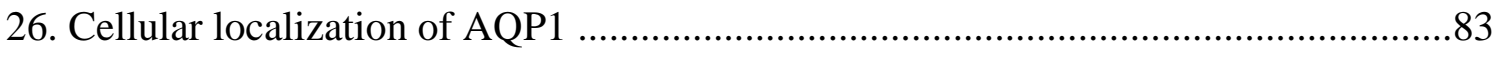

27. Immunofluorescence microscopy of LdBob transfectants ....................................85

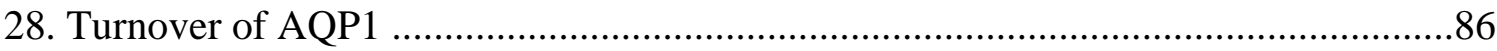

29. Proposed mechanism of AQP1 regulation at the post-translational level by probable ubiquitination and MPK2 intervention

30. Model of Pentostam action in macrophage associated amastigotes of Leishmania 100

31. 3’UTR sequence of AQP1 from L. donovani 


\section{LIST OF FIGURES}

FIGURES

PAGE

32. Sb(III) accumulation of promastigotes of different species of Leishmania

33. Efflux of Sb-trypanothione complex in plasma membrane vesicles isolated from different species of Leishmania.

34. Volume regulation of promastigotes of different species of Leishmania under hypoosmotic shock

35. Stability of AQP1 mRNA in promastigotes of different species of Leishmania......114

36. Exploring the role of 3'UTR in regulation of AQP1 using luciferase reporter system .....

37. Western Blot analysis of transfected promastigotes

38. Effect of AQP1 3'UTR from different species of Leishmania on luciferase (LUC) activity 118

39. Secondary structure prediction of AQP1 3'-UTR

40. Secondary structure prediction of AQP1 3'-UTR

41. Metalloid transporters in prokaryotes and eukaryotes 131 


\section{LIST OF ABREVIATIONS AND ACRONYMS}

A

Alanine

$\mathrm{ABC}$

ATP-binding cassette

ALP

.Alkyl-lysophospholipids

AQP

Aquaglyceroporin

ARE

AU-rich region

$\arg / \mathrm{R}$

aromatic arginine region

As(III)

Arsenite

As(V)

Arsenate

ATCC

American type culture collection

ATL

American Tegumentary leishmaniasis

ATP

Adenosine Triphosphate

AUBP $. \mathrm{A}+\mathrm{U}$ binding proteins

BHI Brain heart infusion media

BRF1 Butyrate response factor

BSA ..Bovine serum albumin

BSO .L-buthionine -(SR)-sulphoxime cDNA .Complementary DNA

CHX. Cycloheximide

CL .Cutaneous leishmaniasis CURE .CU-rich regions D Aspartic acid DAPI 4',6-diamidino-2-phenylindole DFMO DL- $\alpha$-difluor-o-methylornithine 
DGC Directional gene cloning

DHA Dihydroxyacetone

DNA Deoxyribonucleic acid dsDNA Double stranded DNA

$\mathrm{EC}_{50}$ Effective concentration

EDTA Ethylene diamine tetra acetic acid ERK Extracellular-signal regulated kinase

G Glutamate

GAPDH .Glyceraldehyde 3-phosphate dehydrogenase

GC Guanine cytosine GCS ..Glutamyl cysteine synthetase GSH Glutathione GST Glutathione S- transferase HEPES 4-(2-hydroxyethyl)-1-piperazinethanesulfonic acid HIV .Human Immunodeficiency Virus hnRNP .heterogeneous nuclear ribonucleoprotein HSP .Heat shock protein Hygro .Hygromycin B ICP-MS .Inductively coupled plasma mass spectrometry IgG ..Immunoglobulin G $\operatorname{Ig} M$ Immunoglobulin M IR Intergenic region IRES Internal ribosome entry sites K .Lysine 
KSRP KH-type splicing regulatory protein

Lb Leishmania braziliensis

LCTAS Leishmania conserved telomere-associated sequences

Ld Leishmania donovani

$\mathrm{Li}$ Leishmania infantum

Lm Leishmania major

LmACR2 Leishmanial As(V) reductase

Lp Leishmania panamensis

Lt Leishmania tropica

LUC .Luciferase $\mathrm{m}^{7} \mathrm{G}$ 7-methylguanosine MAPKK Mitogen-activated protein kinase kinase MCL Mucocutaneous leishmaniasis MIP Major Intrinsic Protein mOsm Milliosmolar MPK2 .Mitogen Activated Protein Kinase 2 MRP Multi-drug resistance related protein NEO Neomycin

ODC .. Ornithine decarboxylase

ORF .Open reading frame PAGE .Polyacrylamide gel electrophoresis PCD .Programmed cell death Pgp P-glycoprotein PI3K Phosphoinositide 3-kinase 
PKC Protein kinase C

PKDL Post Kala-azar leishmaniasis

PrDOS Protein disorder prediction system PSG Parasite secretory plug $\mathrm{R}$ Arginine

RACE .Rapid amplification of cDNA ends RBP RNA binding proteins RNAP RNA polymerase

RRM. RNA recognition motif

RVD Regulatory volume decrease

$\mathrm{Sb}(\mathrm{V})$ .Pentavalent antimony $\mathrm{Sb}(\mathrm{III})$ .Trivalent antimony ScAcr2p Saccharomyces cerevisiae arsenate reductase SDS Sodium dodecyl sulphate SE Standard error SIDER Small interspersed degenerate retroposons

SL Spliced leader RNA SSR Strand switch regions STR Short tandem repeats

$\mathrm{T}$ Threonine TDR Thiol-dependent reductase TR Trypanothione reductase TSH ..Trypanothione UTR .Untranslated region 
VL

..Visceral leishmaniasis

WHO .

..World Health Organization 


\section{CHAPTER 1}

\section{INTRODUCTION}

This chapter has been partially published:

Mandal, G., Govindarajan, V., Sharma, M., Bhattacharjee, H., and Mukhopadhyay, R.

Drug resistance in Leishmania (2014). In. Antimicrobial drug resistance: Principles and Practice for the Clinic and Bench. Eds, M. Ouellette and S. Lerner, Springer.

Mandal, G., Orta, J.F., Sharma, M. and Mukhopadhyay, R. Trypanosomatid aquaporins: Role in physiology and drug response (2014), Diseases. Vol 2, 3-23. 


\section{Introduction to leishmaniasis}

Leishmaniasis is a range of different diseases caused by an obligate intracellular protozoan parasite of the genus Leishmania (Order Kinetoplastida). It is classified as one of the most dreadful and neglected tropical diseases. Of the 30 species, 21 are known to infect mammals, including human beings. Leishmaniasis is one of the most commonly acquired cutaneous travel diseases, along with larva migrans, bacterial ulcers, and ecthyma (Von Stebut 2014). The disease is endemic in 88 countries, predominantly in the tropics, subtropics, and southern Europe. According to the World Health Organization (WHO), 90\% of the disease is concentrated in six "Old World" countries (Afghanistan, Algeria, Saudi Arabia, Iran, Sudan, and Syria) and in two "New World" countries (Brazil and Peru) (Figure 1) (Von Stebut, 2014).

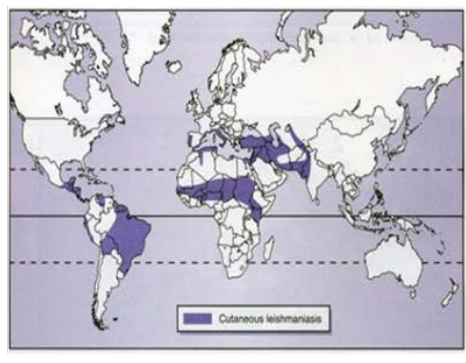

Cutaneous leishmaniasis

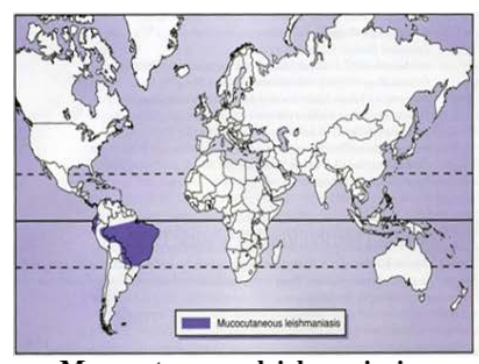

Mucocutaneous leishmaniasis

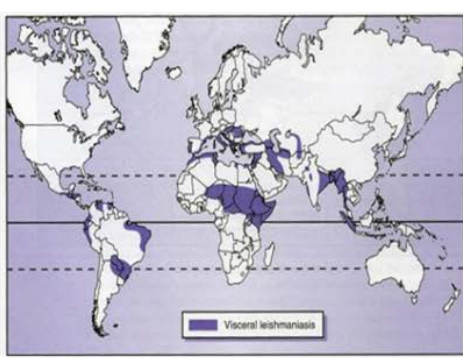

Visceral leishmaniasis

Figure 1. Geographical distribution of different types of leishmaniasis. Cutaneous leishmaniasis (CL), Mucocutaneous leishmaniasis (MCL) and Visceral leishmaniasis (VL). (http://www.stanford.edu/class/humbio153/InteractiveMaps/index.html)

Two million new cases are reported each year, adding up to almost 12 million people currently infected worldwide. Of great concern is a WHO report states that 350 million people are at a risk of infection (Reithinger et al., 2007). Leishmaniasis can be expressed in three different clinical forms: cutaneous (CL), mucocutaneous (MCL) and visceral leishmaniasis (VL). 


\subsection{Cutaneous leishmaniasis (CL)}

Cutaneous leishmaniasis (CL), also known as oriental sore, tropical sore, or chiclero ulcer, is the most common form of leishmaniasis in humans (Choi and Lerner, 2001). It is considered to be the least severe clinical form of the disease and causes skin sores or lesions usually at the site of the sandfly bite or in close proximity to it. The growth of intracellular amastigotes leads to localized lesions because of the release of cytokines at the inoculation site. In immunocompetent individuals, lesions heal after 618 months (Lesho et al., 2004). Cutaneous leishmaniasis is endemic in more than 70 countries; 90\% of these cases occur in Afghanistan, Algeria, Brazil, Pakistan, Peru, Saudi Arabia, and Syria (Reithinger et al., 2007) (Figure 1) The disease has an estimated annual incidence rate of 1.5- 2 million cases. In the Old World, L. major and L. tropica cause CL, whereas in the New World, L. mexicana and members of Leishmania (Viannia) braziliensis, Leishmania (Viannia) guyanensis, and Leishmania (Viannia) panamensis are the most common causes of the disease (Lesho et al., 2004). There are seven clinical features that correlate well closely with the diagnosis of Old World Cutaneous leishmaniasis: (i) Exposed site location, (ii) Pairing or clustering of lesions, (iii) Orientation of the skin sore along the skin crease, (iv) Volcano-like ulcer, (v) Inflammatory satellite papules, (vi) Proximal, sporotrichoid, subcutaneous nodules, and (vii) Subcutaneous induration extending underneath the skin lesion (Lesho et al., 2004).

Cutaneous leishmaniasis has four distinct forms as localized, disseminated, recidivans, and diffused leishmaniasis (Lesho et al., 2004). Localized Cutaneous leishmaniasis, also known as Baghdad boil, is usually the result of the development of inflammatory skin sores, leaving scars during the healing process. Usually, localized 
Cutaneous leishmaniasis is asymptomatic at first and later presents non-specific erythematous papules that can be easily confused with an insect bite. As time progresses, these erythematous papules expand slowly. The lesions appear to be nodular, plaque like, or ulcerative (Herwaldt, 1999) (Figure 2A and B). The speed of recovery from localized CL depends on the type of infective species.
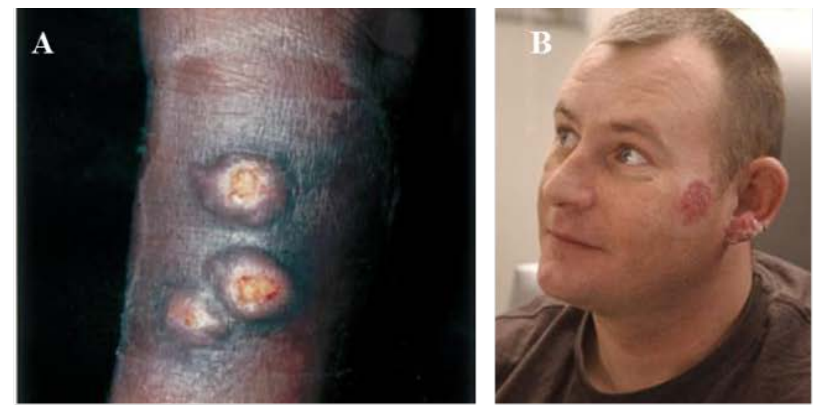

Figure 2. Cutaneous form of leishmaniasis depicting (A) localized cutaneous lesions (B) ulcerated lesion (Herwaldt, 1999)

An unusual variant of Cutaneous leishmaniasis is Leishmaniasis recidivans, caused by L. tropica, and often referred to as "Chronic Lupoid Leishmaniasis" because of its resemblance to lupus vulgaris. This is a chronic, non-healing or relapsing lesion that appears on or at the edge of the sores produced by previous cutaneous lesions. The onset of disease is usually within two years of the original disease, but much later onset has been reported following trauma or topical steroid use (Lesho et al., 2004).

Another rare form of the disease is diffused Cutaneous leishmaniasis, which is caused mainly by L. aethiopica in Africa and L. mexicana amazonensis in the New World. In these cases, the lesions are spread from one site to another. The infection is characterized by abundant parasitization and absence of an inflammatory response. 


\subsection{Mucocutaneous leishmaniasis (MCL)}

Mucocutaneous leishmaniasis (MCL) is one of the atypical forms of American Tegumentary leishmaniasis (ATL). This form of disease is endemic throughout areas of Latin America (Figure 1). Throughout the world, 12 million cases of MCL have been reported with an increase of 1.5 million new cases each year (Amato et al., 2008). L. panamensis, L. guyanensis, L. amazonensis are responsible for the clinical form of the disease, although it is most commonly caused by L. braziliensis. The infection caused by L. braziliensis leads to one of the most life-threatening and disfiguring forms of the disease, which is known as Espundia (Figure 3). Onset of MCL occurs months or years

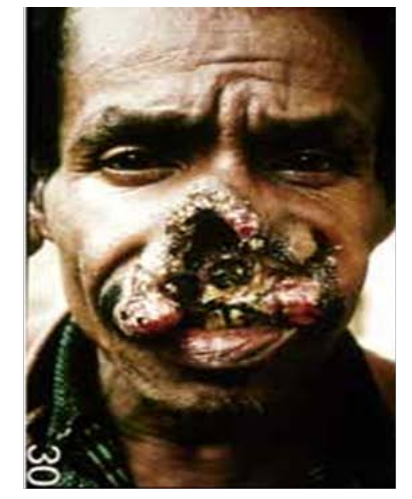

Figure 3. Patient suffering from Espundia caused by L. braziliensis (www.scientificearthconscientious6.wordpress.com)

after the primary cutaneous lesions appear. In this clinical form of leishmaniasis, parasites metastasize to mucous tissues through lymphatic or hematogenous dissemination. The MCL symptoms begin with nasal inflammation and stuffiness leading to later ulceration in the host's nasal mucosa. These hosts present concomitant lesions in the palate, larynx, and trachea. Mucocutaneous leishmaniasis is very difficult to treat, and if left untreated, potentially fatal secondary bacterial infections occur. Long- 
term consequences may also include severe facial disfiguration and respiratory disturbances that do not self-heal (Amato et al., 2008).

\subsection{Visceral leishmaniasis}

Visceral leishmaniasis (VL), most commonly known as Kala-azar is the most severe clinical form of the disease. According to a report by (Alvar et al., 2008) the incidence of VL is calculated as high as 0.2-0.4 million per year, with more than $90 \%$ in India, Bangladesh, Sudan, Ethiopia, and Brazil; mortality is estimated at $10-20 \%$, especially in poor regions. Visceral leishmaniasis is hypoendemic in Mediterranean countries (Savoia, 2015) (Figure 1), comprising 5\%-6\% of the reported global cases. Usually, infections remain asymptomatic, but conditions such as malnutrition and immune suppression related to HIV, may lead to the clinical symptoms associated with VL. Leishmania donovani and Leishmania infantum are the two major species

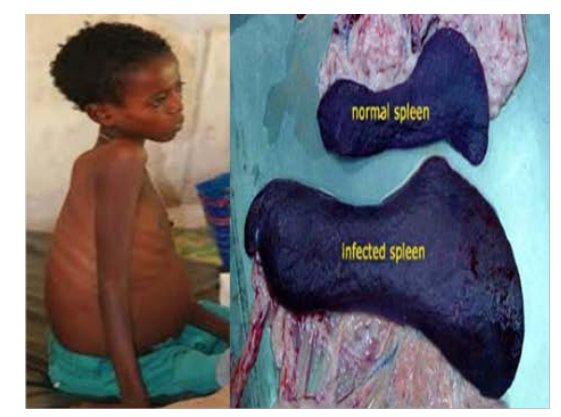

Figure 4. A child suffering from Visceral leishmaniasis caused by L.donovani. (www.scientificearthconscientious6.wordpress.com)

responsible for VL. Leishmania replicates in macrophages and mainly affects organs such as the liver, spleen and bone marrow (Alvar et al., 2008). Symptoms may take months or even years to develop (Monge-Maillo et al., 2014). Fatal if untreated, VL is 
associated with fever, weight loss, hepatosplenomegaly (Figure 4), anemia, leukopenia and thrombocytopenia (Monge-Maillo et al., 2014). Primary lesions in VL are rarely evident; they consist of small erythematous papules, which are known as "leishmaniomas". During the active stages of VL, the skin blackens, hence it is known as "Kala-azar” or "black fever" (Choi and Lerner, 2001). Patients suffering from VL have increased IgG and IgM levels, which leads to a depression in cell-mediated immunity. Depress immunity in turn then leads to patients becoming more susceptible to a variety of secondary opportunistic infections (Haldar et al., 1983). Immunodeficient individuals are unable to have successful treatment results leading to relapse later on (Berman et al., 1985).

Post kala- azar dermal leishmaniasis (PKDL) is caused by L. donovani and is endemic in East Africa and India. Twenty percent of patients in India recovering from visceral leishmaniasis contract PKDL (Bittencourt et al., 2002). Usually, small hypopigmented macules occur and then enlarge to form irregular patches. Lesions appear, and erythematous macules develop, particularly in the hypo-pigmented areas on the face. These macules are replaced by soft, painless, non-ulcerative, yellowish-pink nodules, which commonly appear on the face, earlobes, trunk, and genitalia. However, these nonulcerative nodules are less frequent in areas such as the hands and feet. Patients suffering from PKDL require longer courses of systemic medication (Choi and Lerner, 2001).

\subsection{Life cycle of Leishmania}

The life cycle of Leishmania includes two morphologically distinct forms, the promastigote and the amastigote stages. Promastigotes (Figure 5A) are slender spindle- 
shaped bodies with elongated anterior flagella that reside within the gut of sandflies. In contrast, amastigotes (Figure 5B) are small, oval-shaped, non-flagellated structures that
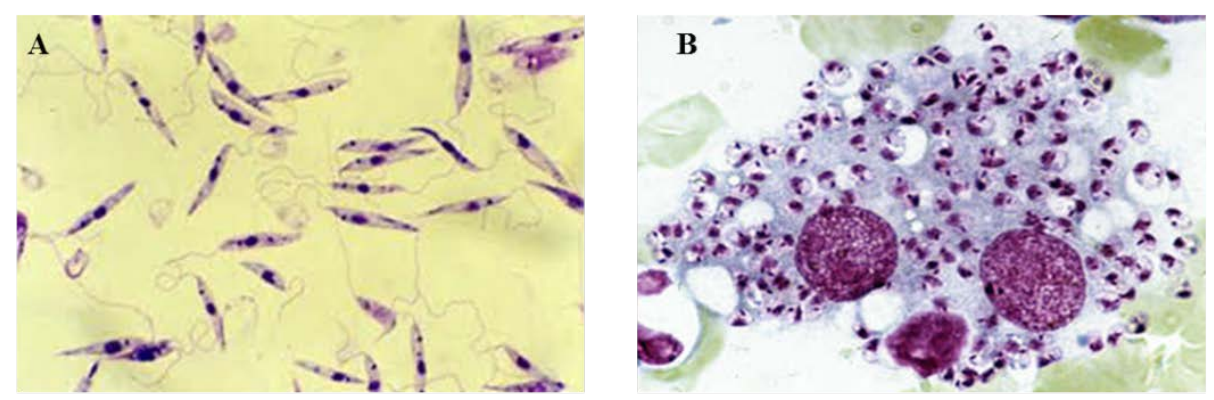

Figure 5. Different stages of Leishmania A. Promastigotes and B. Amastigotes reside inside the phagolysosomes of the host macrophages (Kamhawi, 2006). Amastigotes multiply by binary fission and finally burst open out of the infected cell. They are then taken up by non-infected macrophages or other mononuclear phagocytic cells, thus propagating the disease (Figure 6).

The pathogenicity cycle begins with an infected sandfly biting a vertebrate host. From the sandfly proboscis, the infected promastigote then gets injected into the skin of

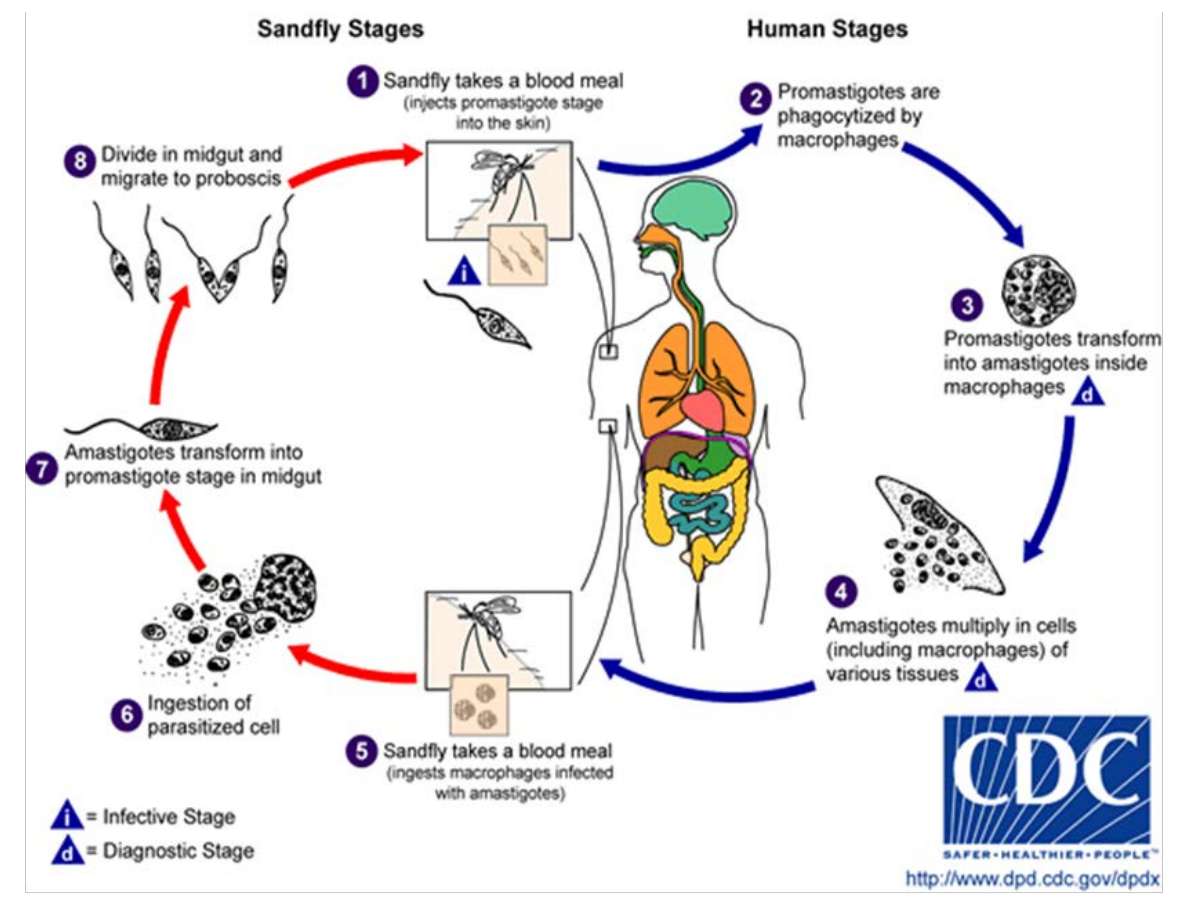

Figure 6 . Life cycle of Leishmania (www.cdc.gov/dpdx) 
the healthy host. Once the injected promastigotes are phagocytized by the host macrophages, they are transformed into amastigotes, which is known as the parasitic tissue stage (Figure 6). The rapid morphological transformation and parasitic development takes place because of differences in conditions such as $\mathrm{pH}$ and temperature inside the gut of the sandfly and the vertebrate host. Initially, amastigotes transform into small, sluggish, motile, dividing forms with short anterior flagellum known as procyclic promastigotes, which are located within the peritrophic matrix (Kamhawi, 2006) and observed in early bloodmeal. Procyclic form of promastigotes is relatively resistant to digestive enzymes (Kamhawi, 2006). After 48-72 hours, procyclics differentiate into nectomonad promastigotes, which are large, slender in shape, and non- dividing but highly motile. After they escape from the peritrophic matrix, the nectomonads attach themselves to the epithelial cell lining of the midgut and migrate towards the anterior thoracic midgut. The nectomonads then differentiate into leptomonads. Division of leptomonads results into a severe infection at the anterior midgut. Leptomonads then differentiate into non-motile haptomonads promastigotes with short flagella and highly motile metacyclic promastigotes with long anterior flagellum (Kamhawi, 2006). The non- dividing metacyclics usually accumulate at the stomodeal valve, ensuring the availability of the parasites for the transmission (Figure 7). To prevent or reduce the influx of blood into the midgut, leptomonads form a parasite secretory gel (PSG) plug. When the PSG plug and non-functional stomodeal valve are combined, a mechanism is created that enables the sandfly to expel or regurgitate the metacyclics located in front of or within the PSG plug onto the skin of the vertebrate host, restarting the life cycle (Ramalho-Ortigão et al., 2005). 


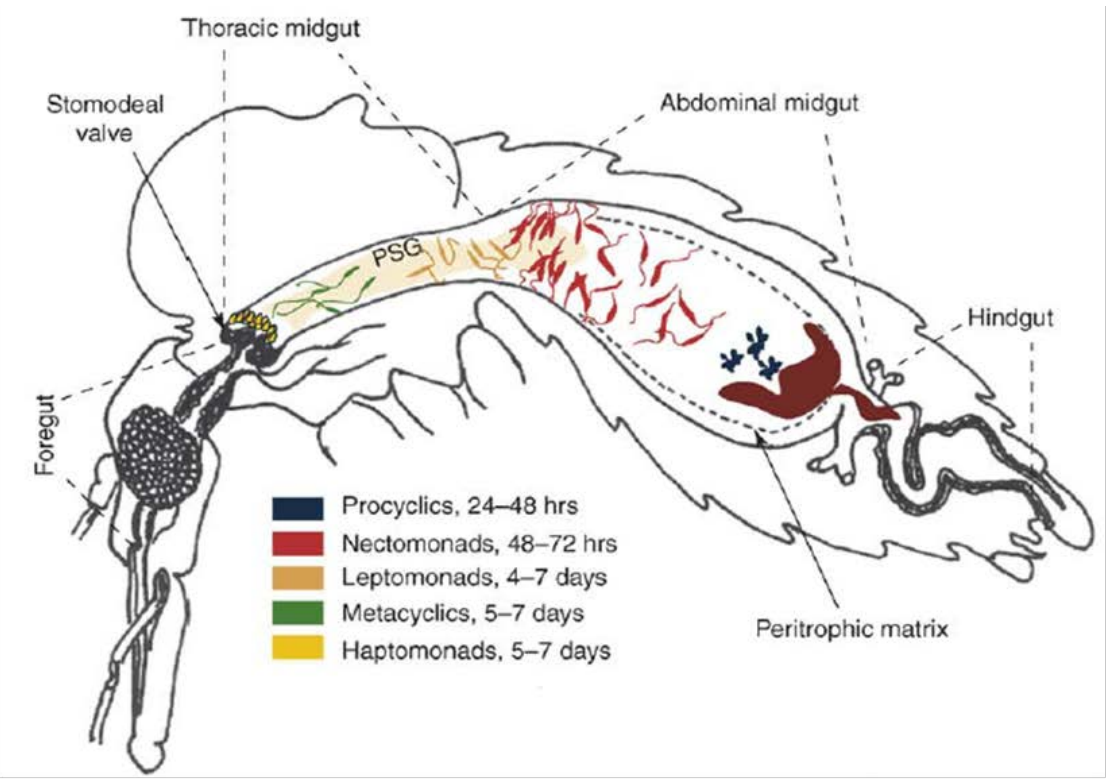

Figure 7. Transition of procyclic promastigotes to metacyclic promastigotes inside the gut of the sandfly (Kamhawi, 2006)

\subsection{History of leishmaniasis}

Leishmaniasis is an ancient disease tracing its history back to the Pre-Cambrian Era. Archaeological findings suggest the presence of chronic ulcers in mummified remains (Tuon et al., 2008). Any evidence confirming the presence of VL is absent from ancient records. Alexander Russel produced the first modern illustrations of the disease in 1756. In 1903, L.H. Donovan and W.B. Leishman clinically demonstrated the causative parasite in the splenic tissue, laying the cornerstone for widespread research of the disease. Momen and Cupolillo (2000) suggested that following the extinction of dinosaurs, the genus of Leishmania first appeared during the Paleogene of Paleocene. During the Paleocene period, Leishmania was divided into Sauro Leishmania, which affects reptiles, and the current Leishmania, which affects mammals. Studies offer vague 
yet logical theories about the origin of Leishmania (Tuon et al., 2008). Leishmania belongs to the phylum Kinetoplastida, which unfortunately has no current fossil record. Dooijes et al. (2000) suggested that the kinetoplastids are probably related to the euglenids, which according to Roger and Hug (2006), are related to the eukaryote super group Excavata, and hence, must have appeared during Ordovcian. Croan et al. (1997) reported that approximately 21 species of the genus Leishmania caused leishmaniasis. According to Hide et al. (2007), the species were divided into subgenera: Leishmania of the Old World and Viannia in the New World. The understanding of New World Leishmania comes from the appearance of mucosa lesions, which were common for preColombian inhabitants. The historical dispersal of the Leishmania parasite is based on the simultaneous origin of winged insects, which later created the lineage of the sandflies. The disintegration of the Pangea forming the Gondwana led to the division of two genera - Phlebotomus and Lutzomiya. Lutzomiya, according to Killick-Kendrick (1990), is responsible for the transmission of leishmaniasis in the New World. The vector-parasitehost transmission of Leishmania is the most plausible theory. During the Jurassic times, Gondwana was separated from Pangea, disseminating the vector (sandflies) and the hosts (mammals) throughout the world, with the exception of the Neoartic region, where it arrived during the Paleocene Era. It is believed that a primitive rodent brought Leishmania to the New World during the Neocene Era (Noyes, 1998). Noyes et al. (1997) are of the view that the parasite spread from North America to Asia, Europe and Africa via this route. Another theory suggests that migration from the Neotropics to the Neoartic and further into the Paleoartic might have been a possible route for the parasite (Yurchenko et al., 2006; Lukes et al., 2007) 


\subsection{Treatment}

Since the 1940s, Pentostam and Glucantime - the two organic pentavalent antimonial compounds have been the basis for all forms of treatment of leishmaniasis. However, pentavalent antimonial resistance to all clinical forms of leishmaniasis represents a challenge to the community of scientists and clinicians committed to effective treatment of the disease. In North-East India, more than $50 \%$ of patients suffering from VL have become resistant to Pentostam (Sundar and Rai, 2002), and there are several reports of trivalent antimony resistance (Ouellette et al., 1998). Amphotericin $\mathrm{B}$ and pentamidine are the second line of anti-leishmanial drugs (Figure 8). Alkyl

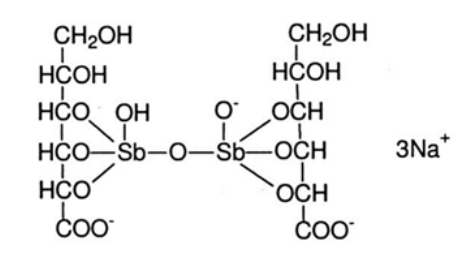

Sodium stibogluconate (Pentostam)<smiles>CCN(CC)CCCCCCNc1cc(OC)cc2c(C)ccnc12</smiles>

Sitamaquine (WR6026)<smiles>N=C(N)c1ccc(OCCCCCOc2ccc(C(=N)N)cc2)cc1</smiles>

4,4'-Diamidino- $\alpha, \omega$-diphenoxypentane di-isethionate (Pentamidine)
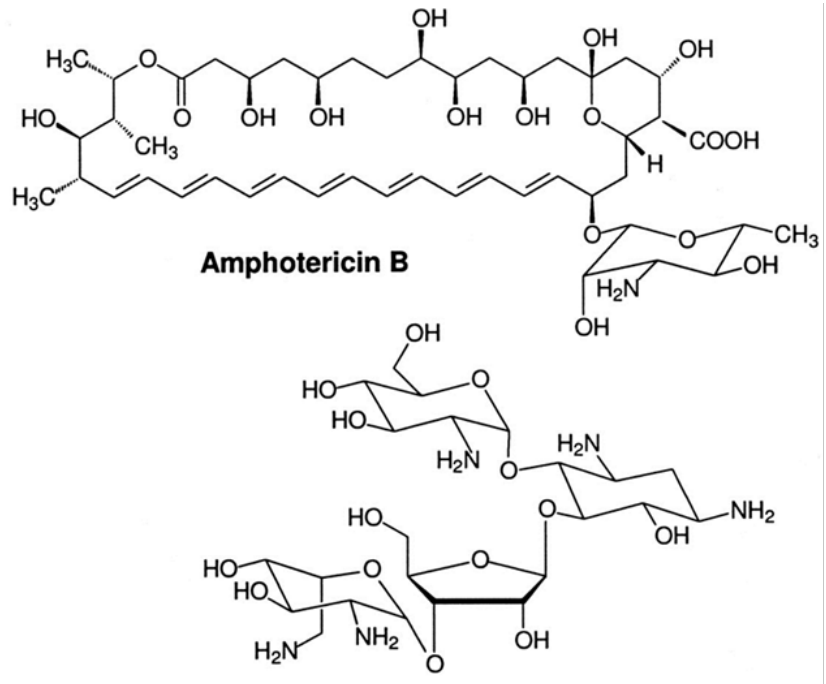

Paromomycin sulphate (Aminosidine)<smiles>CCCOP(=O)([O-])OCC[N+](C)(C)C</smiles>

Hexadecylphosphorylcholine (Miltefosine)

Figure 8. Drugs used in the treatment of leishmaniasis (Simon L. Croft et al.,2006) lysophospholipids (ALP), such as miltefosine (Figure 8) and edelfosine, used as anticancer treatment previously, have shown significant promise by counteracting the proliferative activity against Leishmania (Urbina, 1997). Miltefosine was the first oral 
drug given in India to treat cases of VL and antimony-resistance (Sundar and Rai, 2002). Other drugs, such as allopurinol, atovaquone, fluconazole, paromomycin, and sitamaquine (Figure 8), are currently in various stages of clinical trials (Sundar et al., 2011).

Both clinical and laboratory-induced drug resistance have been observed with these drugs. Understanding the drug resistance mechanism will help to identify drug targets and design tools to aid recognition of resistance early in the treatment process, enabling clinicians to begin alternate or combination therapies (Mandal et al., 2014). Leishmania employs several mechanisms to confer drug resistance (Borst and Ouellette, 1995). These include (i) downregulation of the uptake system(s) for the drug, (ii) intracellular sequestration, (iii) drug inactivation or modification, (iv) modification of the

drug target to prevent binding of the drug or overproduction of the target so that drug concentration becomes limiting, (v) more efficient repair of drug damage, and (vi) bypassing a blocked target (Mandal et al., 2014). My next section will concentrate on antimonial resistance in the next sections as my graduate research involves the regulation of the only antimonial uptake system in Leishmania, which is also involved in drug resistance.

\subsection{Antimonial mechanism of action and resistance}

Pentavalent antimonials have been used as the first line of treatment against all forms of leishmaniasis. The recommended dosage of pentavalent antimony [ $\mathrm{Sb}(\mathrm{V})]$ is $20 \mathrm{mg} / \mathrm{kg}$ for 20- 28 days. Longer courses of medication can induce toxicity in patients 
and present symptoms such as fatigue, body aches, electrocardiographic abnormalities, raised amino-transferase levels, and chemical pancreatitis (Herwaldt, 1999).

1.7.1 Mechanism of action: For the past six decades, cellular and molecular mechanisms have not been clearly understood for pentavalent antimonials. In vivo metabolic conversion of pentavalent $[\mathrm{Sb}(\mathrm{V})]$ to trivalent [Sb(III)], the active form of the drug was suggested half a century ago (Goodwin, 1995). When hamsters were infected with Leishmania garnhami and then treated with Glucantime $[\mathrm{Sb}(\mathrm{V})]$, it was observed that serum concentrations of $\mathrm{Sb}(\mathrm{III})$ and $\mathrm{Sb}(\mathrm{V})$ were similar; it was assumed that reduction of $\mathrm{Sb}(\mathrm{V})$ to $\mathrm{Sb}(\mathrm{III})$ could reduce the size and improve leishmanial ulcer healing (Lugo de Yarbuh, 1994). Several studies indicated that $\mathrm{Sb}(\mathrm{III})$ is more toxic than $\mathrm{Sb}(\mathrm{V})$ to either of the parasite stages. It was found that $\mathrm{Sb}(\mathrm{III})$ had a higher level of susceptibility as opposed to $\mathrm{Sb}(\mathrm{V})$ when amastigotes of $L$. infantum were grown under laboratory conditions (Sereno and Lemesre, 1997). Under the laboratory environment, amastigotes of $L$. infantum were also poorly responsive to meglumine $[\mathrm{Sb}(\mathrm{V})]$, as compared to amastigotes grown in human macrophages (Sereno et al., 1998). Sb(V) unresponsiveness suggested a putative reductase, which catalyzes the conversion of $\mathrm{Sb}(\mathrm{V})$ to $\mathrm{Sb}(\mathrm{III})$ within the macrophages. However, does Leishmania have a $\mathrm{Sb}(\mathrm{V})$ reductase? Given the fact that arsenical resistance Leishmania strains are mostly cross-resistant to antimonials, it was safely deduced that $\mathrm{Sb}(\mathrm{V})$ may be reduced by Leishmanial $\mathrm{As}(\mathrm{V})$ reductase as a result of arsenic and antimony being related metalloids. Zhou et al. (2004) used Saccharomyces cerevisiae arsenate reductase (ScAcr2p) sequence to identify and clone the $L$. major homologue, LmACR2. In terms of arsenate sensitivity, LmACR2, when overexpressed in Escherichia coli or S. cerevisiae arsenate reductase disrupted strains, complemented 
the $\operatorname{Sb}(\mathrm{V}) / \operatorname{As}(\mathrm{V})$ sensitivity. Pentostam sensitivity was found to be increased in intracellular amastigotes when $L$. infantum was transfected with LmACR2. Furthermore, when $\operatorname{LmACR} 2$ was purified, it was shown to reduce both $\mathrm{As}(\mathrm{V})$ and $\mathrm{Sb}(\mathrm{V})$ in vitro. As a result, it was theorized that $L m A C R 2$ was essential in reducing the pentavalent antimony in Pentostam to the Leishmania active trivalent form of the drug (Zhou et al., 2004).

Thiol dependent reductase (TDR1), a parasite-specific enzyme, was first identified and characterized by Denton et al. (2004) from L. major. Thiol dependent reductase 1 catalyzes the reduction of pentavalent form of antimonials to the trivalent form using glutathione as a reductant (Denton et al., 2004). The enzyme is a tetramer protein, containing two domain monomers, each of which is part of the omega class of glutathione S- transferases (GSTs). The enzyme is highly abundant in the mammalian stage of the parasite, which may explain the increased effectiveness of the drug during this stage (Sundar et al., 2007). Studies conducted by Berman et al. (1985) showed that L. mexicana promastigotes and amastigotes viability was impaired when exposed to 500 $\mu \mathrm{g} / \mathrm{ml}$ of sodium stibogluconate. Such exposure resulted in a decrease in the incorporation of radiolabel $\left[\left({ }^{14} \mathrm{C}\right.\right.$ leucine $),\left({ }^{3} \mathrm{H}\right.$ uridine $),\left({ }^{3} \mathrm{H}\right.$ thymidine $\left.)\right]$ into purine nucleoside triphosphate and an increase in the incorporation of radiolabel into purine nucleoside monophosphate, and diphosphate. Further studies suggested that antimonials possibly act by downregulating processes such as glycolysis and fatty acid $\beta$ - oxidation.

It was reported that one of the probable mechanisms of action may be the antimonials' ability to inhibit Leishmania topoisomerase I. Chakraborty et al. (1988) confirmed that $L$. donovani topoisomerase I is able to catalyze relaxation of supercoiled plasmid pBR322 aided by the inhibition of sodium stibogluconate. Different dosage 
inhibition of sodium stibogluconate suggested that antimonials do not interact with DNA as previously thought but with Leishmania topoisomerase I. Another possible mechanism may be the involvement of antimonials in the mitochondrial depolarization and associated thiol loss. As a result of the mitochondrial depolarization and the thiol loss, there is less production of ATP and an increase of oxidative bursts within the phagolysosomes of macrophages (Mehta and Shaha, 2006). The oxidative burst occurring within the macrophages takes place via the phosphorylation of phosphoinositide 3- kinase (PI3K), protein kinase C (PKC) Ras and extracellular-signal regulated kinase (ERK). Inhibition of these proteins emphasizes the importance of oxidative bursts within phagolysosomes of infected macrophages (Mookerjee Basu et al., 2006).

Programmed cell death (PCD) by antimony use is one of the mechanisms responsible for killing the parasites involving DNA fragmentation and externalization of phosphotidylserine on the outer surface of the membrane (Sereno et al., 2001). In vitro studies indicate that trypanothione reductase (TR) and glutathione synthetase are inhibited by $\mathrm{Sb}(\mathrm{III})$. Rapid efflux of trypanothione (TSH) and GSH occurs as soon as promastigotes and amastigotes are exposed to $\mathrm{Sb}(\mathrm{III})$, which sometimes compromises the redox potential of the cell. Studies have shown that $\mathrm{Sb}(\mathrm{III})$ is active against both stages of the parasite, whereas $\mathrm{Sb}(\mathrm{V})$ is active only in intracellular amastigotes. Shaked Mishan et al. (2001) showed that $\mathrm{Sb}(\mathrm{V})$ is reduced to $\mathrm{Sb}(\mathrm{III})$ in axenic amastigotes.

1.7.2 Mechanism of resistance: The mechanism of antimonial resistance is an important area of research in Leishmania. Several studies have indicated that antimonial resistance is multifactorial. Parasites use one or more mechanisms to become unresponsive to 
antimonials. Antimonial drugs must enter the host cell and act against the intracellular parasite. However, the antimonials $[\mathrm{Sb}(\mathrm{V})]$ route of entry of the parasite into the macrophage is not yet well understood, but as stated in an earlier section, $\mathrm{Sb}(\mathrm{V})$ is reduced to $\mathrm{Sb}$ (III) to become anti-leishmanial. Accumulation of $\mathrm{Sb}(\mathrm{III})$ has been observed in both stages of Leishmania, but variation in $\mathrm{Sb}(\mathrm{III})$ and $\mathrm{Sb}(\mathrm{V})$ accumulation levels suggests different routes of entry for the two forms of antimony. In an earlier section (1.7.1), we described how LmACR2 confers Pentostam hypersenitivity and displays arsenate and antimonate reductase activities. Leishmania major arsenate reductase (LmACR2) dephosphorylates phosphotyrosine residues in leishmanial proteins (Zhou et al., 2004). Thiol dependent reductase 1 is a reductase whose activity was found to be 10 times higher in L. major amastigotes as compared to promastigotes (Fakhri Jeddi, 2011), but the enzymatic function in antimonial resistance has yet to be determined in clinical isolates (Mandal et al., 2015). The most common factors for antimonial resistance are: (i) MRPA ; (ii) thiols ; (iii) an unknown efflux system and (iv) aquaglyceroporin (AQP1) (Mandal et al., 2015).

The ATP- binding cassette (ABC) transporters play an essential role in a variety of physiological processes. Their presence extends from prokaryotes to humans, and they are evolutionarily highly conserved (Manzano et al., 2013). The ABC transporters are involved in the transport of different compounds across biological membranes, including anti-leishmanicidal agents, such as antimonials, miltefosine, pentamidine and sitamaquine, using energy through ATP hydrolysis (Manzano et al., 2013). They have also been identified in Leishmania, in which they have been amplified extra- 
chromosomally in many species in response to a variety of drugs under controlled environments (Ouellette et al., 1998). There are two types of ABC transporters responsible for multi- drug resistance in mammalian cells: P-glycoprotein (P-gp) and multi-drug resistance- related protein (MRP). The MRP1 family is known to be involved in thiol- associated efflux and metal resistance (Haimeur et al., 2000). Analyses of the Leishmania genome lead to the identification of eight putative protein homologues belonging to the MRP1 family, which are functionally distinct from mammalian MRP. In Leishmania, one of the ABC transporters is PGPA (aka MRPA), which belongs to the ABCC subfamily (ABCC3) and plays a significant role in metal resistance, extrusion and sequestration of drug- thiol conjugates into a parasite vacuole. Its role in metal resistance was confirmed by several transfection studies in laboratory strains (Papadopoulou et al., 1994). 


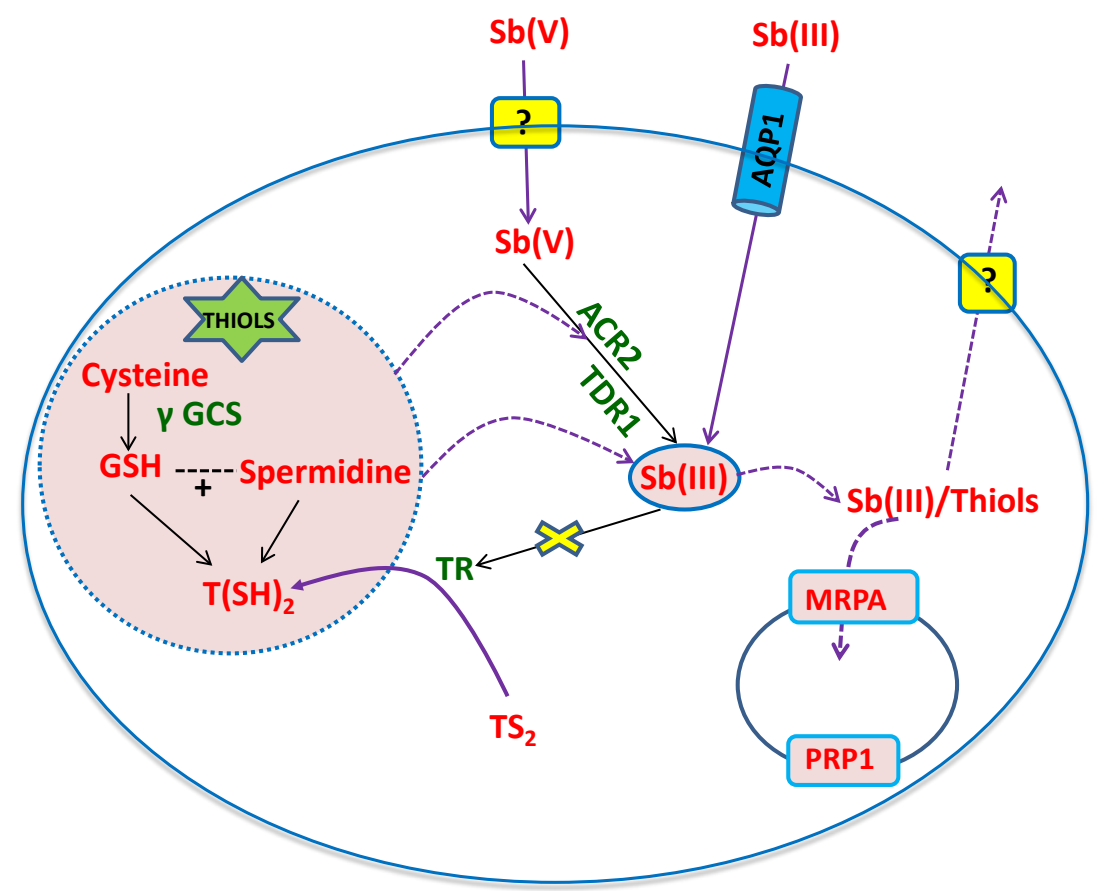

Figure 9. Overview of Leishmania pathways involved in antimonial drug resistance (Decuypere et al., 2012)

Mukhopadhyay et al. (1996) reported that arsenite/antimonite resistant $L$. tarentolae promastigotes overproduce trypanothione (TSH), a unique glutathionespermidine conjugate found only in trypanosomatids. Ornithine decarboxylase (ODC) and $\gamma$-glutamylcysteine synthetase $(\gamma$-GCS) are the two rate-limiting enzymes present in the polyamine and glutathione biosynthetic pathways, respectively (Haimeur et al., 1999). These enzymes facilitate trypanothione overproduction, but increased levels of TSH alone are not sufficient to generate metal resistance (Figure 9). 
Reversion of metal resistance can be achieved by downregulation of TSH levels by specific $\gamma$-GCS or ODC inhibitors, such as L-buthionine-(SR)-sulphoxime (BSO) and DL- $\alpha$-difluor-o-methylornithine (DFMO), respectively (Haimeur et al., 1999). Grondin et al. (1993) reported an increase in thiol levels as a result of an overexpression of either ODC or $\gamma$-GCS in L. tarentolae wild- type cells. In addition, the overexpression of these specific inhibitors was almost equivalent to resistant mutants with the difference that the cells did not show arsenite resistance. Whereas, when ODC or $\gamma$--GCS was cotransfected with MRPA in wild type cells, arsenite resistance was observed (Haimeur et al., 1999; 2000). The acquired resistance was reversed when transfectants were treated with BSO- a thiol depletory (El Fadili et al., 2005). These studies established that MRPA and increased TSH levels act synergistically. The limiting factor involved in both the drug transport conjugates and arsenite and/ or antimonite resistance was correlated to TSH availability (Ouellette et al., 1991). The MRPA is localized in small vesicles, near flagellar pockets, that sequester arsenic/antimony-thiol conjugates and are later disposed outside the cell (Legare et al., 2001).

Callahan et al. (1994) reported that overexpression of MRPA resulted in higher influx of antimony rather than higher efflux. The difference of influx to efflux found might be the result of an inhibitory effect through associated membrane protein interactions. This suggests that MRPA acts as an intracellular transporter rather than an efflux transporter and has a significant role in antimony resistance (Figure 9). Dey et al. (1994) reported that the transport experiments in arsenite resistant $L$. tarentolea mutants showed the presence of an active efflux system. However, the results did not correlate 
with PGPA amplification in L. tarentolae or resulted in any significant accumulation of arsenite even after transfection. On the other hand, L. major transfectants showed a decrease uptake of antimony which serves as support when attempting to explain its resistance. ABCC7-PRP1 is the second ABC transporter involved in antimony resistance. When the PRP1 gene is overexpressed in pentamidine-resistant Leishmania cell lines, it confers significant cross-resistance to antimony, but the localization and mechanism by which this protein confers resistance needs to be determined (Ashutosh et al., 2007). Other members of the ABCC subfamily, such as ABCC4, ABCC5 and ABCC7, also confer resistance to antimonials (Leprohon et al., 2009). Recently, a new ABC half-transporter ABCI4 was characterized in L. major. This transporter is localized both on the mitochondria and plasma membranes and is involved in efflux of Sb (III)thiol conjugate. Overexpression of ABCI4 is a clear indicator of L. major antimony resistance (Manzano et al., 2013). Therefore, ABCI4 can be considered a potential thiol$\mathrm{X}$ pump, which recognizes metal- conjugated thiols and confers antimony resistance in Leishmania (Manzano et al., 2013).

Several studies in Indian $L$. donovani clinical isolates indicated higher TSH levels, which protect parasites from Sb(III) induced oxidative burst. It was observed that thiol biosynthesis was much higher as compared to $\mathrm{Sb}$ (III) mediated thiol loss. Leishmania mexicana and Leishmania donovani were the first two parasites in which antimony transport studies were conducted in both the promastigotes and amastigotes stages using Pentostam $\left({ }^{125} \mathrm{Sb}\right)$. Inductively coupled plasma mass spectrometry (ICPMS) revealed the accumulation of two forms of antimony $(\mathrm{Sb}(\mathrm{V})$ and $\mathrm{Sb}(\mathrm{III}))$ in both 
stages of parasite. Nonetheless, accumulation of $\mathrm{Sb}(\mathrm{V})$ was found to be much more in axenic amastigotes in some species of Leishmania (Ashutosh et al., 2007). It is known that Pentostam consists of $\mathrm{Sb}(\mathrm{V})$ complexed to gluconate and gluconate inhibits the uptake of $\mathrm{Sb}(\mathrm{V})$ in amastigotes. Therefore, it was speculated that there should be some other mechanism for $\mathrm{Sb}(\mathrm{V})$ uptake inside the parasites. Unfortunately, neither $\mathrm{As}(\mathrm{V})$ nor phosphate was able to compete with the uptake of Pentostam, which ruled out the possibility that $\mathrm{Sb}(\mathrm{V})$ uses an $\mathrm{As}(\mathrm{V})$ transporter. Studies showed that $\mathrm{Sb}(\mathrm{III})$ and $\mathrm{As}(\mathrm{III})$ enter the cells via the same route, because the accumulation of $\mathrm{Sb}(\mathrm{III})$ is competitively inhibited by As(III), a related metal (Gourbal et al., 2004).

Gourbal et al. (2004) identified the first aquaglyceroporin or AQP1 in Leishmania. This discovery was a breakthrough in the field of antimony resistance. In Leishmania, to date, AQP1 is the only identified aquaporin which facilitates trivalent antimony $[\mathrm{Sb}(\mathrm{III})]$ transport. In both stages of the parasite, AQP1 modulates drug sensitivity when overexpressed. Several studies indicated that overexpression of AQP1 gene in L. major produces hypersensitivity to $\mathrm{Sb}(\mathrm{III})$, whereas gene deletion makes the parasite resistant (Mandal et al., 2010). After, the gene expression analysis of $L$. donovani field isolates from Nepal were completed, it was observed that the downregulation of AQP1 plays a major role in the reduced uptake of antimonite (Decuypere et al., 2005).

Leishmania major aquaglyceroporin 1 (AQP1) is the major focus of my research project, so I will be describing in detail in the following sections the importance of AQP1 in physiological roles and drug resistance in Leishmania. During the life cycle of 
Leishmania (described in section 1.4), the parasite faces many osmotic challenges during its transmission from vector (sandfly) to the mammalian host. In order to combat these challenges, the parasite needs to regulate water and solute movements. It is widely known that aquaporins, a special type of integral membrane proteins, facilitate the movement of water, and that they are ubiquitously present from bacteria to mammals. It has already been shown that AQP1 plays a major role in providing promastigote with an osmotic fitness, which is required for successful transmission and establishes infection in the mammalian host (Gourbal et al., 2004).

\subsection{Membrane proteins}

Membrane proteins are the proteins which interact with biological membranes. They are anchored in the phospholipid bilayer. Each phospholipid molecule has a hydrophilic head region and a hydrophobic tail region. Membrane proteins are responsible for a variety of functions, including movement of molecules and ions across the membrane, elicitation of numerous signaling pathways, and membrane enzymatic activities (Lodish, 2000). Singer and Nicolson,1972 classified these proteins into two classes: Peripheral and Integral membrane proteins.

\subsubsection{Peripheral membrane protein or extrinsic protein}

Peripheral membrane proteins are always facing the hydrophilic side of the phospholipid bilayer and do not interact with the hydrophobic core. They are attached to the membrane by hydrophobic, electrostatic or other non-covalent interactions. These proteins usually dissociate from the membranes when treated with solutions of high $\mathrm{pH}$ 
or high salt concentration, but these conditions do not disrupt the phospholipid bilayer (Lodish, 2000).

\subsubsection{Integral membrane proteins or intrinsic proteins}

Integral membrane proteins are permanently attached to the membrane. These proteins have hydrophobic side chains which interact with fatty acid acyl groups of the membrane phospholipids. Integral membrane proteins are usually transmembrane proteins. They extend out through the lipid bilayer so that one end is hydrophobic, in contact with the interior of the cell, while the other part is hydrophilic, exposed to the exterior of the cell. Single pass membrane proteins can cross the membrane only once, whereas multiple pass membrane proteins can cross membrane several times (Figure 10). Aquaporins and aquaglyceroporins are the examples for this class of protein (Lodish, 2000 Molecular Biology).

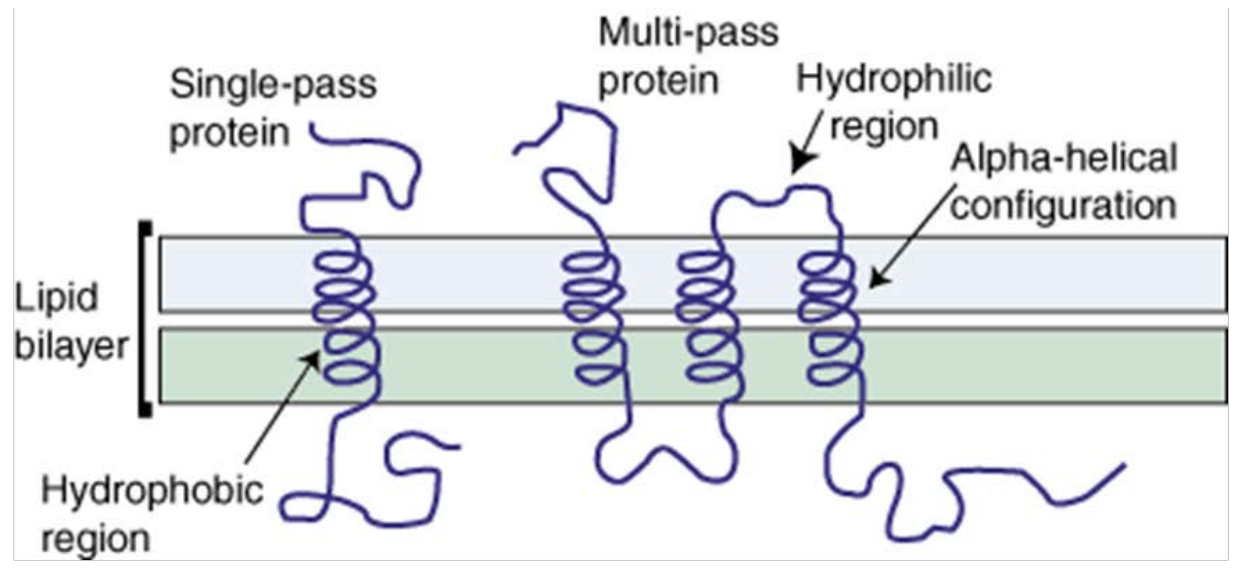

Figure 10. Transmembrane proteins depicting single or multiple pass protein (Lodish, 2000) 


\subsection{Parasite aquaporins}

Trypanosomes are unicellular and pathogenic parasites known to be one of the major causes of morbidity and mortality in tropical and subtropical regions worldwide. Leishmania species (leishmaniasis), Trypanosoma brucei (African sleeping sickness) and Trypansoma cruzi (Chagas disease) are the pathogens which belong to this group of parasites. Trypanosomatid parasites have digenetic life cycles. During transmission from the sandfly to the mammalian host, the parasite faces significant osmotic challenges. When the parasite resides inside the gut of the vector, it differentiates into infective forms and spreads the infection to specific organs. In contrast, within the mammalian host, parasites need to find appropriate environments to escape the host defense mechanism, facilitating the pathogenicity pathway. Osmotic signals are critical to cellular morphogenesis and migration in other organisms. All living cells need to regulate water and solute movements efficiently in order to respond to osmotic signals (Mandal et al., 2014). The lipid bilayer membranes present in living cells are impermeable to water and other polar solutes, hence the need of employing membrane transporter (s) that permit the rapid regulated movements of water and non-solutes in and out of the cell by extra- and/ or intracellular stimulus. In 1992, Peter Agre and his co-workers were the first to identify a channel- forming integral membrane protein, which facilitates water transport and is essential for all life processes. The members of this channel protein are ubiquitously present in all organisms and are collectively called aquaporins (AQP). Aquaporins (AQP) belong to the family of Major Intrinsic Proteins (MIP) that regulate the flow of water, glycerol and other uncharged solutes but are impermeable to charged particles and protons. Water molecules move across the membrane channel in a single file. AQPs are 
distributed widely ranging from E.coli to humans. Depending on the substrate specificity, the aquaporins family can be divided into two sub groups: "orthodox aquaporins" that conduct only water and "aquaglyceroporins" that conduct glycerol and other small uncharged molecules along with water (Engel and Stahlberg 2002; Hub and de Groot 2008) (Figure $11 \mathrm{~A}$ and B).
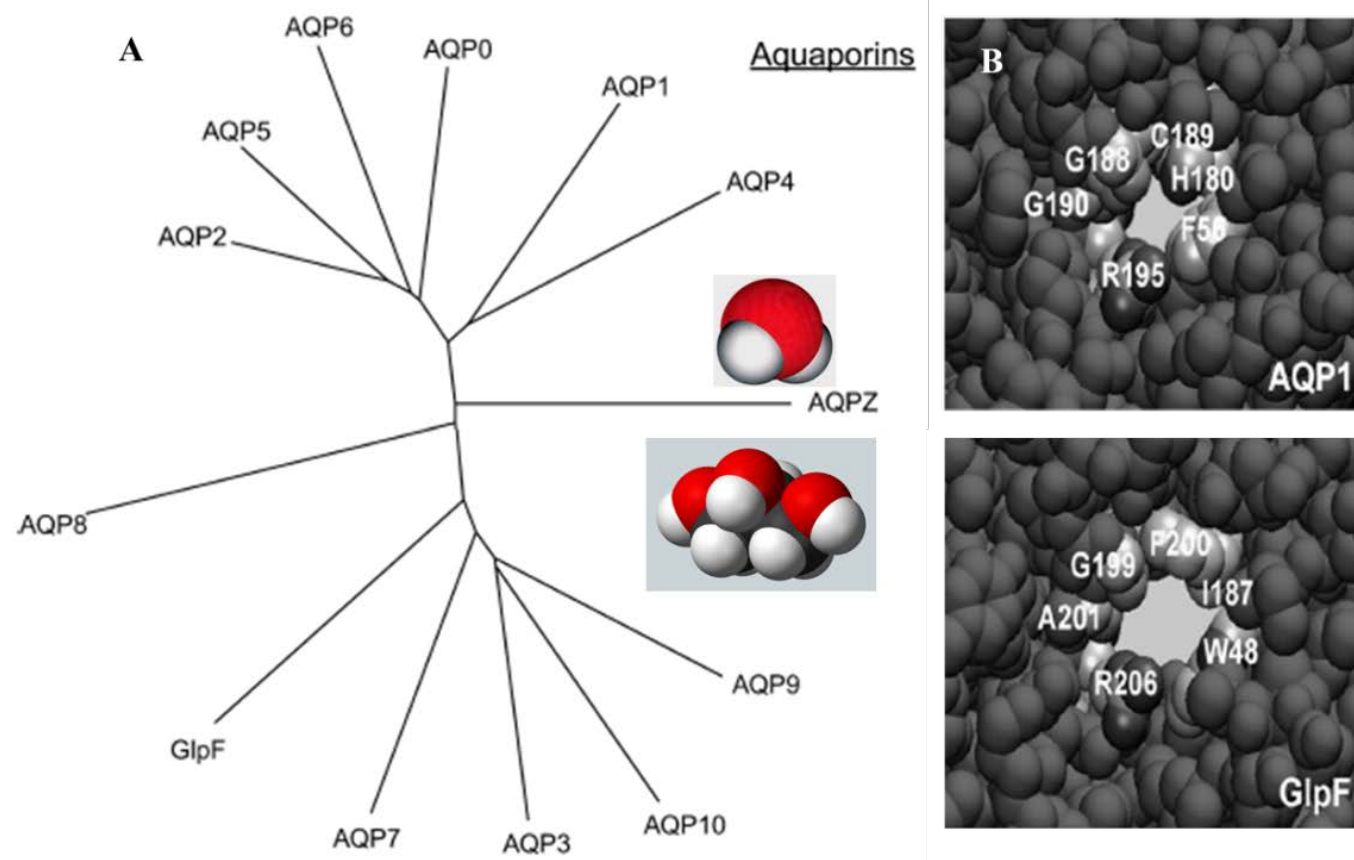

\section{Aquaglyceroporins}

Figure 11. A. Major intrinsic protein depicting aquaporin distribution and B. Difference between the pore size of human aquaporin (hAQP1) and E.coli aquaglyceroporin (GlpF) (Masato Yasui, J. 2004)

Members of the AQP superfamily are tetrameric proteins, and each monomer consists of six transmembrane helices and two short half helices which fold into the center of the channel (Jensen et al., 2002; Hub and de Groot 2008). Interestingly, each monomer functions independently, and the central pore of the structure is impermeable to any solute. The substrate specificity arises from the pore mouth size. It was found that 
the E.coli aquaglyceroporins GlpF pore mouth size is bigger than human orthodox aquaporin hAQP1 (Engel and Stahlberg, 2002). One of the constriction regions at the pore mouth is known as the aromatic arginine (arg/R) constriction, and the other is known as the Aspargine-Proline-Alanine (NPA) region, which is located at the middle of the pore (Beitz, 2006). The center of the pore is formed by two NPA motifs. These NPA motifs act as a proton filter, which functions as a cap for the amino acids at the positive ends of helices $\mathrm{B}$ and $\mathrm{E}$, and behave as hydrogen donors to the oxygen atoms of passing permeants (Mandal et al., 2014). In mammals, 13 AQPs (0-12) have been identified so far (Hara-Chikuma and Verkman 2006). Human AQPs play an important role in homeostasis of water, lung moistening, tears and saliva secretion, glycerol and fat metabolism. It also facilitates cell migration, neural signal transduction, cell volume regulation and organellar physiology. Several AQPs have been reported in many parasitic protozoa. Plasmodium falciparum has a single aquaporin, Trypanosoma brucei contains three, Trypanosoma cruzi contains four, and five have been reported in Leishmania (Beitz, 2005). Parasitic aquaglyceroporins are better water facilitators than bacterial GlpF, and they are responsible for the conduction of metalloids. The only characterized AQP1 in Leishmania is LmAQP1, which conducts water and glycerol almost at the same rate. LmAQP1 also transports glyceraldehydes, dihydroxyacetone and methyl glyoxyl (Figarella et al., 2007) and facilitates the uptake of metalloids such as arsenite and antimonite. 


\subsubsection{Role of AQP1 in Leishmania}

The L. major genome codes for five aquaporins: LmAQP1, LmAQP $\alpha, L m A Q P \beta$, LmAQP $\gamma$, and LmAQP $\delta$. Bacterial aquaporins are closely related to LmAQP1 in $L$. major, whereas the other L. major aquaporins (LmAQP $\alpha-\delta)$ share some properties with those of plant aquaporins (Song et al., 2014). LmAQP1 also has significant permeability for water. As described earlier, AQP1 is the sole reported Sb(III) facilitator. Amastigotes are more sensitive to $\mathrm{Sb}(\mathrm{III})$ than promastigotes, indicating that AQP1 may be differentially regulated in the two developmental stages of the parasite. Disruption of one of the two AQP1 alleles in Leishmania major resulted in a 10-fold increase in resistance to Sb(III) (Gourbal et al., 2004). Resistance to Sb(III) has been corroborated by downregulation of $A Q P 1$ mRNA levels in clinical isolates of drug- resistant $L$. donovani (Decuypere et al., 2005). On the basis of these studies, it can be hypothesized that the AQP1 present in the plasma membrane constitutes the rate-limiting step in the uptake of the activated form of the antimonial drugs. Overexpression of AQP1 makes the wild type parasites $>100$ fold more sensitive to Sb(III). More importantly, overexpression of AQP1 can abolish the drug- resistant phenotype in drug-resistant isolates. Therefore, AQP1 is instrumental in the successful treatment of leishmaniasis using antimonials. In contrast to Plasmodium and Trypanosome AQPs, which are inhibited by mercury, AQP1 is a mercurial-independent channel (Uzcategui et al., 2008; Mukhopadhyay et al., 2011). AQP1 also helps in the conduction of glycerol, methylglyoxal, glyceraldehydes, dihydroxyacetone (DHA), erythritol, and adonitol. Expression of LmAQP1 is limited to the flagellum of promastigotes; in amastigotes, it is 
found in the flagellar pocket, rudimentary flagellum and contractile vacuoles (Figarella et al., 2007) (Figure 12).

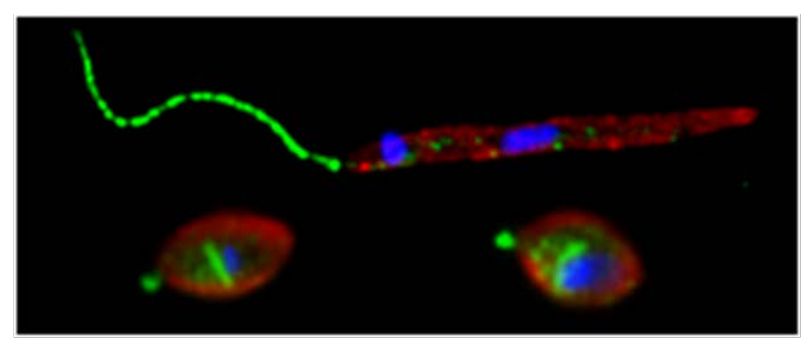

Figure 12. Expression of AQP1 in flagella of promastigote and contractile vacuoles in amastigotes (Figarella et al., 2007)

The presence of AQP1 in the flagella aids the parasite in volume regulation and osmotaxis, two important factors for overcoming the osmotic challenges the parasite faces during its life cycle. Therefore, it is evident that AQP1 is critical to Leishmania physiology and drug resistance. At physiological $\mathrm{pH}$, the trivalent metalloids behave as molecular mimics of glycerol and are conducted through AQP channels. It was reported that a single mutation in glutamate 152 (G152) of the extracellular C loop of AQP1 helps in the differentiation between glycerol and metalloids (Uzcategui et al., 2008) (Figure 13).

Mukhopadhyay et al., 2011 reported that alanine 163 in loop C is present near the pore mouth and is significantly important for the function of the channel, as the alteration of alanine leads to channel function inactivation. In the absence of transcriptional control, the Leishmania species primarily regulates its genes at the post-transcriptional and/or post-translational levels (Mandal et al., 2012). Mandal et al. (2012) reported that mitogen activated protein kinase 2 (MPK2) positively regulates AQP1 stability through phosphorylation of the threonine 197 (T197) residue. Aquaglyceroporin 1 (AQP1) 


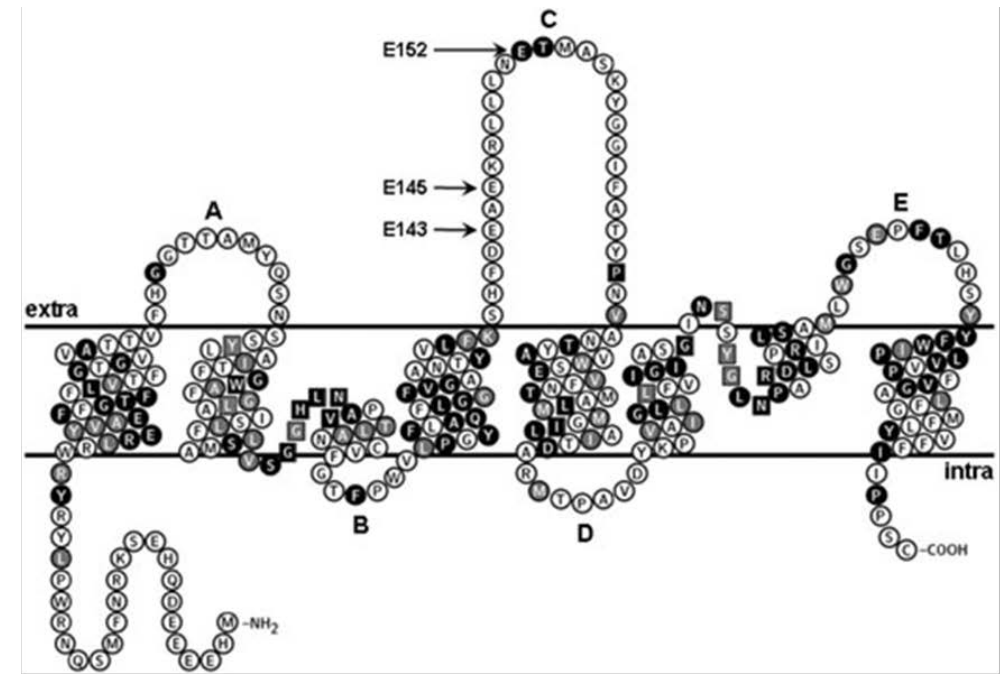

Figure 13. Topology of AQP1 depicting the extracellular $C$ loop which differentiates between glycerol and metalloids (Uzcategui et al., 2008)

molecules phosphorylated at T197 are more stable compared to molecules without phosphorylation at T197. Similarly, alteration of MPK2 destabilizes AQP1 because of the absence of phosphoryl transfer. Interestingly, phosphorylation at T197 also affects localization of AQP1 and alters drug sensitivity and osmoregulatory activity of the parasite. Evidence continues to suggest that AQP1 contributes significantly to the ability of the parasite to combat osmotic challenges during transmission from vector to host.

\subsection{Gene regulation in Leishmania}

Leishmania species have a haploid genome. It is organized into 36 chromosomes, which contains 911 RNA genes and 39 pseudo genes (Ivens et al., 2005). The Leishmania genome encompasses 8272 genes encoding proteins. The Old World and the New World Leishmania species have 36 and 34 or 35 chromosomes respectively (Wincker et al., 1996), which vary in size from 268 to 2,680 Kb (Uliana et al., 2007). The Leishmania genome is organized in the nucleus, which contains chromosomal and 
episomal DNA. The genome is also comprised of kinetoplast, which contains independent replicating DNA molecules. Leishmania is usually a diploid organism but in some cases, aneuploidy has also been observed. For the genome-sequencing project, $L$. major has been used as a reference strain, which possesses three chromosome 1 homologues. The genome size of $L$. major is $33.6 \mathrm{Mb}$ with an overall of $59.7 \%$ GC content (Ivens et al., 2005). In Leishmania, active retrotransposable elements are absent (Bringaud et al., 2006). Sub-telomeric regions have a majority of repeat elements (Wickstead et al., 2003). All Leishmania species contain a variety of hexameric repeat 5'-TAGGGT-3' and octameric repeat 5'-TGGTCATG-3' in telomeres. Both the hexameric repeats and octameric repeats are known as Leishmania conserved telomereassociated sequences (LCTAS) (Fu and Barker, 1998). In addition, Leishmania and Trypanosoma genomes are organized into long arrays of tandem coding sequences. These tandem coding sequences are positioned and transcribed into polycistronic primary transcripts (Ivens et al., 2005). The directional gene clusters (DGCs) are not sized uniformly, varying from a few kb to more than 1.2 Mb (Uliana et al., 1996). Sequences present between two divergent transcription units are called strand-switch regions (SSRs). The nucleotide sequences present in the strand-switch regions do not show a particular consensus sequence for any RNA polymerase known promoter (Tosato et al., 2001). In general, transcription in trypanosomes and Leishmania by RNA polymerase II is quite unusual and unique. RNA polymerase II promoters encode approximately 120 copies of the spliced leader RNA (SL RNA) gene (Gilinger and Bellofatto, 2001). These RNA polymerase II promoters are found in the intergenic sequences of the tandem array as mentioned above. In Leishmania, promoters for RNA polymerase I share similarities 
with other organisms such as: insensitivity to $\alpha$ - amanitin, poor sequence conservation, and upstream regulation by repeated motifs (Yan et al., 1999; Martinez-Calvillo et al., 2001). Transcription of rRNA in Leishmania is driven by RNAP I. The RNA polymerase III promoters are responsible for the transcription of tRNAs and small RNAs (Uliana et al., 1996).

Regulation of gene expression is an array of various mechanisms that cells use in order to increase or decrease the production of specific gene products, such as RNA or proteins. Leishmania is considered to be one of the most ancient eukaryotes. Compared to other eukaryotes, Leishmania is unusual in terms of gene organization, transcription, and mRNA processing. Three types of RNA polymerases play important roles in the transcription of Leishmania genes. rRNA and tRNA genes are transcribed by RNA polymerase I and III, respectively, whereas RNA polymerase II, which is sensitive to $\alpha$ amanitin, is involved in the transcription of protein-coding genes (Werbovetz, 2008). A remarkable difference exists between gene expression mechanisms of Leishmania and those of the mammalian system, particularly in the processing of pre-mature RNA. First, in mammals, transcription of protein coding genes is monocistronic, either carried out by RNA polymerase (RNAP) II or by each gene's own transcriptional control, whereas in Leishmania, though RNAP II is involved, open reading frames arranged in long arrays are transcribed into polycistronic pre-RNA (RNA that contains multiple protein-coding sequences) that produces monocistronic RNA's through post-transcriptional processing (Clayton and Shapira, 2007) (Figure 14). 


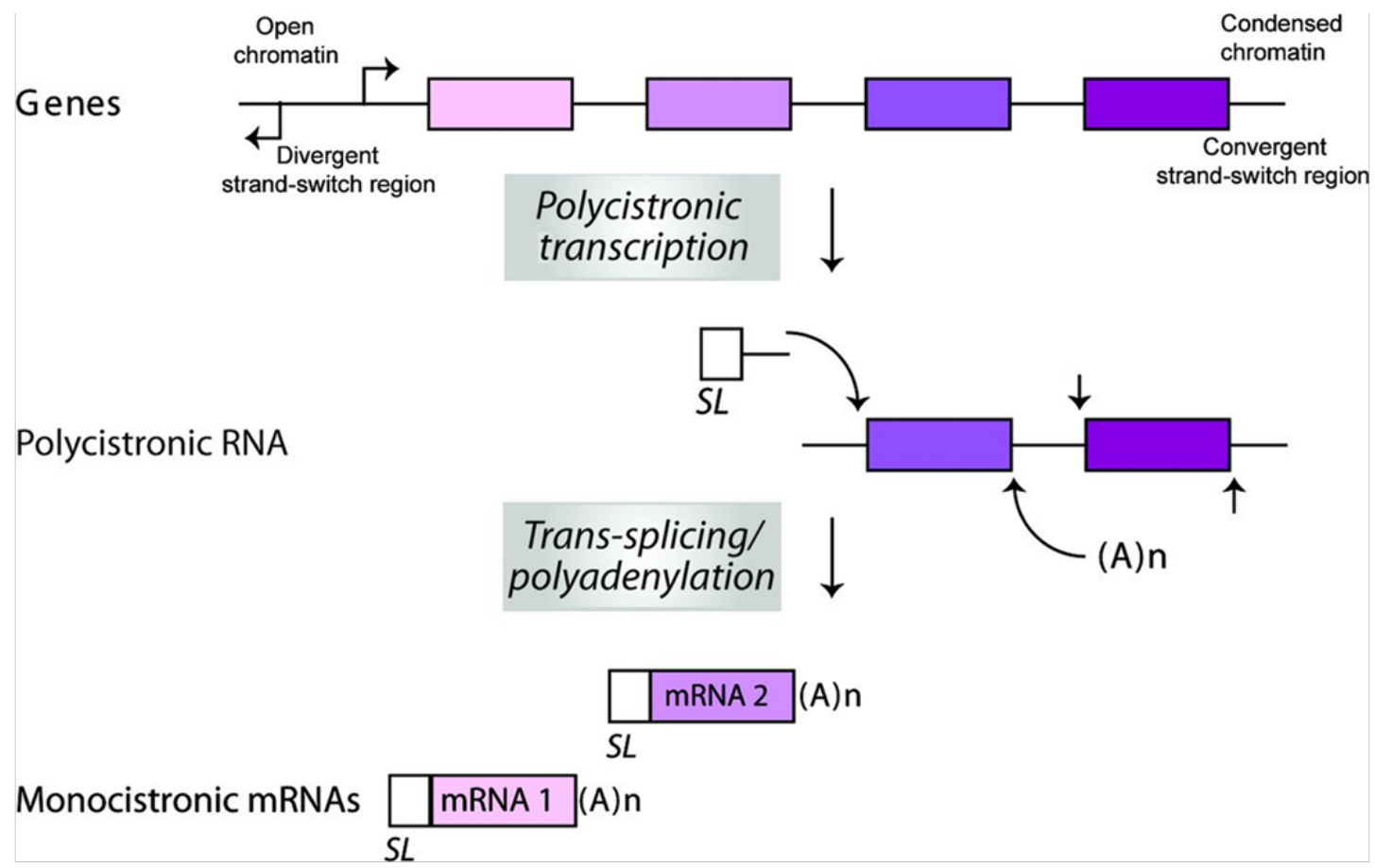

Figure 14. Process of mRNA maturation by trans-splicing and polyadenylation in Leishmania. Transcription initiates at divergent strand-switch regions (open chromatin) and ends at convergent strand-switch regions (condensed chromatin). Clusters of genes coding for proteins of unrelated functions are first transcribed into polycistronic transcripts and then processed by coupled trans-splicing and polyadenylation mechanisms, giving rise to mature monocistronic mRNAs. Boxes represent four different coding sequences and (A)n depicts poly(A) tail (Gaudenzi et al. 2011).

Polycistronic units contain areas known as strand-switch regions (SSRs). These polycistronic units can be convergent (opposite strands containing transcriptional operons converge towards SSRs) or divergent (opposite strands of SSRs diverge from one another), which depend entirely on the transcriptional orientation. In Leishmania, divergent SSRs are associated with transcription initiation, whereas convergent SSRs are the sites for termination of transcription. A second difference is that splicing in mammals is carried out by removal of introns from the same RNA molecule, that is, through the cis-splicing mechanism. In Leishmania, a trans-splicing mechanism and polyadenylation 
are the two coupled cleavage reactions, which are responsible for the processing of polycistronic pre-mRNA. Trans-splicing mechanisms occur when exons are fused from two different RNA molecules and lack the presence of introns (Figure 14) (Haile and Papadopoulou, 2007).

During a trans-splicing reaction, a small capped RNA, known as the splice leader RNA (SL RNA), which is 39-41 nucleotides in length, is added to the 5' end of all known protein-coding RNAs, followed by introns of variable length which can be folded into two stem loops separated by a single strand region (Clayton and Shapira, 2007). Transsplicing also occurs through two-step trans-esterification, forming a Y- shaped structure rather than the lariat formed in eukaryotes (Figure 15). 


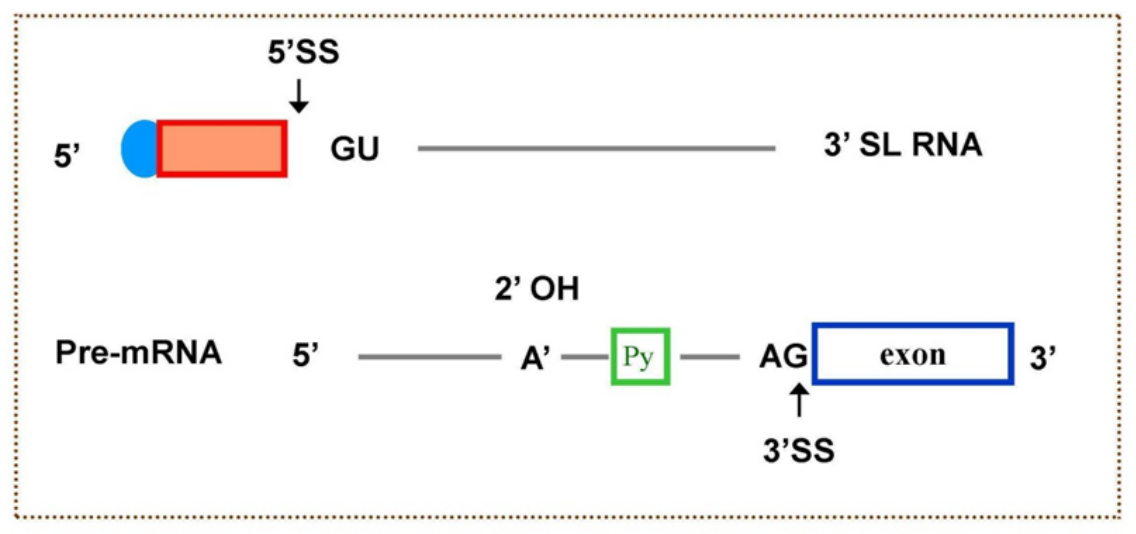

FIRST STEP

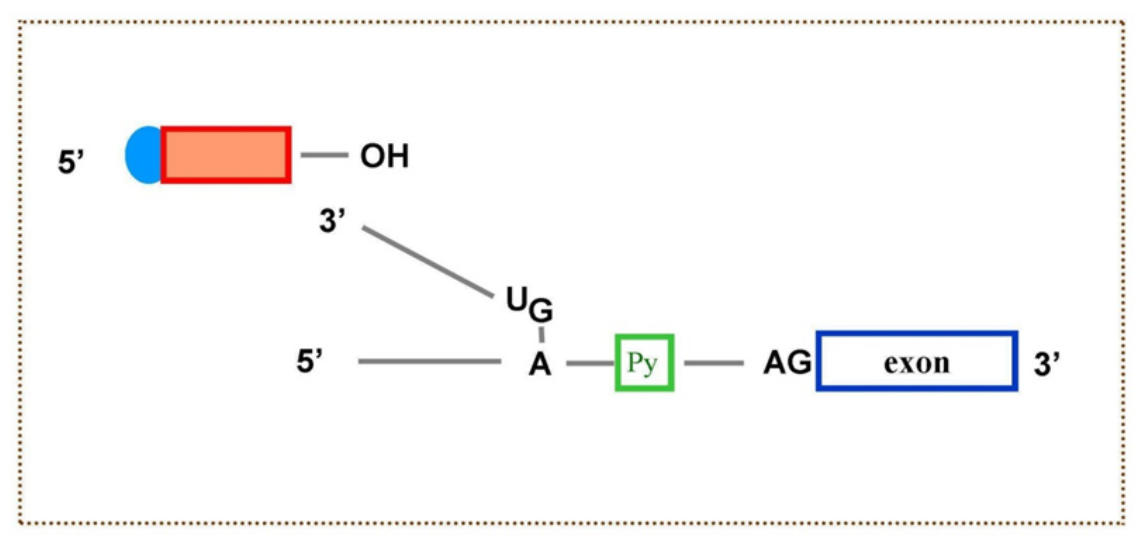

SECOND STEP

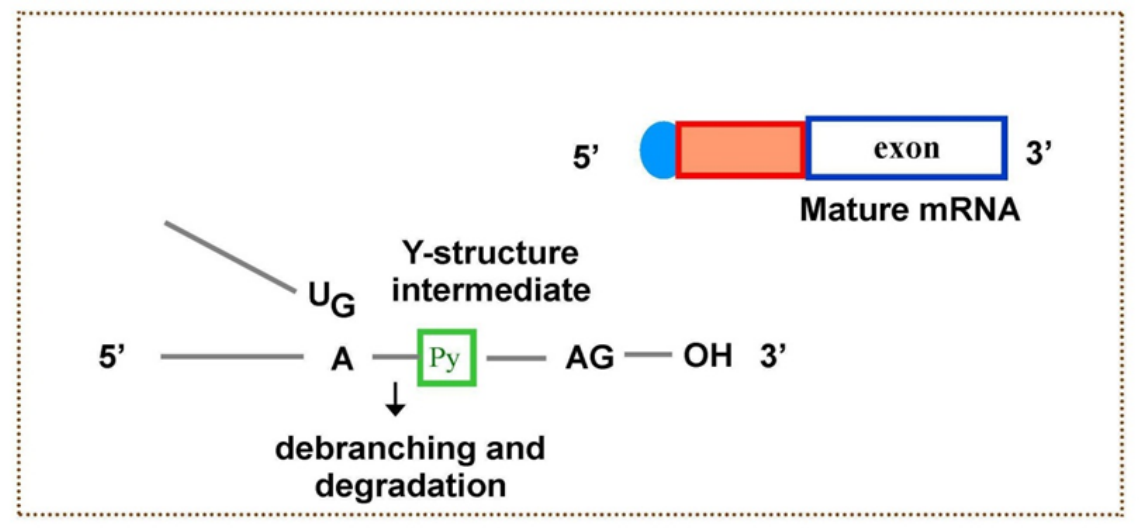

Figure 15. Mechanism of trans-splicing. The 5' splice site GU on the SL RNA and the 3' splice site AG on the pre-mRNA are indicated. BP, branch point; Py, polypyrimidine tract (Liang et al. 2003). 
The presence of a hypermodified cap structure, known as cap 4, is the most distinctive feature of SL RNA because the 4 nt present after the 7- methylguanosine $\left(\mathrm{m}^{7} \mathrm{G}\right)$ are modified. Therefore, the addition of SL performs two functions: (a) SL sequence and polyadenylation together help in the separation of polycistronic transcripts, and (b) SL sequence provides a cap to mRNA (Liang et al., 2003). Trans-splicing signals are usually U-rich polypyrimidine (poly (Y)) tracts, preceded by an AG splice acceptor site 50-100 nt upstream of the translational start codon. In Leishmania, the 3' splice acceptor site is an AG dinucleotide, which is present downstream to the polypyrimidine tract (Haile and Papadopoulou, 2007). The polypyrimidine tracts within intergenic regions play important roles in the coupling of these processes in Leishmania and trypanosome transcripts. Hug et al. (1994) reported that mutation in the poly (Y) tract leads to an aberrant poly (A) site choice. There are several gaps in the understanding of pre-mRNA processing in trypanosomes. The major question is how the selection of exact 3' splice site upstream of the translation initiator start codon takes place. It was suggested that the factors required for pre-mRNA processing in Leishmania are marked by spliceosome machinery after the 3'splice site region; these factors may also direct polyadenylation upstream (Matthews et al., 1994). Studies by Lebowitz et al. (1993) suggested that the poly (A) site shifts 400-500 nt upstream of the new splice site whenever the position of the downstream splice site is altered. Therefore, selection of the poly (A) site is specified by the position of the downstream splice acceptor site in Leishmania. Since trans-splicing and polyadenylation are coupled processes, polyadenylation sites are present 100-400 nt upstream from the trans-splicing signal and are dependent on downstream splicing (Liang et al., 2003). These post-transcriptional 
RNA processing reactions are essential to mRNA maturation but may also help to control gene expression.

In eukaryotes, internal ribosome entry sites (IRES) and upstream ORFs present in 5'UTRs of mRNA are critical to regulation of mRNAs translation in response to environmental stimuli (Meijer and Thomas, 2002). The 5'- untranslated regions (5'UTRs) of mRNA are generally short in Leishmania because of the lack of IRES and upstream ORFs (Meijer and Thomas, 2002), whereas the sequences present within the 3'untranslated regions (3'UTR) regulate differentially expressed genes in Leishmania and are reported to be involved in stage-specific post-transcriptional gene regulation (Argaman et al., 1994). Unusually long 3'UTR sequences and lack of promoter elements for RNA polymerase II provide evidence of the molecular basis for this type of control. In general, gene regulation in trypanosomes occurs via post-transcriptional and/or posttranslational mechanisms, which include mRNA processing, mRNA degradation and translational efficiency, and protein processing, modification and stability (Papadopoulou et al., 2003). Regulatory mechanisms affecting global and specific gene expression in Leishmania are activated due to extreme environmental changes during the conversion from promastigotes to amastigotes. In Leishmania chagasi, studies reported that the regulation of genes encoding major surface protease (MSPL) is mediated by a $202 \mathrm{nt}$ region present within the MSPL 3'UTR, which confers an increase in mRNA stability (Purdy et al., 2005). Kelly et al. (2001) observed that in L. major, stage-specific leishmanolysin genes (GP63) are under the influence of sequences within the 3'UTR. Several studies suggest that translation efficiencies are governed by the sequences present 
in the 3'UTR (Papadopoulou et al., 2003). Certain experimental evidence suggested that the 3'UTR bearing heat shock protein (HSP70) transcripts (HSP70-I) were found to be associated with ribosomes in promastigotes at normal and heat-shock temperatures, whereas HSP70-II transcripts appeared to preferentially translated at heat-shock temperatures but not at $26^{\circ} \mathrm{C}$ (temperature inside sandflies) (Folgueira et al., 2005). In trypansomatids, the first evidence of the presence of sequences within $770 \mathrm{nt}$ of the amastin 3'UTR was reported, implicating the involvement of these sequences for stagespecific regulation. On the basis of the presence of sequences in amastin 3'UTR, Papadopoulou et al. (2003) identified the presence of 450 nt 3'UTR element in the amastin mRNA in L. major and its role in regulating mRNA translation by enabling the 3'UTR element to bind tightly with polysomes in a stage-specific manner. It was shown that regulation through the $450 \mathrm{nt}$ element is triggered in response to heat shock. However, this element is not conserved in all the amastigote specific transcripts. Stagespecific gene expression can be controlled either at the level of mRNA stability or at the translation level by a bipartite mechanism, which involves 3'UTR elements that mediate regulation at the level of mRNA abundance and translation, respectively, in Leishmania (Papadopoulou et al., 2003). It was reported that this 3’UTR element belongs to a new family of extinct short retroposons, small-interspersed degenerate retroposons (SIDER1) (Papadopoulou et al., 2003). The other two amastin transcripts bearing a 3'UTR element of 500-550 nt (Rochette et al., 2005) were parts of another SIDER subfamily, SIDER 2, which is distinct from SIDER1 in terms of promoting mRNA stabilization. The 3'UTR elements classified to date in Leishmania are different from the other known regulatory elements previously reported in eukaryotes, including trypanosomes. It was found that 
regulation of gene expression is complex in Leishmania and is controlled by a diversity of mechanisms, as the transcripts contain more than one regulatory element in the 3'UTR. In trypanosomatids, RNA-binding proteins (RBP) are key factors in regulating gene expression. Over one hundred RBPs have been predicted to exist in trypanosomes, based on the presence of canonical RNA-binding domains: RNA recognition motif (RRM), CCCH zinc fingers, and Pumilio or PUF domains. Most of the characterized RBPs control mRNA abundance by stabilizing the target transcript (De Gaudenzi et al. ,2011) . In trypanosomatids, although indirect results suggest that some proteins cause degradation of target transcripts, this has never been shown directly (Erben et al., 2014).

Phosphorylation, methylation, acetylation or glycosylation are some of the posttranslational events, which are responsible for the functional changes in proteins. Furthermore, ubiquitination, another post-translational event, is responsible for the proteasome-dependent degradation pathway of protein. Several post-translational modifications have been reported in trypanosomatids but post- translational regulation of proteins has not yet been abundant and there are missing links. In Leishmania, environmental changes lead to modulation in protein phosphorylation or protein kinase activities. Studies reported that in virulent $L$. donovani promastigotes, heat stress stimulates the alteration in protein phosphorylation (Salotra et al., 2000) whereas in $L$. pifanoi, protein tyrosine phosphorylation is activated by acidic $\mathrm{pH}$ (Rivero-Lezcano et al., 1997). In L. major, endogenous membrane protein phosphorylation have been observed during the differentiation from promastigotes to metacyclic forms (Hermoso and Jaffe 1993). In L. mexicana, Wiese et al., 2003 showed that mitogen-activated protein kinase 
kinase (MAPKK) homologue which is regulated by phosphorylation is involved in the regulation of flagellar assembly and cell size. There are no reports about such regulations of aquaporins in trypanosomatids. Since, AQP1 is directly related to drug resistance understanding the regulation will help us to combat resistance more efficiently.

\subsection{Rationale of the study}

The long-term goal of this project is to decipher the signaling cascade of AQP1 channel regulation in order to combat antimonial resistance. The current objectives of this project are to identify the immediate players involved in the regulation of AQP1 channel function. Our central hypothesis is that the Leishmania aquaglyceroporin AQP1 is regulated at the post-transcriptional and post-translational levels through interaction with other cellular components. We plan to dissect this interplay step-by-step. We demonstrated that Leishmania MAPK2 positively regulate AQP1 and localized it to the pellicular membrane by phosphorylating a cytosolic loop D residue Threonine 197 (Mandal et al., 2012). The proposed work has provided an enhanced understanding of the fundamental mechanisms by which an aquaglyceroporin helps a parasite to sense its environment, including antimonial exposure. These are unchartered areas of parasite biology and physiological functions of aquaglyceroporins. The following specific aims have examined how AQP1 is regulated at the a) post-translational and b) posttranscriptional levels: 


\subsubsection{Deciphering the mechanism (s) of regulation of AQP1 at the post- translational level:}

Detailed analysis of AQP1 protein sequence by unique software and our data revealed evidence of ubiquitination sites. Inhibitory and site-directed mutagenesis studies along with tandem affinity tag methodology were used to dissect these mechanisms. The split-ubiquitin membrane yeast two hybrid systems was an additional approach to unravel the AQP1 interactome.

\subsubsection{Elucidating the mechanism (s) of post-transcriptional regulation of AQP1:}

Our data indicate that the level of AQP1 expression is regulated at the posttranscriptional level by the 3'UTR and is species-specific. Mechanisms were studied through luciferase reporter assay.

The Mukhopadhyay's group was the first to identify and characterize the Leishmania aquaglyceroporin AQP1 and its involvement in parasite physiology. Identification of specific novel features of this AQP has been a major contribution to the Leishmania and aquaporin fields as represented by the fact that our work was featured by "International Innovation" in their latest special edition on "Women in Science"- A Passion for Progress. Collectively, these studies explore a novel aspect of parasite biology and a unique aquaglyceroporin. The novel aspect of unique aquaglyceroporin will have a positive impact on our understanding of Leishmania physiology and how it helps a parasite to survive in a stressful environment and facilitate antimonial uptake. Our evidence suggests that AQP1 is more intricately involved with various cellular machinery and signal transduction than previously thought. Since AQP1 is essential for 
parasite survival, our eventual long-term goal is to target AQP1 and its regulatory elements to resolve antimonial resistance and to identify new targeted interventions. 


\subsection{References}

Alvar, J., Aparicio, P., Aseffa, A., Den Boer, M., Cañavate, C., Dedet, J. P., et al. (2008). The relationship between leishmaniasis and AIDS: the Second 10 Years. Clinical Microbiology Reviews, 21(2), 334-359.

Amato, V. S., Tuon, F. F., Bacha, H. l. A., Neto, V. A., \& Nicodemo, A. C. (2008). Mucosal leishmaniasis: Current scenario and prospects for treatment. Acta Tropica, 105(1), 1-9.

Argaman, M., Aly, R., \& Shapira, M. (1994). Expression of heat shock protein 83 in Leishmania is regulated post-transcriptionally. Molecular and Biochemical Parasitology, 64(1), 95-110.

Ashutosh, Sundar, S., \& Goyal, N. (2007). Molecular mechanisms of antimony resistance in Leishmania. Journal of Medical Microbiology, 56(2), 143-153.

Beitz, E. (2005). Aquaporins from pathogenic protozoan parasites: structure, function and potential for chemotherapy. Biology of the Cell, 97(6), 373-383.

Beitz, E. (2006). Aquaporin water and solute channels from malaria parasites and other pathogenic protozoa. ChemMedChem, 1(6), 587-592.

Berman, J. D., Waddell, D., \& Hanson, B. D. (1985). Biochemical mechanisms of the antileishmanial activity of sodium stibogluconate. Antimicrobial Agents and Chemotherapy, 27(6), 916-920.

Bittencourt, A. a., Silva, N., Straatmann, A. a., Nunes, V. L. C., Follador, I., \& Badaro, R. (2002). Post-kala-azar dermal leishmaniasis associated with AIDS. Brazilian Journal of Infectious Diseases, 6, 313-316.

Borst, P., \& Ouellette, M. (1995). New mechanisms of drug resistance in parasitic protozoa. Annual Reviews in Microbiology, 49(1), 427-460.

Bringaud, F. D. R, Ghedin, E., Blandin, G. 1., Bartholomeu, D. C., Caler, E., Levin, M. J., et al. (2006). Evolution of non-LTR retrotransposons in the trypanosomatid genomes: Leishmania major has lost the active elements. Molecular and Biochemical Parasitology, 145(2), 158-170.

Callahan, H.L. \& Beverley , S.M. (1991). Heavy metal resistance: a new role for Pglycoproteins in Leishmania. Journal of Biological Chemistry, 266(28), 1842730 . 
Chakraborty, A. K., \& Majumder, H. K. (1988). Mode of action of pentavalent antimonials: Specific inhibition of type I DNA topoisomerase of Leishmania donovani. Biochemical and Biophysical Research Communications, 152(2), 605611.

Choi, C. M., \& Lerner, E. A. (2001). Leishmaniasis as an emerging infection. Journal of Investigative Dermatology Symposium Proceedings, 6(3), 175-182.

Clayton, C., \& Shapira, M. (2007). Post-transcriptional regulation of gene expression in trypanosomes and Leishmania. Molecular and Biochemical Parasitology, 156(2), 93-101.

Croan, D. G., Morrison, D. A., \& Ellis, J. T. (1997). Evolution of the genus Leishmania revealed by comparison of DNA and RNA polymerase gene sequences. Molecular and Biochemical Parasitology, 89(2), 149-159.

De Gaudenzi, J. G., Noe, G., Campo, V. A., Frasch, A. C., \& Cassola, A. (2011). Gene expression regulation in trypanosomatids. Essays In Biochemistry, 51, 31-46.

Decuypere, S., Rijal, S., Yardley, V., De Doncker, S., Laurent, T., Khanal, B., et al. (2005). Gene expression analysis of the mechanism of natural Sb(V) resistance in Leishmania donovani isolates from Nepal. Antimicrobial Agents and Chemotherapy, 49(11), 4616-4621.

Denton, H., McGregor, Joanne C., \& Coombs, Graham H. (2004). Reduction of antileishmanial pentavalent antimonial drugs by a parasite-specific thiol-dependent reductase, TDR1. Biochemical Journal, 381(Pt 2), 405-412.

Dey, S., Papadopoulou, B., Haimeur, A., Roy, G., Grondin, K., Dou, D., Rosen, B.P., \& Ouellette, M. (1994). High level arsenite resistance in Leishmania tarentolae is mediated by an active extrusion system. Molecular and Biochemical Parasitology, 67(1), 49-57.

Dooijes, D., Chaves, I. S., Kieft, R., Dirks-Mulder, A., Martin, W., \& Borst, P. (2000). Base $\mathrm{J}$ originally found in Kinetoplastida is also a minor constituent of nuclear DNA of Euglena gracilis. Nucleic Acids Research, 28(16), 3017-3021.

El Fadili, K., Messier, N., Leprohon, P., Roy, G. t., Guimond, C., Trudel, N., et al. (2005). Role of the ABC transporter MRPA (PGPA) in antimony resistance in Leishmania infantum axenic and intracellular amastigotes. Antimicrobial Agents and Chemotherapy, 49(5), 1988-1993.

Engel, A., \& Stahlberg, H. (2002). Aquaglyceroporins: Channel proteins with a conserved core, multiple functions, and variable surfaces. International Review of Cytology, 215, 75-104. 
Erben, E. D., Fadda, A., Lueong, S., Hoheisel, J. R. D., \& Clayton, C. (2014). A genomewide tethering screen reveals novel potential post-transcriptional regulators in Trypanosoma brucei. PLoS Pathogens, 10(6), 1004178.

Jeddi, F., Piarroux, R. Mary,C. (2011). Antimony resistance in Leishmania, focusing on experimental research. Journal of Tropical Medicine, 10(1155), 695382.

Figarella, K., Uzcategui, N. L., Zhou, Y., LeFurgey, A., Ouellette, M., Bhattacharjee, H., et al. (2007). Biochemical characterization of Leishmania major aquaglyceroporin LmAQP1: possible role in volume regulation and osmotaxis. Molecular Microbiology, 65(4), 1006-1017.

Folgueira, C., Quijada, L., Soto, M., Abanades, D. R., Alonso, C., \& Requena, J. M. (2005). The translational efficiencies of the two Leishmania infantum HSP70 mRNAs, differing in their 3'-untranslated regions, are affected by shifts in the temperature of growth through different mechanisms. Journal of Biological Chemistry, 280(42), 35172-35183.

Fu, G., \& Barker, D. C. (1998). Characterisation of Leishmania telomeres reveals unusual telomeric repeats and conserved telomere-associated sequence. Nucleic Acids Research, 26(9), 2161-2167.

Gilinger, G., \& Bellofatto, V. (2001). Trypanosome spliced leader RNA genes contain the first identified RNA polymerase II gene promoter in these organisms. Nucleic Acids Research, 29(7), 1556-1564.

Goodwin, L. G. (1995). Pentostam ${ }^{\circledR}$ (sodium stibogluconate); a 50-year personal reminiscence. Transactions of The Royal Society of Tropical Medicine and Hygiene, 89(3), 339-341.

Gourbal, B., Sonuc, N., Bhattacharjee, H., Legare, D., Sundar, S., Ouellette, M., et al. (2004). Drug uptake and modulation of drug resistance in Leishmania by an aquaglyceroporin. Journal of Biological Chemistry, 279(30), 31010-31017.

Grondin, K., Papadopoulou, B., \& Ouellette, M. (1993). Homologous recombination between direct repeat sequences yields P-glycoprotein containing amplicons in arsenite resistant Leishmania. Nucleic Acids Research, 21(8), 1895-1901.

Haile, S., \& Papadopoulou, B. (2007). Developmental regulation of gene expression in trypanosomatid parasitic protozoa. Current Opinion in Microbiology, 10(6), 569577.

Haimeur, A., Brochu, C., Genest, P. A., Papadopoulou, B., \& Ouellette, M. (2000). Amplification of the ABC transporter gene PGPA and increased trypanothione 
levels in potassium antimonyl tartrate (SbIII) resistant Leishmania tarentolae. Molecular and Biochemical Parasitology, 108(1), 131-135.

Haimeur, A., Guimond, C., Pilote, S., Mukhopadhyay, R., Rosen, B. P., Poulin, R., et al. (1999). Elevated levels of polyamines and trypanothione resulting from overexpression of the ornithine decarboxylase gene in arsenite-resistant Leishmania. Molecular Microbiology, 34(4), 726-735.

Haldar, J. P., Ghose, S., Saha, K. C., \& Ghose, A. C. (1983). Cell-mediated immune response in Indian kala-azar and post-kala-azar dermal leishmaniasis. Infection and Immunity, 42(2), 702-707.

Hara-Chikuma, M., \& Verkman, A. S. (2006). Physiological roles of glyceroltransporting aquaporins: the aquaglyceroporins. Cellular and Molecular Life Sciences CMLS, 63(12), 1386-1392.

Hermoso, T., \& Jaffe, C. L. (1993). Phosphorylation of proteins in virulent promastigotes from Leishmania major. Biological research, 26(1-2), 267-271.

Herwaldt, B. L. (1999). Leishmaniasis. The Lancet, 354(9185), 1191-1199.

Hub, J. S., \& de Groot, B. L. (2008). Mechanism of selectivity in aquaporins and aquaglyceroporins. Proceedings of the National Academy of Sciences, 105(4), 1198-1203.

Hug, M., Hotz, H. R., Hartmann, C., \& Clayton, C. (1994). Hierarchies of RNAprocessing signals in a trypanosome surface antigen mRNA precursor. Molecular and Cellular Biology, 14(11), 7428-7435.

Ivens, A. C., Peacock, C. S., Worthey, E. A., Murphy, L., Aggarwal, G., Berriman, M., et al. (2005). The genome of the kinetoplastid parasite, Leishmania major. Science (New York, N.Y.), 309(5733), 436-442.

Jensen, M. O., Park, S., Tajkhorshid, E., \& Schulten, K. (2002). Energetics of glycerol conduction through aquaglyceroporin GlpF. Proceedings of the National Academy of Sciences, 99(10), 6731-6736.

Kamhawi, S. (2006). Phlebotomine sand flies and Leishmania parasites: friends or foes? Trends in Parasitology, 22(9), 439-445.

Kelly, B. L., Nelson, T. N., \& McMaster, W. R. (2001). Stage-specific expression in Leishmania conferred by 3 ' untranslated regions of L. major leishmanolysin genes (GP63). Molecular and Biochemical Parasitology, 116(1), 101-104. 
LeBowitz, J. H., Smith, H. Q., Rusche, L., \& Beverley, S. M. (1993). Coupling of poly(A) site selection and trans-splicing in Leishmania. Genes \& Development, 7(6), 996-1007.

Lègarè, D., Richard, D., Mukhopadhyay, R., Stierhof, Y. D., Rosen, B. P., Haimeur, A., et al. (2001). The Leishmania ATP-binding cassette protein PGPA is an intracellular metal-thiol transporter ATPase. Journal of Biological Chemistry, 276(28), 26301-26307.

Lesho, E.P., Wortmann, G., Neafie, R.C., \& Aronson, N.E. (2004). Cutaneous leishmaniasis: Battling the baghdad boil. Continuing Medical Education and Continuing Education.

Leprohon, P., Lègarè, D., \& Ouellette, M. (2009). Intracellular localization of the ABCC proteins of Leishmania and their role in resistance to antimonials. Antimicrobial Agents and Chemotherapy, 53(6), 2646-2649.

Liang, X.h., Haritan, A., Uliel, S., \& Michaeli, S. (2003). Trans and Cis Splicing in Trypanosomatids: Mechanism, Factors, and Regulation. Eukaryotic Cell, 2(5), 830-840.

Lodish H, Berk A, Zipursky SL, et al. (2000). Molecular Cell Biology. 4th edition. New York: W.H.Freeman.

Lugo de Yarbuh, A., Anez, N., Petit de Pena, Y., Burquera, J.L., \& Burquera, M. (1994) Antimony determination in tissues and serum of hamsters infected with Leishmania garnhami and treated with meglumine antimoniate. Annals of Tropical Medicine and Parasitology, 88(1), 37-41

Lukes, J., Mauricio, I. L., Schönian, G., Dujardin, J.C., Soteriadou, K., Dedet, J.P., et al. (2007). Evolutionary and geographical history of the Leishmania donovani complex with a revision of current taxonomy. Proceedings of the National Academy of Sciences of the United States of America, 104(22), 9375-9380.

Mandal, G., Mandal, S., Sharma, M., Charret, K. S., Papadopoulou, B., Bhattacharjee, H., et al. (2015). Species-specific antimonial sensitivity in Leishmania is driven by post-transcriptional regulation of AQP1. PLoS Neglected Tropical Diseases, 9(2), 0003500 .

Mandal, G., Govindarajan, V., Sharma, M., Bhattacharjee, H., and Mukhopadhyay, R. Drug resistance in Leishmania (2014). In. Antimicrobial drug resistance: Principles and Practice for the Clinic and Bench. Eds, M. Ouellette and S. Lerner, Springer. 
Mandal, G., Orta, J., Sharma, M., \& Mukhopadhyay, R. (2014). Trypanosomatid aquaporins: Roles in physiology and drug response. Diseases, 2(1), 3.

Mandal, G., Sharma, M., Kruse, M., Sander-Juelch, C., Munro, L. A., Wang, Y., et al. (2012). Modulation of Leishmania major aquaglyceroporin activity by a mitogenactivated protein kinase. Molecular Microbiology, 85(6), 1204-1218.

Mandal, S., Maharjan, M., Singh, S., Chatterjee, M., \& Madhubala, R. (2010). Assessing aquaglyceroporin gene status and expression profile in antimony-susceptible and resistant clinical isolates of Leishmania donovani from India. Journal of Antimicrobial Chemotherapy, 65(3), 496-507.

Manzano, J. I., García-Hernández, R., Castanys, S., \& Gamarro, F. (2013). A new ABC half-transporter in Leishmania major is involved in resistance to antimony. Antimicrobial Agents and Chemotherapy, 57(8), 3719-3730.

Martinez-Calvillo, S., Sunkin, S. M., Yan, S.f., Fox, M., Stuart, K., \& Myler, P. J. (2001). Genomic organization and functional characterization of the Leishmania major friedlin ribosomal RNA gene locus. Molecular and Biochemical Parasitology, 116(2), 147-157.

Matthews, K. R., Tschudi, C., \& Ullu, E. (1994). A common pyrimidine-rich motif governs trans-splicing and polyadenylation of tubulin polycistronic pre-mRNA in trypanosomes. Genes \& Development, 8(4), 491-501.

Mehta, A., \& Shaha, C. (2006). Mechanism of metalloid-induced death in Leishmania spp.: Role of iron, reactive oxygen species, $\mathrm{Ca}^{2+}$, and glutathione. Free Radical Biology and Medicine, 40(10), 1857-1868.

Meijer, H. A., \& Thomas, A. A. M. (2002). Control of eukaryotic protein synthesis by upstream open reading frames in the 5'-untranslated region of an mRNA. Biochemical Journal, 367(1), 1-11.

Momen, H., \& Cupolillo, E. (2000). Speculations on the origin and evolution of the genus Leishmania. Memòrias do Instituto Oswaldo Cruz, 95, 583-588.

Monge-Maillo, B. a., Norman, F. F., Cruz, I., Alvar, J., \& Lòpez-Vèlez, R. (2014). Visceral leishmaniasis and HIV coinfection in the mediterranean region. PLoS Negl Trop Dis, 8(8), 3021.

Mookerjee Basu, J., Mookerjee, A., Sen, P., Bhaumik, S., Sen, P., Banerjee, S., et al. (2006). Sodium antimony gluconate induces generation of reactive oxygen species and nitric oxide via phosphoinositide 3-kinase and mitogen-activated protein kinase activation in Leishmania donovani infected macrophages. Antimicrobial Agents and Chemotherapy, 50(5), 1788-1797. 
Mukhopadhyay, R., Dey, S., Xu, N., Gage, D., Lightbody, J., Ouellette, M., et al. (1996). Trypanothione overproduction and resistance to antimonials and arsenicals in Leishmania. Proceedings of the National Academy of Sciences of the United States of America, 93(19), 10383-10387.

Mukhopadhyay, R., Mandal, G., Atluri, V. S. R., Figarella, K., Uzcategui, N. L., Zhou, Y., et al. (2011). The role of alanine 163 in solute permeability of Leishmania major aquaglyceroporin LmAQP1. Molecular and Biochemical Parasitology, 175(1), 83-90.

Noyes, H. (1998). Implications of a neotropical origin of the genus Leishmania. Memòrias do Instituto Oswaldo Cruz, 93, 657-662.

Ouellette, M., Haimeur, A., Grondin, K., Lègarè, D., \& Papadopoulou, B. (1998). Amplification of $\mathrm{ABC}$ transporter gene pgpA and of other heavy metal resistance genes in Leishmania tarentolae and their study by gene transfection and gene disruption. Methods in Enzymology, 292, 182-193.

Ouellette, M., Hettema, E., Wüst, D., Fase-Fowler, F., \& Borst, P. (1991). Direct and inverted DNA repeats associated with P-glycoprotein gene amplification in drug resistant Leishmania. The EMBO Journal, 10(4), 1009-1016.

Papadopoulou, B., Roy, G., Dey, S., Rosen, B. P., \& Ouellette, M. (1994). Contribution of the Leishmania P-glycoprotein-related gene pgpA to oxyanion resistance. Journal of Biological Chemistry, 269(16), 11980-11986.

Papadopoulou, B., Huang, X.F., Boucher, N., \& McNicoll, F. (2003). Stage-specific regulation of gene expression in Leishmania. ASM news, 69.

Purdy, J. E., Donelson, J. E., \& Wilson, M. E. (2005). Regulation of genes encoding the major surface protease of Leishmania chagasi via mRNA stability. Molecular and Biochemical Parasitology, 142(1), 88-97.

Ramalho-Ortigão, J. M., Kamhawi, S., Joshi, M. B., Reynoso, D., Lawyer, P. G., Dwyer, D. M., et al. (2005). Characterization of a blood activated chitinolytic system in the midgut of the sand fly vectors Lutzomyia longipalpis and Phlebotomus papatasi. Insect Molecular Biology, 14(6), 703-712.

Reithinger, R., Dujardin, J.C., Louzir, H., Pirmez, C., Alexander, B., \& Brooker, S. (2007). Cutaneous leishmaniasis. The Lancet Infectious Diseases, 7(9), 581-596.

Rivero-Lezcano, O. M., Chicharro, C., \& Rivas, L. (1997). Acidic pH stress induces protein tyrosine phosphorylation in Leishmania pifanoi. Molecular and Biochemical Parasitology, 84(1), 123-129. 
Rochette, A., McNicoll, F. O., Girard, J., Breton, M., Leblanc, A. R., Bergeron, M. G., et al. (2005). Characterization and developmental gene regulation of a large gene family encoding amastin surface proteins in Leishmania spp. Molecular and Biochemical Parasitology, 140(2), 205-220.

Roger, A. J., \& Hug, L. A. (2006). The origin and diversification of eukaryotes: problems with molecular phylogenetics and molecular clock estimation. Philosophical Transactions of the Royal Society B: Biological Sciences, 361(1470), 1039-1054.

Salotra, P., Ralhan, R., \& Sreenivas, G. (2000). Heat-stress induced modulation of protein phosphorylation in virulent promastigotes of Leishmania donovani. The International Journal of Biochemistry \& Cell Biology, 32(3), 309-316.

Savoia, D. (2015). Recent updates and perspectives on leishmaniasis. Journal of Infection in Developing countries, 9(6), 588- 596.

Sereno, D., Cavaleyra, M., Zemzoumi, K., Maquaire, S., Ouaissi, A., \& Lemesre, J. L. (1998). Axenically grown amastigotes of Leishmania infantum used as an in vitro model to investigate the pentavalent antimony mode of action. Antimicrobial Agents and Chemotherapy, 42(12), 3097-3102.

Sereno, D., Holzmuller, P., Mangot, I., Cuny, G. R., Ouaissi, A., \& Lemesre, J. L. (2001). Antimonial-mediated DNA fragmentation in Leishmania infantum amastigotes. Antimicrobial Agents and Chemotherapy, 45(7), 2064-2069.

Sereno, D., \& Lemesre, J. L. (1997). Axenically cultured amastigote forms as an in vitro model for investigation of antileishmanial agents. Antimicrobial Agents and Chemotherapy, 41(5), 972-976.

Shaked-Mishan, P., Ulrich, N., Ephros, M., \& Zilberstein, D. (2001). Novel intracellular $\mathrm{Sb}(\mathrm{V})$ reducing activity correlates with antimony susceptibility in Leishmania donovani. Journal of Biological Chemistry, 276(6), 3971-3976.

Song, J., Mak, E., Wu, B., \& Beitz, E. (2014). Parasite aquaporins: Current developments in drug facilitation and resistance. Biochimica et Biophysica Acta, 1840(5), 15661573.

Sundar, S., Jha, T. K., Thakur, C. P., Sinha, P. K., \& Bhattacharya, S. K. (2007). Injectable paromomycin for visceral leishmaniasis in India. New England Journal of Medicine, 356(25), 2571-2581.

Sundar, S., \& Rai, M. (2002). Laboratory diagnosis of visceral leishmaniasis. Clinical and Diagnostic Laboratory Immunology, 9(5), 951-958. 
Sundar, S., Sinha, P. K., Rai, M., Verma, D. K., Nawin, K., Alam, S., et al. (2011). Comparison of short-course multidrug treatment with standard therapy for visceral leishmaniasis in India: an open-label, non-inferiority, randomised controlled trial. The Lancet, 377(9764), 477-486.

Tosato, V., Ciarloni, L., Ivens, A., Rajandream, M. A. l., Barrell, B., \& Bruschi, C. (2001). Secondary DNA structure analysis of the coding strand switch regions of five Leishmania major Friedlin chromosomes. Current Genetics, 40(3), 186-194.

Uliana, S. R. B., Fischer, W., Stempliuk, V. A., \& Floeter-Winter, L. M. (1996). Structural and functional characterization of the Leishmania amazonensis ribosomal RNA promoter. Molecular and Biochemical Parasitology, 76(12), 245255.

Uliana SRB, Ruiz JC, Cruz AK. Leishmania Genomics: Where Do We Stand? (2006) Bioinformatics in Tropical Disease Research: A Practical and Case-Study Approach. Bethesda (MD): National Center for Biotechnology Information (US); 2008.

Urbina, J. A. (1997). Lipid biosynthesis pathways as chemotherapeutic targets in kinetoplastid parasites. Parasitology, 114(07), 91-99.

Uzcategui, N. L., Zhou, Y., Figarella, K., Ye, J., Mukhopadhyay, R., \& Bhattacharjee, H. (2008). Alteration in glycerol and metalloid permeability by a single mutation in the extracellular C-loop of Leishmania major aquaglyceroporin LmAQP1. Molecular Microbiology, 70(6), 1477-1486.

Von Stebut, E. (2014). Leishmaniasis. Journal der Deutschen Dermatologischen Gesellschaft, 13(3), 191-201.

Werbovetz, K. (2008). Leishmania: After the Genome. Edited by Peter J. Myler and Nicolas Fasel. ChemMedChem, 3(8), 1278-1279.

Wickstead, B., Ersfeld, K., \& Gull, K. (2003). Repetitive elements in genomes of parasitic protozoa. Microbiology and Molecular Biology Reviews, 67(3), 360-375.

Wiese, M., Kuhn, D., \& Grènfelder, C. G. (2003). Protein kinase involved in flagellarlength control. Eukaryotic Cell, 2(4), 769-777.

Wincker, P., Ravel, C., Blaineau, C., Pages, M., Jauffret, Y., Dedet, J. P., et al. (1996). The Leishmania genome comprises 36 chromosomes conserved across widely divergent human pathogenic species. Nucleic Acids Research, 24(9), 1688-1694. 
Yan, S., Lodes, M. J., Fox, M., Myler, P. J., \& Stuart, K. (1999). Characterization of the Leishmania donovani ribosomal RNA promoter. Molecular and Biochemical Parasitology, 103(2), 197-210.

Yurchenko, V. Y., Lukes, J., Jirku, M., Zeledòn, R., \& Maslov, D. A. (2006). Leptomonas costaricensis sp. n. (Kinetoplastea: Trypanosomatidae), a member of the novel phylogenetic group of insect trypanosomatids closely related to the genus Leishmania. Parasitology, 133(05), 537-546.

Zhou, Y., Messier, N., Ouellette, M., Rosen, B. P., \& Mukhopadhyay, R. (2004). Leishmania major LmACR2 is a pentavalent antimony reductase that confers sensitivity to the drug Pentostam. Journal of Biological Chemistry, 279(36), 37445-37451. 


\section{CHAPTER 2}

\section{FUNCTIONAL ROLE OF LYSINE 12 IN LEISHMANIA MAJOR AQP1}

This study has been published:

Sharma, M., Mandal, G., Mandal, S., Bhattacharjee, H., and Mukhopadhyay, R. Functional role of lysine 12 in Leishmania major AQP1 (2015). Molecular and Biochemical Parasitology. Vol 201, 139-145 


\subsection{Introduction}

Leishmaniasis is a vector-borne parasitic disease caused by protozoa of the genus Leishmania. The disease is spread to humans and other mammals by the bite of the female phlebotomine sand fly. Leishmaniasis includes two major diseases, cutaneous leishmaniasis (CL) and visceral leishmaniasis (VL) or kala-azar, caused by more than 20 different Leishmania species. The disease is endemic in parts of 88 countries in five continents - the majority of the affected countries are in the tropics and subtropics. Approximately 2 million new cases occur annually, of which 1.5 million are categorized as CL and 500,000 as VL (Frezard et al., 2009). First-line treatment against this disease consists of antimony V (SbV)-containing drugs, Pentostam or Glucantime. Drug resistance, however, is a major problem in endemic areas. More than $60 \%$ of patients in eastern India are infected with drug-resistant Leishmania parasites (Sundar et al., 2000). The Mukhopadhyay group identified the first Leishmania aquaglyceroporin (AQP1) and demonstrated that modulation of AQP1 determines the sensitivity of the parasite to antimonial drugs (Gourbal et al., 2004; Marquis et al., 2005), which led us to propose that a portion of the pentavalent antimonial drug (SbV) is reduced to trivalent antimony (SbIII) in the macrophage and transported to amastigotes by AQP1. Drug-resistant field isolates from India (Mandal et al., 2010) and Nepal (Decuypere et al., 2005) showed downregulation of AQP1. Because AQP1 is now being used as one of the major drug resistant markers in the endemic regions (Mishra et al., 2012; Kazemi-Rad et al., 2013; Rai et al., 2013), identifying the regulatory mechanisms of AQP1 has the potential to help combat antimonial resistance. AQP1, however, is an adventitious drug transporter. The physiologic functions of this unique AQP are osmoregulation and osmotaxis, which have 
crucial roles in parasite transmission (Figarella et al., 2007). Our recent experiments in the sand fly Lutzomyia longipalpis revealed that $L$. donovani overexpressing AQP1 is more persistent and hyperactive in later stages (e.g., days 11-13) of fly infection compared to wild-type (unpublished data). AQP1 is indispensable for the parasite as it is responsible for guided osmotaxis for coping with the challenges of osmotic stresses. Therefore, manipulation of AQP1 expression levels may have bipartite effect: (i) induction of AQP1 expression may resensitize drug resistant parasites and curtail the antimony toxicity by reducing the current dosage regime, and (ii) inhibition of AQP1 expression may be deleterious to the parasite's survival and transmission.

The Mukhopadhyay group dissected the substrate specificity of the channel and demonstrated that glutamate 152 in the flexible loop C was responsible for selecting between antimony and glycerol (Uzcategui et al., 2008). They defined the pore mouth of AQP1 and showed that alanine 163 and threonine 164 in the extracellular flexible loop C are located very close to the pore mouth (Mukhopadhyay et al., 2011). AQP1 is the first AQP to be exclusively localized in the flagellum of any organism (Figarella et al., 2007). The trypanosomatid flagellum is necessary for parasite migration and invasion of host tissues. Furthermore, the flagellum is proposed to regulate processes such as secretion and endocytosis in the flagellar pocket and may be enriched in proteins involved in environmental or nutritional sensing (Gull, 1999; Bastin et al., 2000; Hill 2003; Vaughan and Gull, 2003). Leishmania promastigotes experience acute changes in osmolarity while thriving inside the sand fly gut or as metacyclics swimming towards the fly's proboscis. Additionally, the parasite faces temperature stress during transmission from 
the sand fly to the vertebrate host and $\mathrm{pH}$ stress while living inside the macrophages in the mammalian host. Antimony exposure is yet another stress when the host is treated with Pentostam or Glucantime. How the parasite senses these crucial environmental changes and transduces these sensations at the molecular level remain gaps in our understanding of parasite biology. We recently showed that species-specific antimonial sensitivity of Leishmania is driven by post-transcriptional regulation which is exclusively mediated by its 3'UTR (Mandal et al., 2015).

Additionally, we previously demonstrated that the induction of Mitogen Activated Protein Kinase 2 (MPK2) from Leishmania increased drug sensitivity by stabilizing AQP1 by phosphorylation, whereas a MAPK2 null mutant was resistant to antimonite. This T197-specific phosphorylation increased the half-life of the channel and also localized AQP1 to the pellicular membrane, giving the parasite a formidable advantage to combat osmotic challenges during and after transmission (Mandal et al., 2012). We also showed that when a variant MPK2 (МРК2к42A), unable to make the phosphoryl transfer, was overexpressed with AQP1, AQP1 was highly destabilized. We speculated that this may be due to a dominant negative effect (Mandal et al., 2012). In this study, we show that this MPK2 к42A mediated accelerated destabilization of AQP1 is due to the presence of a critical lysine 12 (K12) at the disordered cytosolic N-terminus of the protein. When K12A/R-AQP1 was overexpressed with MPK2 ${ }_{\mathrm{K} 42 \mathrm{~A}}$, the Sb(III) facilitator was highly stabilized. The promastigotes co-expressing these altered proteins were hypersensitive to $\mathrm{Sb}(\mathrm{III})$ and accumulated more of the metalloid when compared to AQP1 alone. AQP1 remained confined to the flagellar membrane. 


\subsection{Materials and methods}

\subsubsection{Strains and media}

Leishmania donovani strain LdBob was a kind gift from Dr. Stephen M. Beverley, Washington University School of Medicine. Leishmania promastigotes were grown and maintained in M199 medium as described previously (Mandal et al., 2012). Briefly, the promastigotes were grown at $25^{\circ} \mathrm{C}$ in M199 medium supplemented with $10 \%$ heat inactivated fetal bovine serum (Hyclone), 25mM HEPES (Sigma), 12mM $\mathrm{NaHCO}_{3}$ (Sigma), 2mM glutamine (Life technologies), 1X RPMI 1640 vitamin mix (Sigma), $10 \mu \mathrm{M}$ folic acid (Sigma), $100 \mu \mathrm{M}$ adenosine (Sigma), $7.6 \mathrm{mM}$ hemin (Sigma), 50U/ml penicillin and streptomycin (Life technologies). The $\mathrm{pH}$ was adjusted to 6.8 at $25^{\circ} \mathrm{C}$ with $1 \mathrm{M} \mathrm{NaOH}$. Promastigotes were maintained in $5 \mathrm{ml}$ of M199 medium in a T25 flask (Corning) by serially passaging at a 1:50 dilution in the same medium after every 3 days (Goyard et al., 2003). Table 1 details the constructs, strains and transfectants used in this study. 
Table 1. Plasmids and strains

\begin{tabular}{|c|c|c|}
\hline Plasmids/Strains & Genotype/Description & Reference \\
\hline \multicolumn{3}{|l|}{ Plasmids } \\
\hline pSP72 $\alpha$ hygro $\alpha$ & Leishmania expression vector & $\begin{array}{l}\text { (Papadopoulou } \\
\text { et al., 1992; } \\
\text { Gourbal et al., } \\
\text { 2004) }\end{array}$ \\
\hline pSP72 aneo $\alpha$ & Leishmania expression vector & $\begin{array}{l}\text { (Papadopoulou } \\
\text { et al., 1992; } \\
\text { Gourbal et al., } \\
\text { 2004) }\end{array}$ \\
\hline $\begin{array}{l}\text { AQP1wT- } \\
\text { pSP72 } 2 \text { hygroa }\end{array}$ & $\begin{array}{l}\text { L. major wild type AQP } 1 \text { gene cloned into the } \\
\text { XbaI-HindIII sites of pSP } 72 \alpha \text { hygro } \alpha\end{array}$ & $\begin{array}{l}\text { (Figarella et al., } \\
\text { 2007) }\end{array}$ \\
\hline $\begin{array}{l}\text { AQP1к12A- } \\
\text { pSP72 } \alpha \text { hygro } \alpha\end{array}$ & $\begin{array}{l}\text { Site directed mutagenesis of the Lys12 codon to } \\
\text { an alanine codon in the AQP1 gene of AQP1wT } \\
\text {-pSP72 } \alpha \text { hygro } \alpha\end{array}$ & This study \\
\hline $\begin{array}{l}\text { AQP1K12R- } \\
\text { pSP72 } 2 \text { hygro } \alpha\end{array}$ & $\begin{array}{l}\text { Site directed mutagenesis of the Lys } 12 \text { codon to } \\
\text { an arginine codon in the AQP1 gene of } \\
\text { AQP1 } 1 \text { wT -pSP72 } \alpha \text { hygro } \alpha\end{array}$ & This study \\
\hline $\begin{array}{l}\text { MPK2wT- } \\
\text { pSP72 } \alpha \text { neo } \alpha\end{array}$ & $\begin{array}{l}\text { L. major wild type MPK2 gene cloned into the } \\
\text { XbaI-HindIII sites of pSP } 72 \alpha \text { neo } \alpha\end{array}$ & $\begin{array}{l}\text { (Mandal et al., } \\
\text { 2012) }\end{array}$ \\
\hline $\begin{array}{l}\text { MPK2K42A- } \\
\text { pSP72 } \alpha \text { neo } \alpha\end{array}$ & $\begin{array}{l}\text { Site directed mutagenesis of the Lys } 42 \text { codon } \\
\text { to an alanine codon in the MPK } 2 \text { gene of } \\
\text { MPK } 2 \text { wT -pSP72aneo } \alpha\end{array}$ & $\begin{array}{l}\text { (Mandal et al., } \\
\text { 2012) }\end{array}$ \\
\hline
\end{tabular}

\section{Strains}

Leishmania

LdBOB L. donovani wild type strain

(Goyard et al., 2003)

Vector alone

LdBob co-transfected with pSP72 $\alpha$ hygro $\alpha$ and This study pSP72 $\alpha$ neo $\alpha$

AQP1wT LdBob co-transfected with AQP1wT-

This study pSP72 $\alpha$ hygro $\alpha$ and pSP72 aneo $\alpha$ 


\begin{tabular}{|c|c|c|}
\hline $\mathrm{AQP} 1_{\mathrm{K} 12 \mathrm{~A}}$ & $\begin{array}{l}\text { LdBob co-transfected with AQP1K12A- } \\
\text { pSP72 } \alpha \text { hygro } \alpha \text { and pSP72 } \alpha \text { neo } \alpha\end{array}$ & This study \\
\hline $\mathrm{AQP} 1_{\mathrm{K} 12 \mathrm{R}}$ & $\begin{array}{l}\text { LdBob co-transfected with AQP1K12R- } \\
\text { pSP72 } \alpha \text { hygro } \alpha \text { and pSP72 } \alpha \text { neo } \alpha\end{array}$ & This study \\
\hline MPK2 wT & $\begin{array}{l}\text { LdBob co-transfected with MPK2 wT- } \\
\text { pSP72 } \alpha \text { neo } \alpha \text { and pSP72 } \alpha \text { hygro } \alpha\end{array}$ & This study \\
\hline MPK2К К42A & $\begin{array}{l}\text { LdBob co-transfected with MPK2K42A- } \\
\text { pSP72 } \alpha \text { neo } \alpha \text { and pSP72 } \alpha \text { hygro } \alpha\end{array}$ & This study \\
\hline $\begin{array}{l}\text { AQP1wT }+ \\
\text { MPK2wT }\end{array}$ & $\begin{array}{l}\text { LdBob co-transfected with AQP1wT- } \\
\text { pSP72 } \alpha \text { hygro } \alpha \text { and MPK2 wT-pSP72 } \alpha \text { neo } \alpha\end{array}$ & This study \\
\hline $\begin{array}{l}\mathrm{AQP} 1 \mathrm{~K} 12 \mathrm{~A}+ \\
\mathrm{MPK} 2 \mathrm{WT}\end{array}$ & $\begin{array}{l}\text { LdBob co-transfected with AQP1K12A- } \\
\text { pSP72 } \alpha \text { hygro } \alpha \text { and MPK2 wT-pSP72 } \alpha \text { neo } \alpha\end{array}$ & This study \\
\hline $\begin{array}{l}\mathrm{AQP} 1 \mathrm{~K} 12 \mathrm{R}+ \\
\mathrm{MPK} 2 \mathrm{WT}\end{array}$ & $\begin{array}{l}\text { LdBob co-transfected with AQP1K12R- } \\
\text { pSP72 } \alpha \text { hygro } \alpha \text { and MPK2 wT-pSP72 } \alpha \text { neo } \alpha\end{array}$ & This study \\
\hline $\begin{array}{l}\mathrm{AQP} 1 \mathrm{wT}+ \\
\text { MPK2 } 242 \mathrm{~A}\end{array}$ & $\begin{array}{l}\text { LdBob co-transfected with AQP1 wT- } \\
\text { pSP72 } \alpha \text { hygro } \alpha \text { and MPK2 K42A-pSP72 } \alpha \text { neo } \alpha\end{array}$ & This study \\
\hline $\begin{array}{l}\mathrm{AQP} 1 \mathrm{~K} 12 \mathrm{~A}+ \\
\mathrm{MPK} 2{ }_{\mathrm{K}} 42 \mathrm{~A}\end{array}$ & $\begin{array}{l}\text { LdBob co-transfected with AQP1K12A- } \\
\text { pSP72 } \alpha \text { hygro } \alpha \text { and MPK2 K42A-pSP72 } \alpha \text { neo } \alpha\end{array}$ & This study \\
\hline $\begin{array}{l}\mathrm{AQP} 1_{\mathrm{K} 12 \mathrm{R}}+ \\
\mathrm{MPK} 2_{\mathrm{K} 42 \mathrm{~A}}\end{array}$ & $\begin{array}{l}\text { LdBob co-transfected with AQP1K12R- } \\
\text { pSP72 } \alpha \text { hygro } \alpha \text { and MPK2 K42A-pSP72 } \alpha \text { neo } \alpha\end{array}$ & This study \\
\hline
\end{tabular}

\subsubsection{Oligonucleotide-directed mutagenesis}

Wild type Leishmania AQP1 gene was cloned in pGEMT-Easy vector as described previously (Gourbal et al., 2004). Alterations were created using the Quickchange site directed mutagenesis kit (Stratagene). In this process, supercoiled double-stranded DNA(dsDNA) vector with a gene of interest was used as a template. Two oligonucleotide primers containing the desired mutation were used, each complementary to the opposite strands of the vector. Pfu Turbo DNA polymerase used in the polymerase chain reaction (PCR) replicates both plasmid strands without 
displacement of the mutagenic oligonucleotide primers. Integration of mutated primers generates a mutated plasmid containing staggered nicks. Then, the PCR product was treated with $D p n$ I, an endonuclease enzyme specific for methylated and hemimethylated DNA. This enzyme is particularly used for the digestion of parental DNA template and for the selection of newly synthesized DNA containing mutation. The nicked, circular dsDNA was transformed in XL1-Blue supercompetent cells (Agilent), which helps in the repair of nicks in the mutated plasmid (Figure 16). 
Step 1

Plasmid Preparation

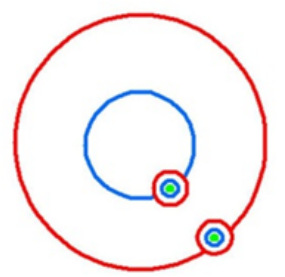

\section{Step 2}

Temperature Cycling

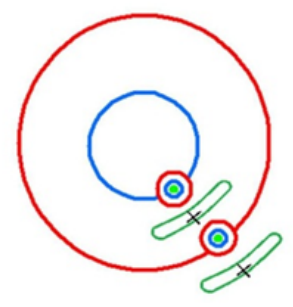

Mutagenic

primers

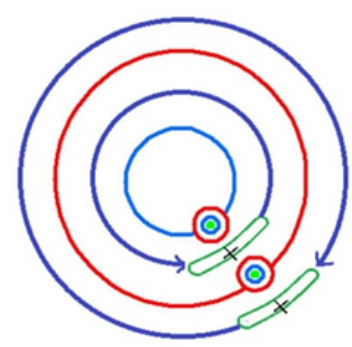

Step 3

Digestion

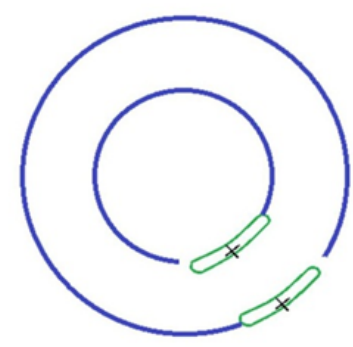

Step 4

Transformation

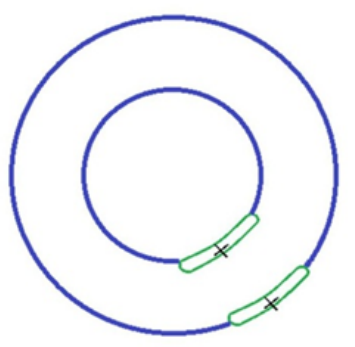

Mutated plasmid

(contains nicked circular strands)

Figure 16. Overview of the QuikChange site directed mutagenesis method (Agilent) 
The putative ubiquitination site lysine 12 (K12) of AQP1 was altered to alanine (A) or arginine (R). The mutagenic oligonucleotides used for both strands and the respective changes introduced (underlined) are as follows: K12A, 5'GAGGACCAACACGAGAGTGCGCGAAACTTCATGTCGCAG-3' (sense) and 5'CTGCGACATGAAGTTTCGCGCACTCTCGTGTTGGTCCTC-3’ (antisense); K12R, 5'- GACCAACACGAGAGTAGGCGAAACTTCATGTCG-3' (sense) and 5'CGACATGAAGTTTCGCCTACTCTCGTGTTGGTC-3' (antisense). Each mutation was confirmed by sequencing the entire gene (Eton biosciences, North Carolina, USA). Altered AQP1 was digested with HindIII and Xba I and cloned into the same sites of pSP72 $\alpha$ hygroa. Altered Leishmania mitogen activated protein kinase 2 (MPK2) used in this study was described previously (Mandal et al., 2012). Briefly, Leishmania major MPK2 was PCR amplified from genomic promastigote DNA and the PCR product was gel purified. The resulting fragment was cloned into pGEM-T Easy vector (Promega), and sequenced. The MPK2/pGEM-T Easy construct was subcloned into the yeast expression vector pYES2 (Life Technologies) followed by digestion with HindIII and XbaI and cloned into the same sites of pSP72- $\alpha$ neo $\alpha$ giving rise to MPK2-pSP72 $\alpha$ neo $\alpha$ (Mandal et al., 2012).

\subsubsection{Transfection of AQP1 and MPK2 in Leishmania}

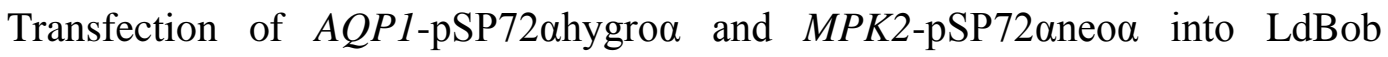
promastigotes were accomplished as described previously (Mandal et al., 2012). Briefly, $5 \mathrm{ml}$ of stationary phase promastigote cells were centrifuged at $3000 \mathrm{rpm}$ for $5 \mathrm{~min}$ at room temperature. Cells were washed once with HEPES-NaCl buffer. Cell pellet was 
resuspended in HEPES-NaCl buffer. $2 \times 10^{8}$ cells/ml were used for transfection of $2 \mu \mathrm{g}$ DNA. Cells containing DNA were electroporated in a cuvette $(0.4 \mathrm{~cm}$, Biorad $)$ using Gene Pulsar (Biorad). Cells were immediately pipetted into T25 flask (Corning) containing $5 \mathrm{ml}$ of M199 medium. All altered MPK2 and AQP1 constructs were transfected one at a time for each strain created for this study. All transfectants were maintained in the presence of $30 \mathrm{mg} \mathrm{ml}^{-1}$ hygromycin B and $60 \mathrm{mg} \mathrm{ml}^{-1}$ geneticin.

\subsubsection{Antimony sensitivity assay}

Antimony sensitivity profiles of the promastigotes were carried out as described previously (Mandal et al., 2012). Briefly, $1 \times 10^{7} \log$ phase cells were diluted 10 fold in culture medium (M199) containing increasing concentration of $\mathrm{Sb}(\mathrm{III})$ in the form of potassium antimonyl tartrate. Cell growth was monitored after $72 \mathrm{~h}$ at $\mathrm{A}_{600}$ using a microplate reader (Spectramax 340, Molecular Devices). EC50 was calculated from the linear regression curve of \% viability of cell at each concentration of $\mathrm{Sb}(\mathrm{III})$. Each assay was performed at least three times in triplicates. Errors bars were calculated from the mean \pm SE (Sharma et al., 2015).

\subsubsection{Uptake assay}

Uptake assay was performed using log phase promastigotes. Cells were washed twice with PBS, pH 7.4 (Thermo scientific) and resuspended in PBS at a density of $10^{8}$ cells $\mathrm{ml}^{-1}$. Cells were incubated with $10 \mu \mathrm{M}$ potassium antimonyl tartarate [Sb(III)] for $10 \mathrm{~min}$ at room temperature. A $200 \mu \mathrm{l}$ of cell suspension was filtered through nitrocellulose membrane and the membrane was washed with 5ml of ice-cold PBS. The 
membranes were digested with $0.4 \mathrm{ml}$ of concentrated $\mathrm{HNO}_{3}$ (69-70\%; EM Science) for one hour at $70^{\circ} \mathrm{C}$, allowed to cool to room temperature, and diluted with high-pressure liquid chromatography grade water (Sigma) to produce a final concentration of approximately 3\% $\mathrm{HNO}_{3}$. Samples were analyzed by PerkinElmer SCIEX ELAN® DRC-e inductively coupled plasma mass spectrometer. Standard solutions were made in the range of $0.5-10 \mathrm{ppb}$ in $3 \% \mathrm{HNO}_{3}$ using antimony standards (Ultra Scientific). Each transport experiment was repeated at least three times with duplicate samples. Error bars were calculated from the mean \pm SE. ( Sharma et al., 2015).

\subsubsection{Cell volume measurements}

Swelling is the first response of any animal cell exposed to a hypo-osmotic stress. Swollen cells start to expel several electrolytes and amino acids via active transporters to create a hyperosmotic zone near the vicinity of the cell membrane. This hyperosmotic zone drags the water out from the cells exclusively through aquaporins allowing the cells to regain their cell volume. This phenomenon is known as regulatory volume decrease (RVD) (Figure 17).

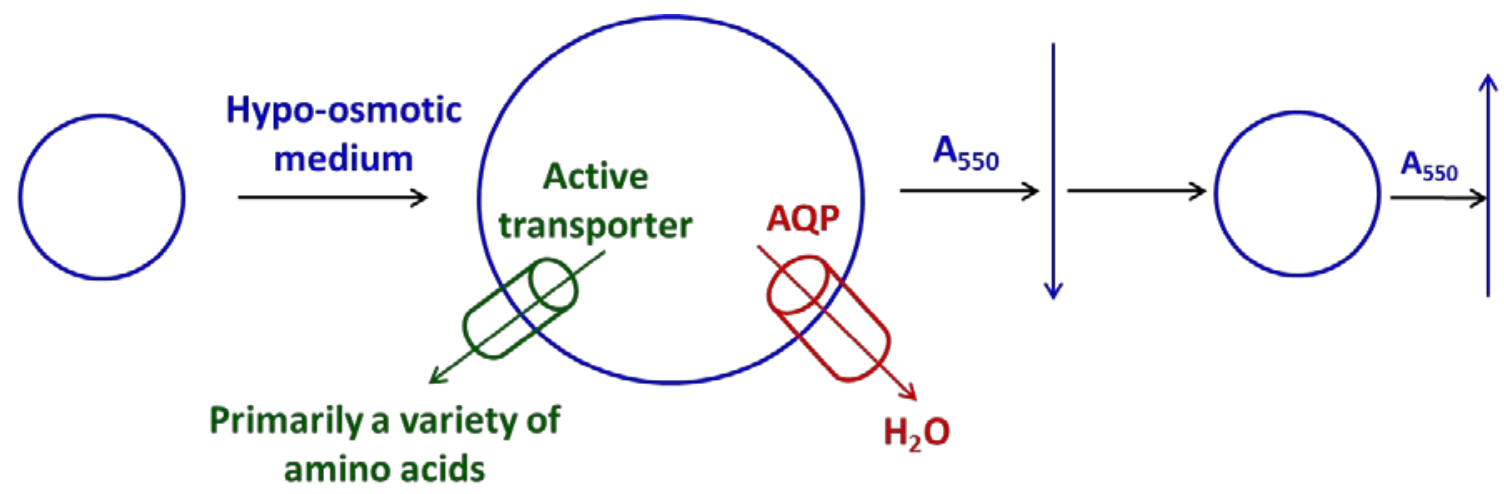

Figure 17. Principle of Regulatory Volume Decrease (RVD) phenomenon 
Mukhopadhyay's lab have shown that rate of RVD is directly proportional to the level of LmAQP1 expression in a Leishmania (Figarella et al., 2007). Relative changes in cell volume were measured upon introduction of hypo-osmotic stress as described previously (Mandal et al., 2012). Briefly, log phase promastigotes were washed twice with PBS and resuspended at a density of $10^{9}$ cells $\mathrm{ml}^{-1}$. A $100 \mu \mathrm{l}$ of cells were transferred to the microtiter plate and hypo-osmotic shock was induced by dilution of the isotonic cell suspension with an equal volume of deionized water and the absorbance at $550 \mathrm{~nm}$ was recorded every $15 \mathrm{sec}$ for $3 \mathrm{~min}$ in a microplate reader (Spectramax 340, Molecular Devices). A decrease in absorbance corresponds to an increase in cell volume and vice versa. Isosmotic control experiments consisted of dilution of cell suspensions with appropriate volumes of isosmotic buffer. All hypo-osmotic shock experiments were conducted at a final osmolarity of 150 mOsm (1:1 dilution of isosmotic buffer and water). Experiments were repeated for three times in triplicate. Error bars were calculated from the mean \pm SE.

\subsubsection{Flagella and pellicular membrane isolation and Immunoblotting}

The flagellar and pellicular plasma membrane fractions from LdBob wild type promastigotes or transfected with vector alone, altered $A Q P 1$ and $M P K 2$ were isolated as described previously (Mandal et al., 2012). All procedures were performed at $4^{\circ} \mathrm{C}$. Briefly, flagellar membrane fractions were isolated using $150 \mathrm{ml}$ of 3 days old promastigote culture. Cell were washed twice with ice-cold Buffer A (25 mM Tris-HCl, pH 7.4, $0.2 \mathrm{mM}$ EDTA, $5 \mathrm{mM} \mathrm{MgCl2}, 12 \mathrm{mM} \beta$-mercaptoethanol, and 0.32 M sucrose) and resuspended in $3 \mathrm{ml}$ of ice-cold sonication buffer [Buffer A, 1\% BSA, $0.1 \mathrm{mM} \mathrm{CaCl}_{2}$ 
and Complete Protease Inhibitor Cocktail (Roche)]. Flagella were detached without lysing the body of the cells by sonication on ice using three pulses of 1 s each, with $25 \%$ amplitude. Flagellar preparation was isolated from the supernatant by centrifuging at 700 $\mathrm{g}$ for $15 \mathrm{~min}$ followed by pelleting at $6800 \mathrm{~g}$ for $20 \mathrm{~min}$. The pellet was resuspended in Buffer B (25 mM Tris-HCl, pH 7.4, 0.2 mM EDTA, $5 \mathrm{mM} \mathrm{MgCl2,} 12 \mathrm{mM} \beta$ mercaptoethanol) and loaded onto $0.8 \mathrm{M}$ sucrose cushion and centrifuged at $1080 \mathrm{~g}$ for $20 \mathrm{~min}$. The upper layer was removed and overlaid on a discontinuous gradient of sucrose $(1.65 \mathrm{M}$ and $1.85 \mathrm{M})$ and ultracentrifuged at $133000 \mathrm{~g}$ for $3 \mathrm{~h}$. The interphase flagellar layer was removed, resuspended in Buffer A and ultracentrifuged at $143000 \mathrm{~g}$ for 45 min. Depending on the size, pellet was resuspended in $60-80 \mu 1$ of Buffer A. Pellicular membrane fractions were isolated as follows. 3 days old promastigote cells (150 ml culture) were harvested. Cells were washed with Buffer C (75 mM Tris-HCl, pH 7.4, $140 \mathrm{mM} \mathrm{NaCl}, 11 \mathrm{mM} \mathrm{KCl}$ ), then resuspended in Buffer D [10 mM HEPES, pH 7.4, $10 \mathrm{mM} \mathrm{KCl}, 1 \mathrm{mM} \mathrm{MgCl}$, $400 \mathrm{mM}$ mannitol, and Complete Protease Inhibitor Cocktail (Roche)] and broken by sonication using five pulses of $10 \mathrm{~s}$ each with $50 \%$ amplitude. Unbroken cells and cell debris were removed by centrifugation at $3000 \mathrm{~g}$ for $10 \mathrm{~min}$. The supernatant was centrifuged at $17000 \mathrm{~g}$ for $40 \mathrm{~min}$ to remove organelles and other intracellular membranes followed by centrifugation at $140000 \mathrm{~g}$ for $1 \mathrm{~h}$. The pellet, highly enriched in plasma membrane fraction, was washed and suspended in $1 \mathrm{ml}$ of Buffer E (75 mM HEPES, pH 7.4, $150 \mathrm{mM} \mathrm{KCl,} 2 \mathrm{mM} \mathrm{MgCl}$ ). Protein contents of either the pellicular membrane enriched or flagellar fractions were determined by the BCA protein assay kit (Pierce). The total protein contents of either the flagellar or pellicular plasma membrane fractions were determined by a filter assay method 
(Schaffner and Weissmann 1973). For immunoblot analysis, $25 \mu \mathrm{g}$ of flagellar protein samples and $45 \mu \mathrm{g}$ of pellicular protein samples were electrophoresed into a $12 \%$ SDSpolyacrylamide gel, and electro-blotted onto nitrocellulose membrane (Whatman). The blot was incubated with affinity-purified AQP1 antiserum as described previously (Figarella et al., 2007). The labelling was visualized with horseradish peroxidaseconjugated goat anti-rabbit serum (Sigma) using a Western Lightning Chemiluminescence Reagent Plus system (PerkinElmer).

Rabbit polyclonal anti-peptide antibody (anti-LmxMPK2) was used to detect MPK2 expression in Leishmania. For analysis of LdBob lysates, $\sim 2 \mathrm{X} 10^{7}$ cells were boiled in $100 \mu \mathrm{l}$ of lysis buffer [1X PBS, $0.1 \%$ (w/v) SDS, $50 \mathrm{mM}$ DDT, $50 \mu \mathrm{M}$ leupeptin, $\quad 25 \mu \mu \mathrm{M} \quad \mathrm{N} \alpha-p$-Tosyl-L-lysine chloromethyl ketone, $\quad 1 \quad \mathrm{mM}$ phenylmethanesulfonyl fluoride, $10 \mathrm{mM} \mathrm{1,10-phenanthroline,} \mathrm{and} \mathrm{1X} \mathrm{Laemmli} \mathrm{sample}$ buffer]. Twenty microliter of each sample were subjected to 12\% SDS-PAGE and electro-blotted onto nitrocellulose membrane. The blots were incubated with the antiLmxMPK2 antibody (1:100 dilution) overnight at $4^{\circ} \mathrm{C}$. The labeling was visualized with horseradish peroxidase-conjugated goat anti-rabbit serum (Sigma) using a Western Lightning Chemiluminescence Reagent Plus system (PerkinElmer) (Mandal et al., 2012)

\subsubsection{Treatment with proteosome inhibitor and immunoblot}

Promastigotes over expressing AQP1wT or co-overexpressing and AQP1wT + MPК2к42A were treated with the $10 \mu \mathrm{M}$ proteasome inhibitor MG132 and incubated for 48 hrs. Total plasma membrane protein was isolated as described above for pellicular membranes. The total protein content of the membranes was determined using filter 
assay method (Schaffner and Weissmann 1973). For immunoblot analysis, $50 \mu \mathrm{g}$ of total membrane protein sample was electrophoresed into a 12\% SDS-polyacrylamide gel, and electroblotted onto nitrocellulose membrane (Whatman). The blot was incubated with affinity-purified AQP1 antiserum as described previously (Figarella et al., 2007). The labeling was visualized with horseradish peroxidase-conjugated goat anti-rabbit serum (Sigma) using a Western Lightning Chemiluminescence Reagent Plus system (PerkinElmer). Cells treated with MG132 were also examined for antimonite sensitivity and $\mathrm{Sb}(\mathrm{III})$ accumulation as described above.

For determining EC50, MG132 treated cells for $48 \mathrm{~h}$ were washed, diluted to $2 \mathrm{x}$ $10^{6}$ cells $/ \mathrm{ml}$ in fresh medium with MG132 and, treated with different concentrations of potassium antimonyl tartrate. EC50 was determined by counting cells manually in a hemocytometer. For Sb(III) uptake MG132 treated cells were washed once with PBS+10 $\mu \mathrm{M}$ MG132, and resuspended in PBS at a density of $10^{8}$ cells $\mathrm{ml}^{-1}$. Sb(III) accumulation in whole cells was measured as described above in section 2.2.5.

\subsubsection{Immunofluorescence analysis}

Late-log-phase LdBob promastigotes $\left(5 \mathrm{X} 10^{5}\right.$ cells) were fixed in $4 \%$ paraformaldehyde/PBS overnight at $4^{0} \mathrm{C}$. The cells were washed twice with PBS, transferred to teflon coated immunofluorescence slides (Polysciences), and permeabilized with $0.1 \%$ TritonX-100, $50 \mathrm{mM} \mathrm{Na} 2 \mathrm{PO}_{4}, 50 \mathrm{mM}$ glycine, $\mathrm{pH}$ 7.2, for 2 min. After blocking with $1 \%$ bovine serum albumin (BSA) in PBS for 30 min, cells were incubated for $1 \mathrm{~h}$ at room temperature with AQP1 anti-serum (1:300 in 1\% BSA). The primary antibody was removed by washing three times with $1 \%$ BSA followed by incubation with 
the monoclonal anti-tubulin antibody (Sigma-Aldrich) 1:500 for another hour. After three washes with 1\% BSA, the slides were incubated with Alexa Fluor ${ }^{\circledR 488-c o n j u g a t e d ~}$ goat anti-rabbit IgG (Life Technologies) (1:1000 in 1\% BSA) and Alexa Fluor®594conjugated goat anti-mouse IgG (Life Technologies) (1:2000 in 1\% BSA) for $1 \mathrm{~h}$. Finally, the cell nuclei were stained with DAPI (0.1 mg ml-1final concentration) (Sigma-Aldrich) for 10 min, the slides were washed three times with 1\% BSA, and once with sterile water. The slides were mounted in Vectashield®Antifade Mounting Medium (Vector Laboratories). The fluorescence labeled cells was examined by Elite Delta Vision microscope (GE) at 60X oil immersion lens.

\subsubsection{Determination of wild type and altered AQP1 half-lives from different transfectants}

Mid-log phase LdBob promastigotes were treated with $70 \mathrm{mg} \mathrm{ml}^{-1}$ cycloheximide and cells were harvested at different time points as described previously (Mandal et al., 2012). Flagella were isolated from cells collected at each time-point. Levels of AQP1 expression were analyzed as described above. However, for AQP1 ${ }_{\mathrm{wT}}+\mathrm{MPK} 2 \mathrm{~K} 42 \mathrm{~A}$ a

total flagellar protein of $70 \mu \mathrm{g}$ was loaded in each lane. Densitometric analyses of expression of $A Q P 1$ were performed from immunoblots using the ImageJ software (http://imagej.nih.gov/ij/). The expression ratio was determined after normalizing AQP1 band intensity at each time-point against that at zero hour.

\subsubsection{Determination of AQP1 mRNA levels from different transfectants}

Total RNA was isolated from each transfectant and AQP1 mRNA levels from different transfectants were determined by QPCR as described previously (Mandal et al., 
2015). $2 \mu \mathrm{l}$ of diluted purified cDNA reaction corresponding to $6 \mathrm{ng}$ of template RNA, were used in a $10 \mu \mathrm{l}$ reaction containing forward (5'-CTGTGTCTTTGGTGCCTTTCC3') and reverse (5'-GCCTTTTGGGCGTCGTC-3') primers for AQP1. The relative abundance of target amplicons between samples was estimated using glyceraldehyde 3phosphate dehydrogenase (GAPDH) (forward primer 5'GGAGATAGACAAGGCCATCAAG-3' and reverse primer 5'TGTCGTTGATGAAGTCAGAGC-3') as loading control by the $2^{-\triangle \Delta C T}$ method (Livak and Schmittgen 2001). Error bars were calculated from the mean \pm SD of three independent experiments in triplicate.

\subsection{Results}

\subsubsection{AQP1 degradation is mediated by the proteasome}

Previously, we showed that AQP1wT was highly destabilized in $L$. donovani promastigotes when it was overexpressed in the presence of MPK2 $42 \mathrm{~A}$ that was unable to complete the phosphoryl transfer to its substrate. We asked whether this enhanced degradation below the steady state level is due to ubiquitination mediated leishmanial 26S proteasomal degradation. A wild type strain of L. donovani was chosen for the following reasons although the AQP1 knockout strain of $L$ major recently became available from the Ouellette lab (in collaboration with the Mukhopadhyay lab) (Plourdea 2015) : (i) this study is a logical extension of our previous findings performed in $L$. donovani, (ii) We have recently shown that $L$. donovani has the lowest level of AQP1 mRNA, is highly resistant to $\mathrm{Sb}(\mathrm{III}) /(\mathrm{V})$ and shows the lowest accumulation of $\mathrm{Sb}(\mathrm{III})$. It is also a very poor osmoregulator (Mandal et al., 2015). These properties of the LdBOB 
strain that we have used are almost as good as an AQP1 knockdown strain of Leishmania.

L. donovani was chosen in the absence of an AQP1 knockout strain, as this species has the lowest level of AQP1 mRNA level, is highly resistant to $\mathrm{Sb}(\mathrm{III}) /(\mathrm{V})$ and shows the lowest accumulation of $\mathrm{Sb}(\mathrm{III})$. It is also a very poor osmoregulator (Mandal et al., 2015). L. donovani promastigotes overexpressing AQP1wt alone and AQP1wT + MPK2к42A were treated with $10 \mu \mathrm{M}$ MG132, a global proteasome inhibitor, for $48 \mathrm{hr}$. We could not isolate flagella from these cells as they became circular with very short flagella because of metabolic stress (Figure 18).
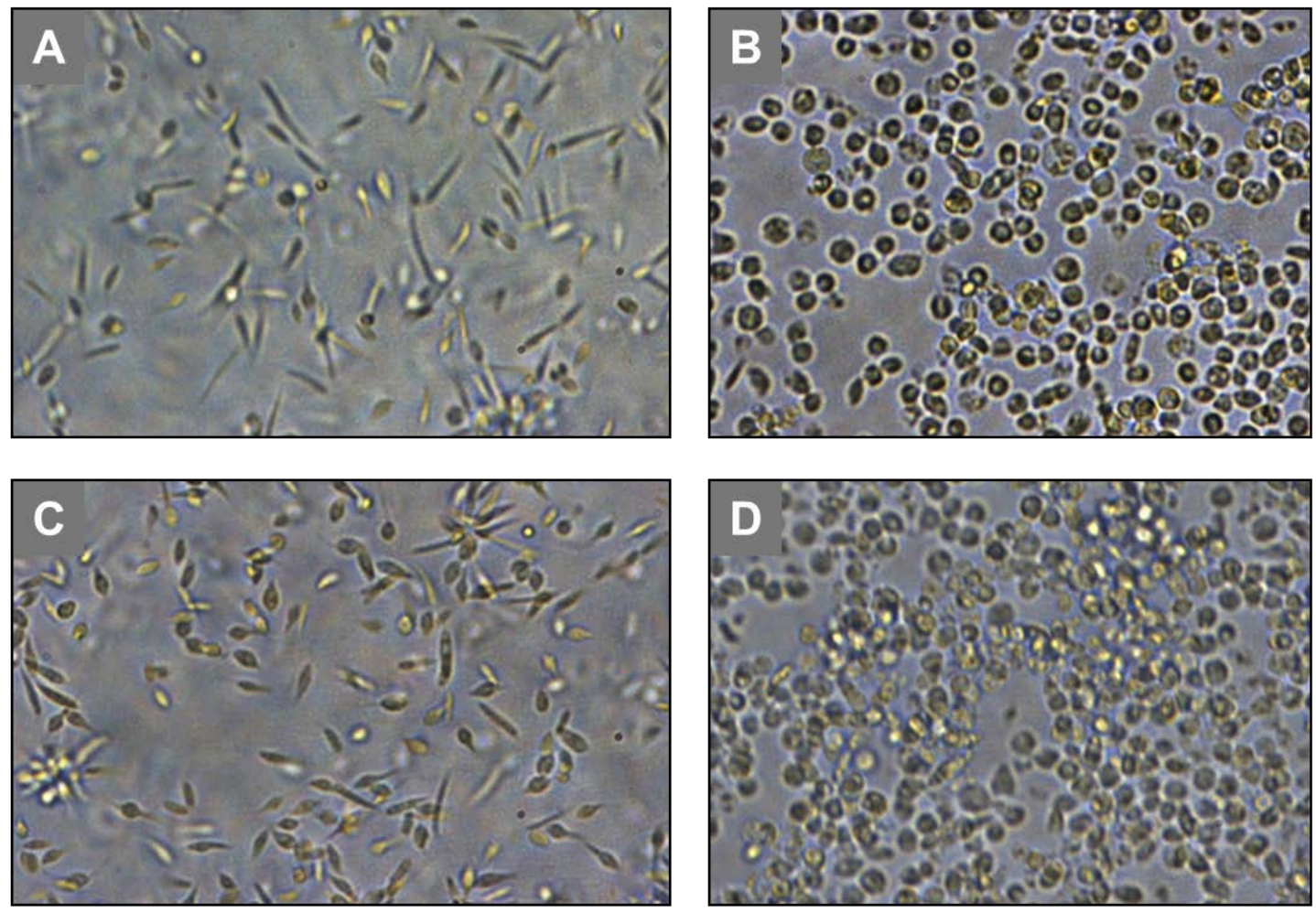

Figure 18. Morphological changes in $\mathrm{LdBOB}$ promastigotes upon treatment with MG132. LdBOB promastigotes expressing (A) AQP1 $1_{\mathrm{wT}}$ (untreated); (B) AQP1 $1_{\mathrm{WT}}$

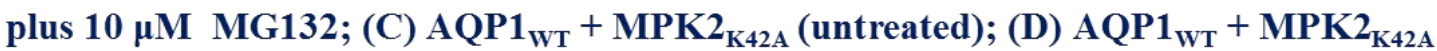
plus $10 \mu \mathrm{M}$ MG132. 
Cell shape change due to MG132 has been observed before in mammalian cells (Ukekawa et al., 2004; Li et al., 2013). A lower concentration (5 $\mu \mathrm{M}$ ) of MG132, which was reported previously (Perez-Pertejo et al., 2011), also elicited a similar change in cell shape. In addition, MG132 slowed down the growth of the promastigotes. However, cell viability was not compromised. Therefore, total membrane protein was isolated from these cells and AQP1 expression was observed by immunoblot. Previously, work from the Mukhopadhyay lab observed that AQP1 was not detected in total plasma membranes isolated from the promastigotes even when overexpressing AQP1wT (Figarella et al., 2007). However, total plasma membrane isolated from promastigotes treated with MG132 showed expression of AQP1 even when the protein was completely destabilized in AQP1wT + MPK2K42A in the absence of MG132 (Figure 19A). This shows the protective behavior of a proteasome inhibitor on AQP1. The MG132 treated promastigotes were also hypersensitive to $\mathrm{Sb}(\mathrm{III})$ and accumulated more of the metalloid when compared to untreated cells (Figure 19B). AQP1wT cells treated with MG132 were three times more sensitive to $\mathrm{Sb}(\mathrm{III})$ and accumulated twice as much of the drug when compared to cells grown in the absence of MG132 (Figure 19C). AQP1 WT $+\mathrm{MPK}_{\mathrm{K} 42 \mathrm{~A}}$ cells became ten times more hypersensitive to Sb(III) (Figure 19B) and accumulated four times as much of the antimonial drug (Figure 19C) when compared to the untreated promastigotes. 


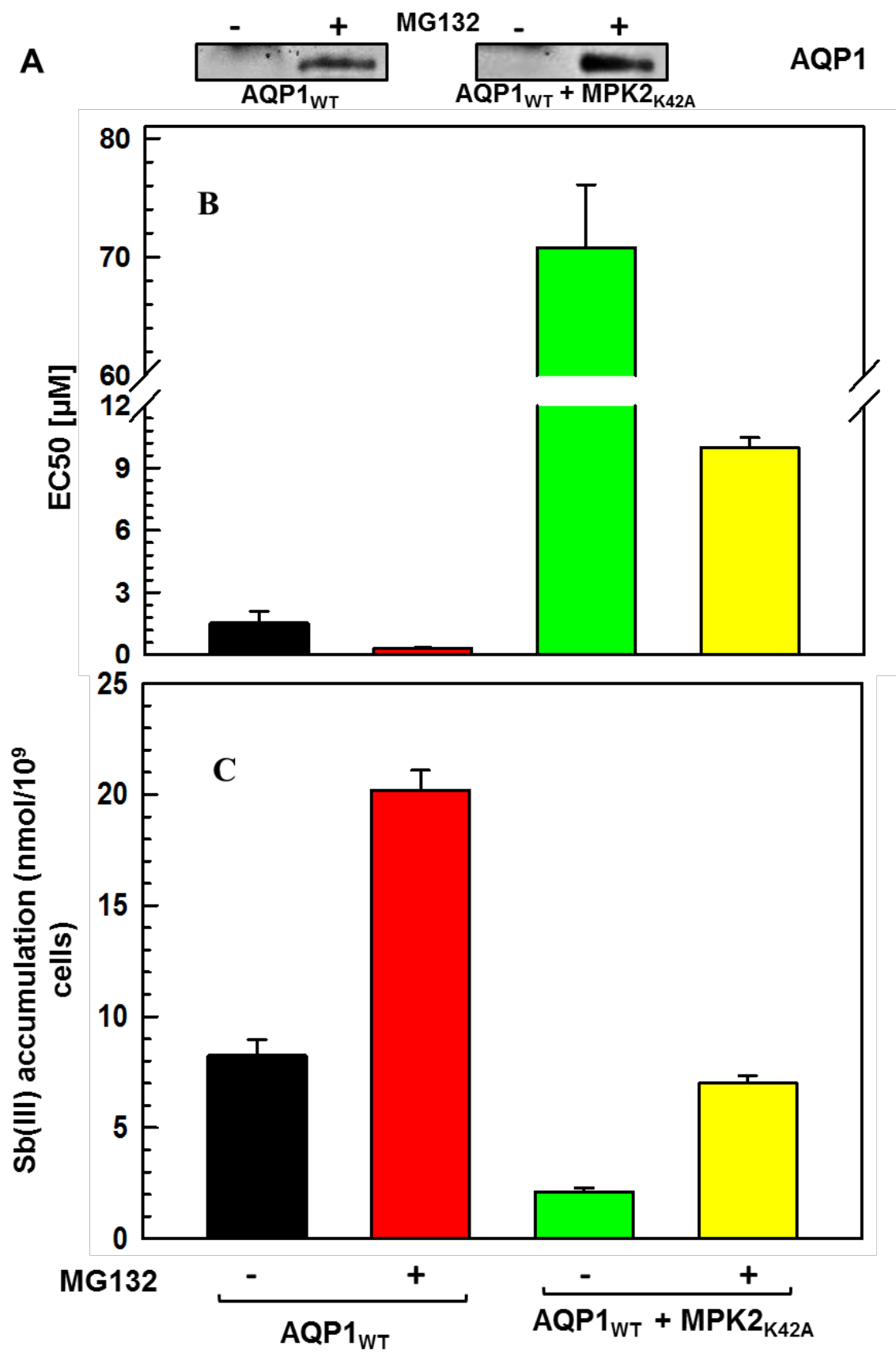

Figure 19. AQP1 is subjected to proteasome-mediated degradation. (A) LdBob transfectants were treated or not with the proteasome inhibitor MG132. Total plasma membrane fractions were then tested for the presence of AQP1 by Western blotting using an anti-AQP1 antibody; (B) Sb(III) sensitivity $\left(\mathrm{EC}_{50}\right)$ of LdBob transfectants \pm MG132; (C) Sb(III) uptake in LdBob transfectants \pm MG132. 


\subsubsection{Lysine 12 as the putative ubiquitination site of $\mathrm{AQP1}$}

Proteasome mediated degradation and/or endocytosis of membrane proteins including aquaporins takes place by ubiquitination at critical lysines exposed to the cytosol (Kamsteeg et al., 2006; Moeller et al., 2011). We analyzed the L. major AQP1 sequence by predicting a topology of AQP1 and by Ub-Pred (Radivojac et al., 2010).

Figure 20 shows that both $\mathrm{N}$-and C-termini of AQP1 are cytosolic.

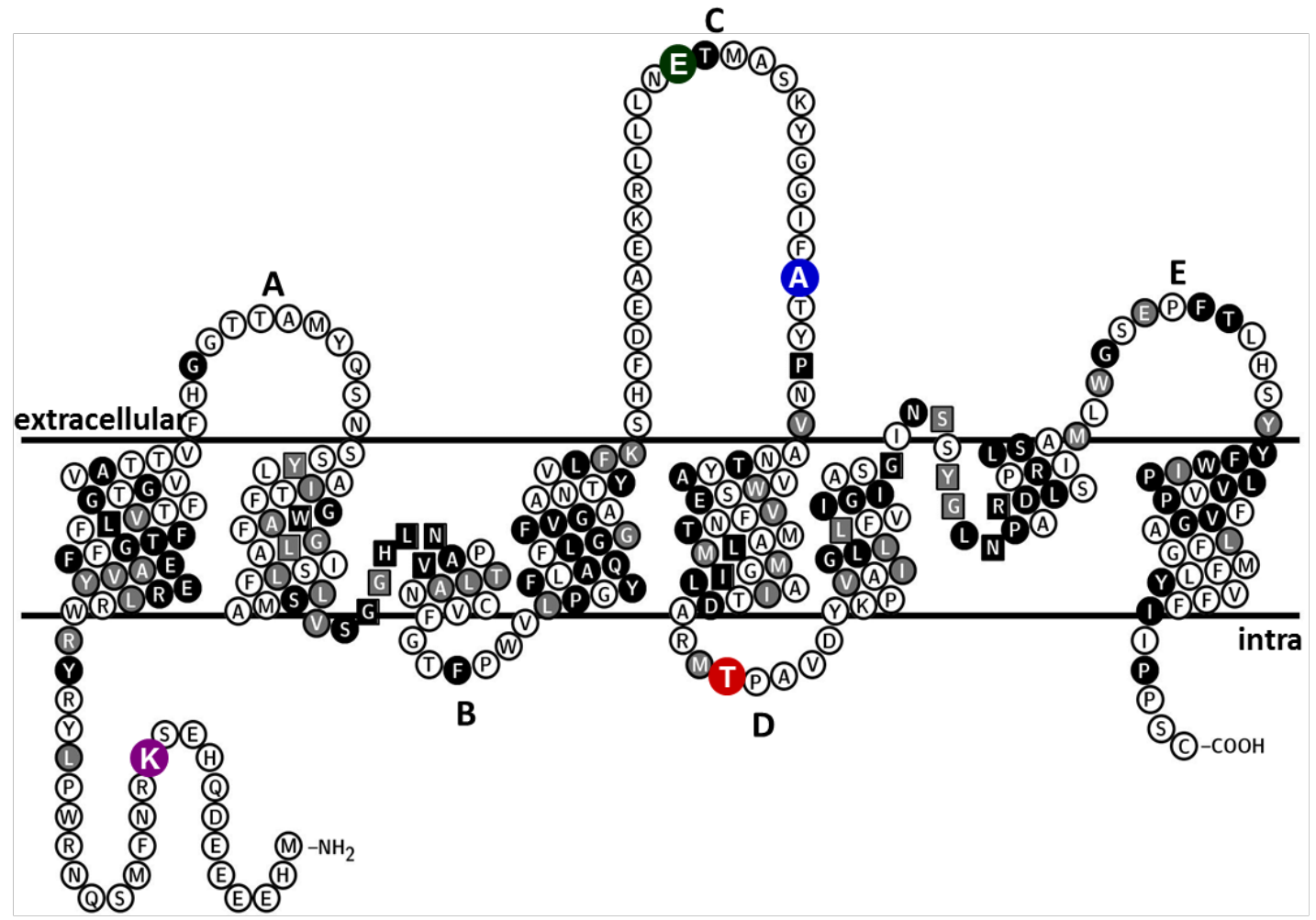

Figure 20. Predicted topology of L. major AQP1. The topology is based on the crystal structure of PfAQP (Newby et al., 2008) and plotted using the TeXtopo package (Beitz, 2000). Amino acids labeled as white letters in black circles are conserved between AQP1 and PfAQP. Residues marked as white letters in grey circles are similar to PfAQP; black and grey squares indicate conserved and similar residues, respectively, which directly interact with glycerol in PfAQP. Lys12, Glu152, Ala163 and Thr197 are labeled as white letters in colored circles. The five loops are indicated as $\mathbf{A}$ through $\mathbf{E}$. 
Figure 21 shows that lysine 12 (K12) is the only predicted ubiquitination site in AQP1 (http:/www.ubpred.org). $\mathrm{K} 12$ is also conserved across six major Leishmania species (Figure 22).

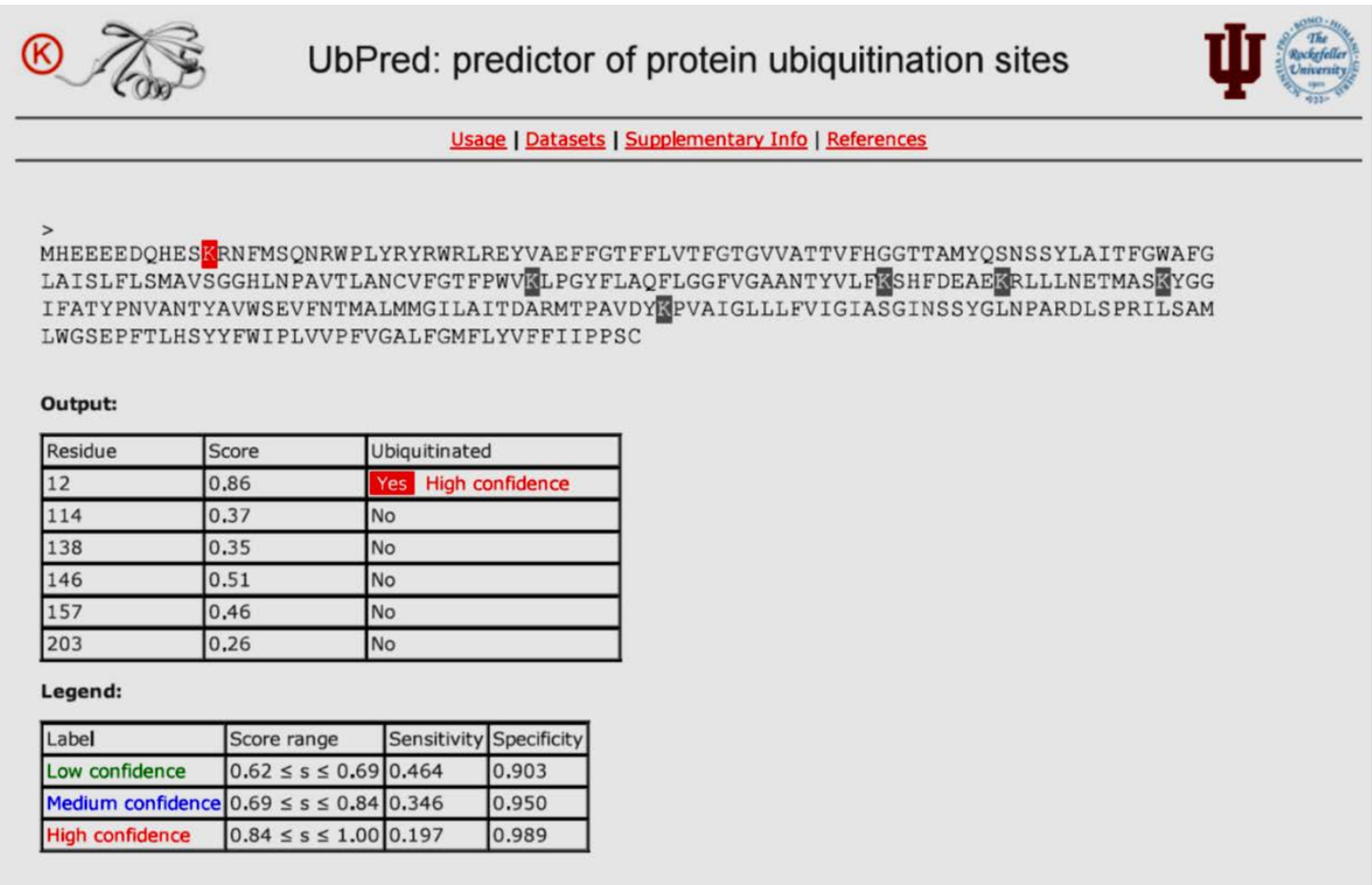

Figure 21. AQP1 sequence analysis by Ub-Pred 
K12

L. braziliensis

L. panamensis

L. major

L. tropica

L. donovani

L. infantum

L. braziliensis

L. major

L. tropica

L. donovani
L. panamensis

L. infantum

1 - - MDEDQQQRQKRDLTAODTWPLYRYRWWTREYVAEFFGSFFLLSFGIGVTATTTFHAGN 1 - - MDEDQQQRQKRDLTAQDTWPLYRYRWWIREYVAEFFGSFFLLSFGIGVTATTTFHAGN 1 MHE EEDQHESKRNFMSQNRWPLYRYRWR LREYVAEFFG TFFLVTFGTGVVATTVFHGGT 1 MHE EEGQLENKRNFTLQNRWPLYKYRWRIREYVAEFFGTFFLVTFGTGVIATTVFHAGN 1 MHE EQGQLEGKRNFTS QNRWPLYKYRWWLREYVAEFFG TFFLVTFGTGVIATTVFHAGN 1 MHEEQGQLEGKRNFTSQNRWPLYKYRWWLREYVAEFFG TFFLVTFGTGVIATTVFHAGN

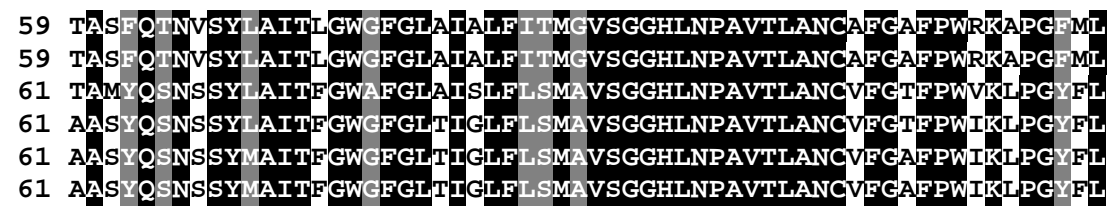

L. braziliensis

L. panamensis

L. major

L. tropica

L. donovani

$L$. infantum

L. braziliensis

L. panamensis

L. major

L. tropica

L. donovani

L. infantum

L. braziliensis

$L$. panamensis

L. major

L. tropica

L. donovani

L. infantum

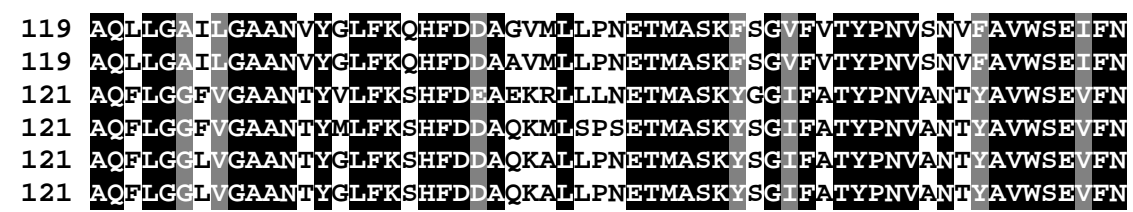

179 TMVLMMGILAINDNRMTPADGYKPVAVGLLLFVIGITTGINSGYALNPTRDLGPRIFTA 179 TMVLMMGILAINDNRMTPADGYKPVAVGLLLFVIGITTGINSGYALNPTRDLGPRIFTA 181 TMALMMGILAITDARMTPAVDYKPVAIGLLLFVIGIASGINSSYGLNPARDLSPRILSAM 181 TMALMMGILAITDPRMTPAVNYKPVAIGLLLFVIGITSGINSSYGLNPARDLSPRILSAI 181 TMALMMGILAITDPRMTPAVNYKPVAIGLLLFVIGITSGINSSYGLNPARDLSPRILSAM 181 TMALMMGILAITDPRMTPAVNYKPVAIGLLLFVIGITTGINSSYGLNPARDLSPRILSAM

239 LWGKEPFTLHGYYFWIPIVGPIAGALLGMFLYVFCIIPSGA 239 LWGKEPFILHGYYFWIPIVGPIAGALLGMFLYVFCIIPSGA 241 LWGSEPFTLHSYYFWIPLVVPFVGALFGMFLYVFFIIPPSC 241 LWGSEPFTLYSHYFWIPLVAPFVGALLGMFLYVFFIIPPSC 241 LWGSEPFTLYSYYFWIPLVAPFVGALLGMFLYVFFIIPPNF 241 LWGSEPFTLYSYYFWIPLVAPFVGALLGMFLYVFFIIPPNF

Figure 22. Protein sequence alignment of Leishmania AQP1 from six different species

Additionally, the disordered cytosolic N-terminus is often involved in accelerated degradation of proteins by ubiquitination (Landre et al., 2013 ; Vittal et al., 2015). We used RONN (Regional Order Neural Network) v3.2 (Yang et al., 2005) and PrDOS (protein disorder prediction system) (Ishida and Kinoshita 2007) to predict the disorder in AQP1 sequence; both software programs identified the cytosolic N-terminus where K12 is located as the only reasonable disordered site of the protein (Figure 20 and Figure 23). 
Therefore, we altered K12 of AQP1 to alanine (AQP1 $\left.{ }_{\mathrm{K} 12 \mathrm{~A}}\right)$ and arginine (AQP1 $\left.1_{\mathrm{K} 12 \mathrm{R}}\right)$ by site directed mutagenesis and over expressed alone or in the presence of wild type MPK2 or MPK2 K42A in L. donovani.
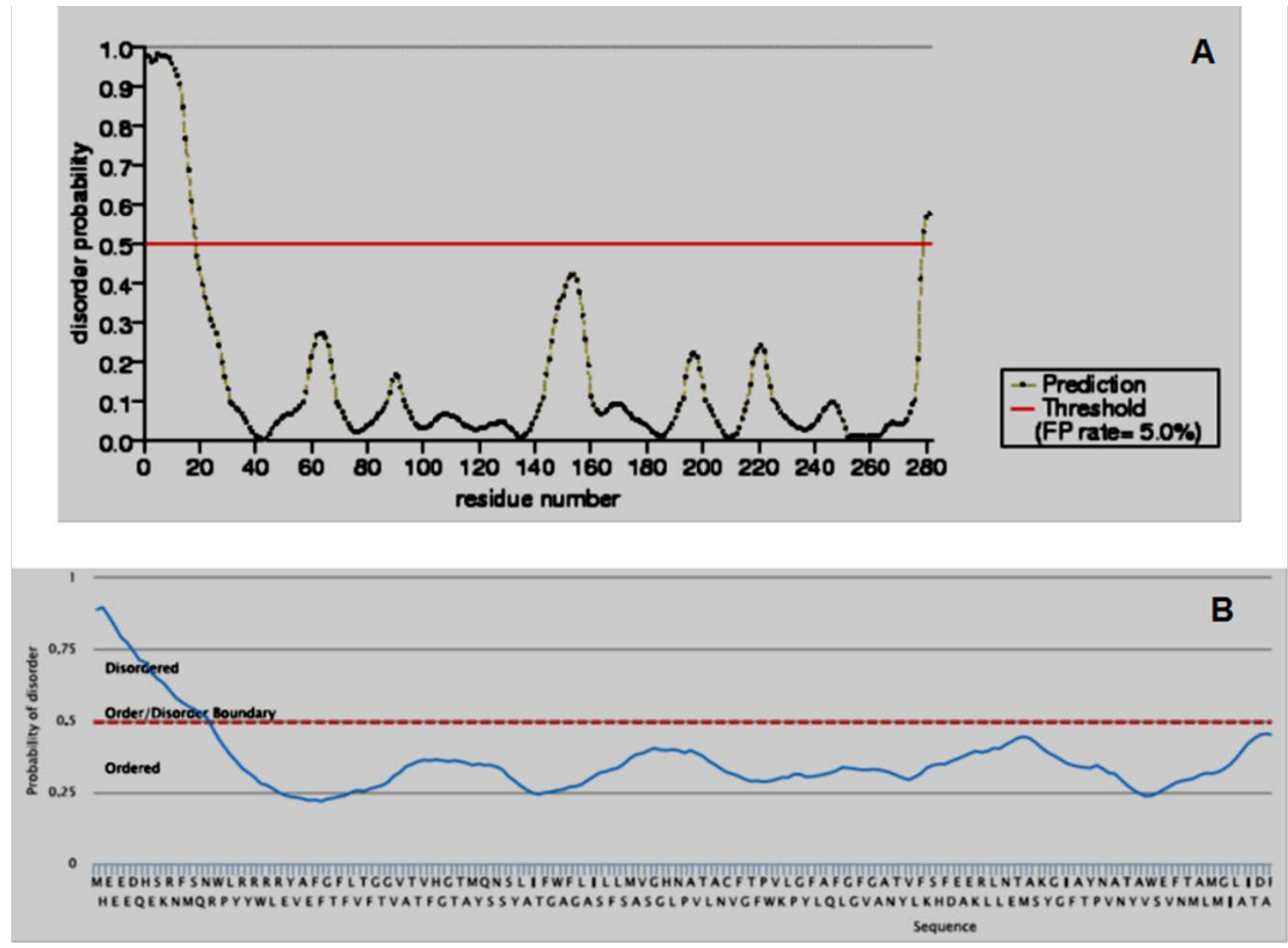

Figure 23. N-terminus of AQP1 is disordered. AQP1 sequence analysis by A. PrDOS and B. RONN v3.2 


\subsubsection{Alteration of lysine 12 of AQP1 confers efficient osmoregulation in Leishmania}

We have shown that the physiological function of AQP1 in Leishmania is osmoregulation. The strategic flagellar localization of AQP1 helps the parasite sense the changing osmotic environments between the vector and the host (Figarella et al., 2007). The Mukhopadhyay Lab previously showed that osmoregulatory capacity of the promastigotes was directly proportional to the AQP1 protein levels in the membrane (Figarella et al., 2007; Mandal et al., 2012; Mandal et al., 2015). Our work also demonstrated that when wild type AQP1 was co-expressed with MPK2 $442 \mathrm{~A}$, the antimony facilitator was destabilized and showed poor osmoregulation when compared to AQP1wT or AQP1 $\mathrm{wT}_{\mathrm{w}}+\mathrm{MPK} 2 \mathrm{wT}$ cells (Mandal et al., 2012) (for details of all strains/transfectants see Table 1). Cells expressing wild-type AQP1 exhibited a complete volume recovery in $56 \mathrm{~s}$ whereas the vector control needed $180 \mathrm{~s}$ (Figure 24 and Fig 25). Consistent with their slightly increased expression, cells expressing either AQP1K12A or AQP1к12R exhibited a complete volume recovery in $43 \mathrm{~s}$ and $30 \mathrm{~s}$, respectively (Figure 24 and Figure 25). When cells coexpressing wild-type AQP1 and MPK2 were subjected to a similar hypo-osmotic shock, the swelling and recovery process was essentially complete within the dead time of the spectrophotometer (Figure 24 and Figure 25). Cells coexpressing AQP1wT and MPK2 K42A exhibited near negligible expression of AQP1 and took nearly $150 \mathrm{~s}$ for volume recovery (Figure 24 and 25). In contrast, cells coexpressing $\mathrm{AQP} 1_{\mathrm{K} 12 \mathrm{~A}}$ or AQP1к12R and either the wild type or altered MPK2 exhibited an identical

volume recovery as cells transfected with wild type AQP1 and MPK2 (Figure 24 and Figure 25). Taken together, these results demonstrate that reduced AQP1 ubiquitination following alteration of Lys12 leads to increased protein stability and increased levels of 
AQP1 expression (Figure 28), which provides improved osmoregulation in promastigotes (Figure 24 and Figure 25).

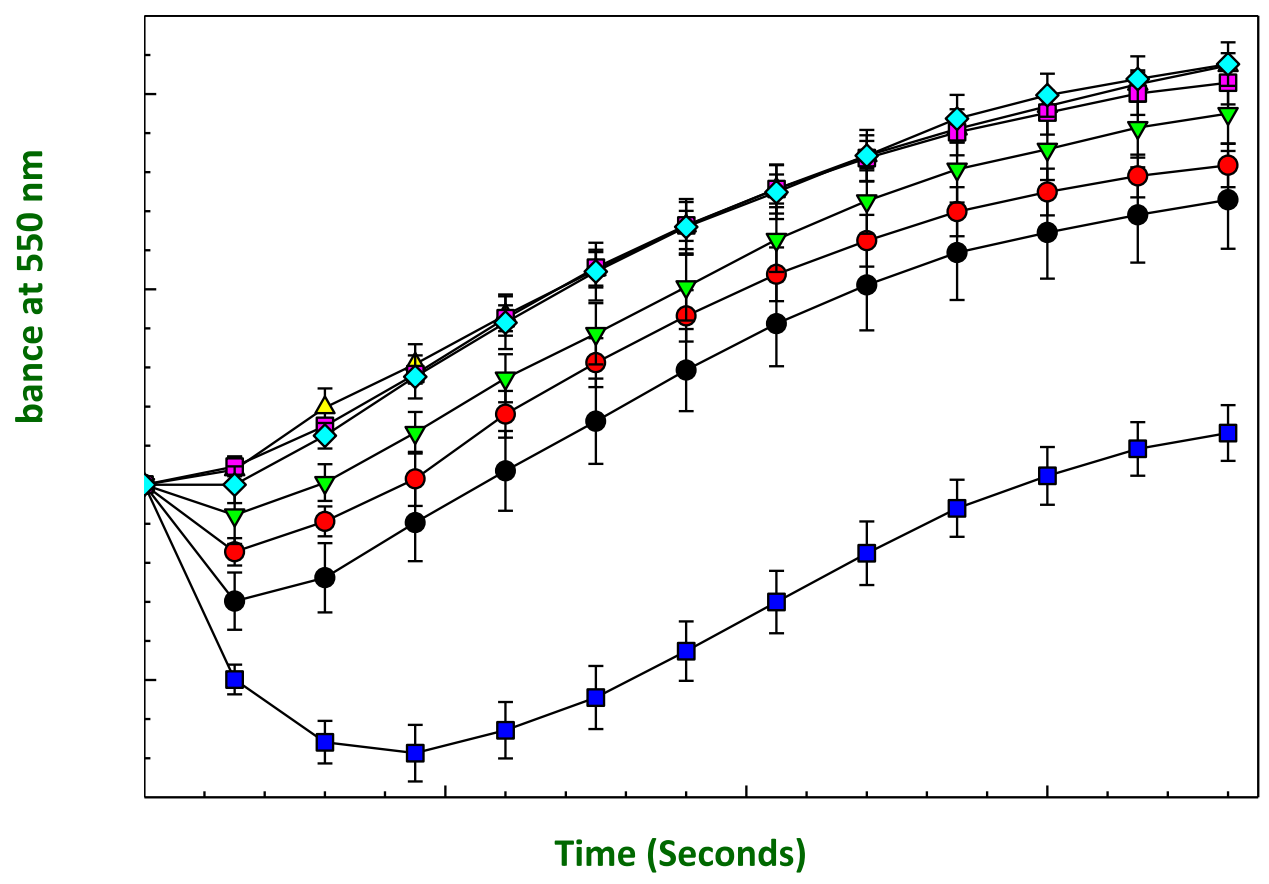




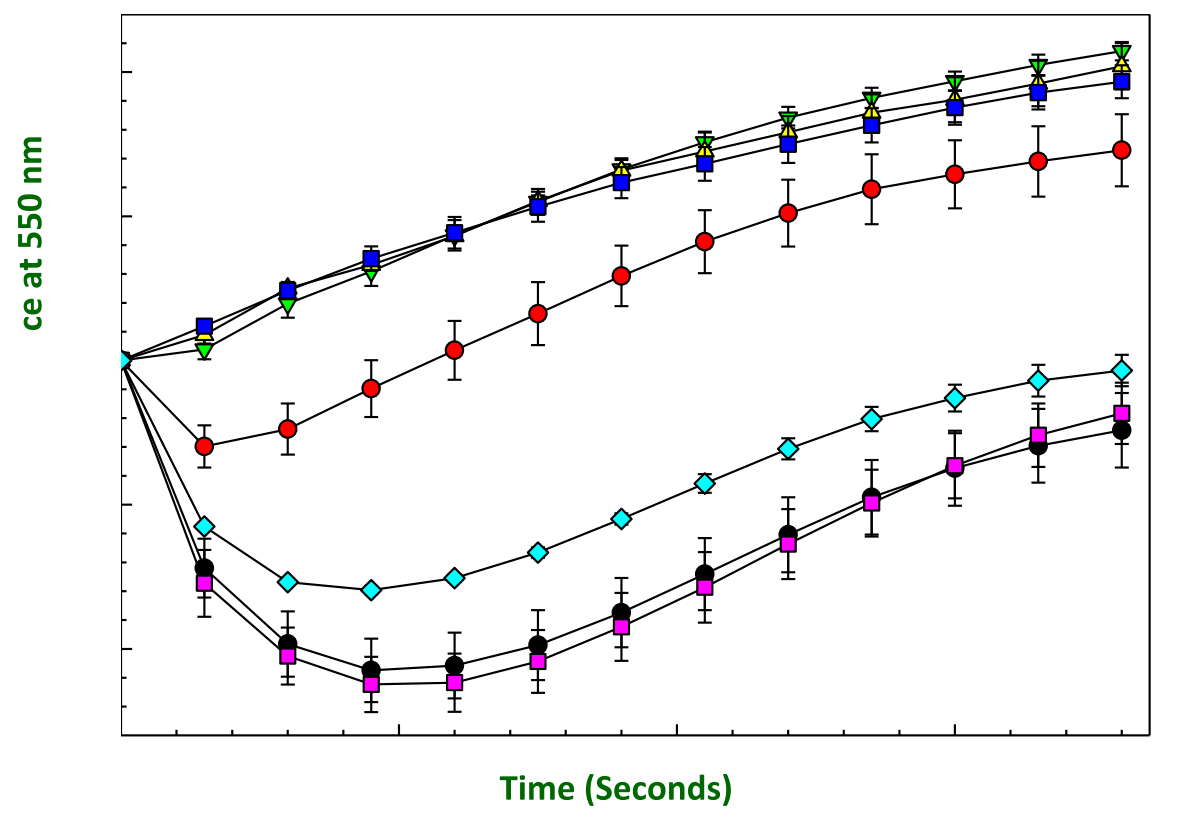

\subsubsection{Alteration of lysine 12 of AQP1 confers antimonite sensitivity in Leishmania}

Difference in $\mathrm{Sb}(\mathrm{III})$ sensitivity among the strains described above was determined by exposing the promastigotes at increasing concentrations of potassium antimonyl tartrate. AQP1 $\mathrm{wT}^{+}+\mathrm{MPK} 2 \mathrm{~K}_{42 \mathrm{~A}}$ cells were 80 times more resistant to $\mathrm{Sb}(\mathrm{III})$, whereas AQP1к12R and AQP1к12A cells were 1.5-2 times more sensitive to Sb(III) when compared to AQP1wт alone cells. However, when lysine 12 was replaced with alanine or arginine in AQP1 and co-expressed with MPK2 $442 \mathrm{~A}$, promastigotes became hypersensitive to $\mathrm{Sb}(\mathrm{III})$. $\mathrm{AQP} 1_{\mathrm{K} 12 \mathrm{~A}}+\mathrm{MPK} 2_{\mathrm{K} 42 \mathrm{~A}}$ and $\mathrm{AQP} 1_{\mathrm{K} 12 \mathrm{R}}+\mathrm{MPK} 2_{\mathrm{K} 42 \mathrm{~A}}$ cells were 600 times and 400 times more sensitive to $\mathrm{Sb}(\mathrm{III})$ respectively when compared to AQP1 ${ }_{\mathrm{wT}}+\mathrm{MPK} 2_{\mathrm{K} 42 \mathrm{~A}}$ cells (Table 2). On the other hand, 
AQP1 $1_{12 A} / A Q P 1_{K 12 R}+M P K 2{ }_{\mathrm{wT}}$ cells showed a similar sensitivity profile when compared to AQP1wT+MPK2wT cells (Table 2).

Table 2. Antimonite accumulation and sensitivity of LdBob cells expressing AQP1 and/or MPK2

\begin{tabular}{|c|c|c|}
\hline Strain & $\begin{array}{c}\text { Sb(III) accumulation } \\
\text { (nmol/10 } 10^{9} \text { cells) }\end{array}$ & $\begin{array}{c}\mathrm{EC}_{50} \\
{[\mathrm{Sb}(\mathrm{III})] \mu \mathrm{M}}\end{array}$ \\
\hline Vector & $2 \pm 0.2$ & $130 \pm 5$ \\
\hline $\mathrm{AQP} 1_{\mathrm{WT}}$ & $9 \pm 0.6$ & $1 \pm 0.01$ \\
\hline $\mathrm{AQP} 1_{\mathrm{K} 12 \mathrm{~A}}$ & $11 \pm 0.4$ & $0.7 \pm 0.09$ \\
\hline $\mathrm{AQP} 1_{\mathrm{K} 12 \mathrm{R}}$ & $12 \pm 0.7$ & $0.5 \pm 0.02$ \\
\hline $\mathrm{AQP} 1_{\mathrm{WT}}+\mathrm{MPK} 2_{\mathrm{WT}}$ & $21 \pm 0.4$ & $0.1 \pm 0.002$ \\
\hline $\mathrm{AQP} 1_{\mathrm{WT}}+\mathrm{MPK} 2_{\mathrm{K} 42 \mathrm{~A}}$ & $3 \pm 0.3$ & $80 \pm 3$ \\
\hline $\mathrm{AQP} 1_{\mathrm{K} 12 \mathrm{~A}}+\mathrm{MPK} 2_{\mathrm{WT}}$ & $19 \pm 0.7$ & $0.1 \pm 0.002$ \\
\hline $\mathrm{AQP} 1_{\mathrm{K} 12 \mathrm{~A}}+\mathrm{MPK} 2_{\mathrm{K} 42 \mathrm{~A}}$ & $21 \pm 0.7$ & $0.1 \pm 0.002$ \\
\hline $\mathrm{AQP} 1_{\mathrm{K} 12 \mathrm{R}}+\mathrm{MPK} 2_{\mathrm{WT}}$ & $19 \pm 0.6$ & $0.1 \pm 0.002$ \\
\hline $\mathrm{AQP} 1_{\mathrm{K} 12 \mathrm{R}}+\mathrm{MPK} 2_{\mathrm{K} 42 \mathrm{~A}}$ & $19 \pm 0.9$ & $0.1 \pm 0.002$ \\
\hline MPK2 & $2 \pm 0.2$ & $105 \pm 5$ \\
\hline $\mathrm{MPK} 2_{\mathrm{K} 42 \mathrm{~A}}$ & $2 \pm 0.2$ & $105 \pm 5$ \\
\hline
\end{tabular}

The $\mathrm{Sb}(\mathrm{III})$ accumulation in these various cell types were also examined. AQP1 ${ }_{\mathrm{wT}}+\mathrm{MPK} 2_{\mathrm{K} 42 \mathrm{~A}}$ cells had the lowest accumulation of $\mathrm{Sb}(\mathrm{III})$, which was similar to vector alone control, whereas AQP1 alone cells had 3 times more intracellular antimonite levels (Table 2) . AQP1 $1_{\mathrm{K} 12 \mathrm{~A}} / \mathrm{AQP} 1_{\mathrm{K} 12 \mathrm{R}}$ cells accumulated similar levels of Sb(III) when compared to promastigotes overexpressing wild type AQP1 alone. However, AQP1к12A + MPK2к42A and AQP1к12R+MPK2к42A cells accumulated 6 and 5.5 times more $\mathrm{Sb}(\mathrm{III})$ when compared to $\mathrm{AQP} 1 \mathrm{wT}+\mathrm{MPK} 2_{\mathrm{K} 42 \mathrm{~A}}$ cells. On the other hand, AQP1 ${ }_{\mathrm{K} 12 \mathrm{~A}} / \mathrm{AQP} 1_{\mathrm{K} 12 \mathrm{R}}+\mathrm{MPK} 2 \mathrm{WT}$ cells showed a similar $\mathrm{Sb}(\mathrm{III})$ accumulation profile to AQP1 ${ }_{\text {wT }}+$ MPK2 2 wT cells (Table 2). 


\subsubsection{Lysine 12 is responsible for $\mathrm{MPK} 2_{\mathrm{K} 42 \mathrm{~A}}$ mediated degradation of $\mathrm{AQP} 1$}

We performed immunoblot analysis to determine whether the reversal of osmoregulatory capacity, the hypersensitivity to $\mathrm{Sb}(\mathrm{III})$, and the higher accumulation of $\mathrm{Sb}(\mathrm{III})$ of $\mathrm{AQP} 1{ }_{\mathrm{wT}}+\mathrm{MPK} 2 \mathrm{~K} 42 \mathrm{~A}$ cells, when wild type AQP1 was exchanged with $\mathrm{AQP} 1 \mathrm{~K} 12 \mathrm{~A} / \mathrm{AQP} 1 \mathrm{~K} 12 \mathrm{R}$, was due to altered expression of AQP1. To examine the expression of AQP1, both flagellar and pellicular membrane fractions were isolated from the promastigotes of the various strains. Immunoblot analysis of the flagellar fraction probed with anti-LmAQP1 antibody (Figarella et al., 2007) confirmed our previous observation that MPK2 stabilized AQP1 and redistributed to the pellicular membrane; however, the antimonite facilitator was destabilized in the presence of MPK2 $442 \mathrm{~A}$ (Figure 26) (Mandal et al., 2012). When the putative ubiquitination site K12 of AQP1 was replaced with alanine or arginine, the МРК2к42А mediated destabilization was reversed (Figure 26). In fact, AQP1 expression in these strains restored to the levels of $\mathrm{AQP} 1 \mathrm{wT}^{+\mathrm{MPK} 2 \mathrm{wT}}$ cells, however, they were expressed exclusively in the flagellar membrane (Figure 26). Promastigotes overexpressing AQP1K12A/AQP1К12R could be detected at slightly higher levels ( $\mathrm{P}=0.03$ and $\mathrm{P}<0.001$, respectively) than wild type in the flagellar membrane indicating that alteration of lysine12 to either alanine or arginine increased AQP1 expression (Figure 26). Additionally, when AQP1к12A/AQP1к12R was co-expressed with wild type MPK2, AQP1 did not show any change in expression when compared to AQP1 $\mathrm{wT}_{\mathrm{T}}+\mathrm{MPK} 2 \mathrm{wT}$ cells. They did not redistribute to the pellicular membrane (Figure 26). 


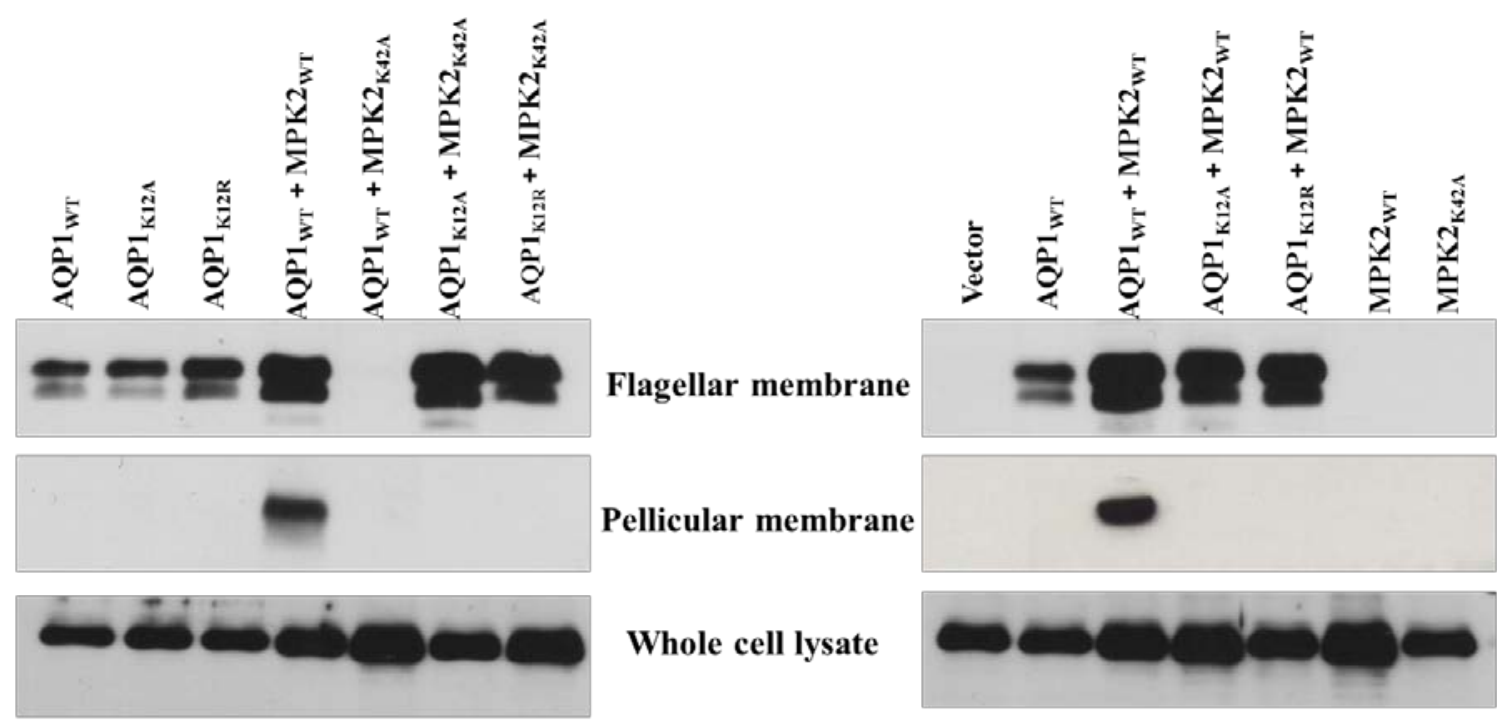

Figure 26. Cellular localization of AQP1. Immunoblot analysis of LdBob transfectants : flagellar and pellicular membrane fractions probed with anti-AQP1 antibody and whole cell lysate probed with anti-LmxMPK2 antibody

A polyclonal antibody (anti-LmxMPK2) against the synthetic peptide CGLESRPVEREAAVRK, corresponding to amino acids 444-458 of LmxMPK2 was used. Anti-LmxMPK2 can detect MPK2 as well as L. donovani MPK2 (LdoMPK2) (Mandal et al. 2012). Immunoblot analysis showed that MPK2 was expressed in promastigotes transfected with either MPK2 alone or co-transfected with AQP1 and MPK2. Expression of endogenous LdoMPK2 was also detected in cells transfected with either AQP1 or altered AQP1 or in the vector control. Cells expressing AQP1K12A/AQP1K12R co-transfected with MPK2 also showed a dense band similar to MPK2 (Figure 26).

Immunofluorescence experiments also supported the immunoblot data. As observed previously, AQP1 was detected in the flagellum of the promastigotes in AQP1 
alone cells (Figure 27A) and showed a diffused localization in AQP1 ${ }_{\mathrm{wT}}+\mathrm{MPK} 2 \mathrm{wT}$ cells (Figure 27B). AQP1 expression in the flagellum was hardly detected in AQP1 ${ }_{\mathrm{wT}}+\mathrm{MPK} 2 \mathrm{~K} 42 \mathrm{~A}$ cells (Figure 27C), whereas AQP1K12A/AQP1K12R $+\mathrm{MPK} 2 \mathrm{~K} 42 \mathrm{~A}$ cells exhibited an exclusive flagellar localization similar to AQP1 cells (Figure 27F and I). As expected, AQP1 ${ }_{\mathrm{K} 12 \mathrm{~A}} / \mathrm{AQP} 1_{\mathrm{K} 12 \mathrm{R}}$ alone (Figure $27 \mathrm{D}$ and $\mathrm{G}$ ) cells showed flagellar localization of AQP1. Interestingly, when coexpressed with MPK2wT, altered AQP1 was observed on the flagellum only but not on the pellicular membrane of promastigotes (Figure 27E and H). We interpret this observation as Lys12 being an essential element in the relocalization of AQP1 between different cellular compartments. In contrast to coexpression of wild type AQP1 and MPK2 ${ }_{\text {K42A }}$ where AQP1 is hardly detectable (Figure 27C), coexpression of altered AQP1with МРК2 $\mathrm{K}_{22} \mathrm{~A}$ showed the localization of $\mathrm{AQP} 1 \mathrm{~K} 12 \mathrm{~A}$ or AQP1K12R to flagellum alone (Figure 27F and I). These observations again suggest that alteration of Lys12 prevents proteasome-mediated degradation. 


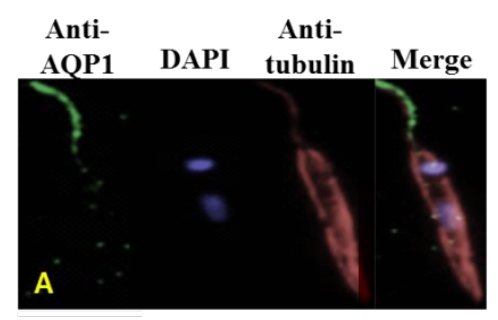

AQP1 $_{\text {wT }}$

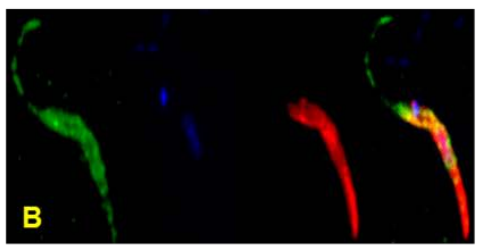

$A Q P 1_{\mathrm{wT}}+\mathrm{MPK2}_{\mathrm{wT}}$

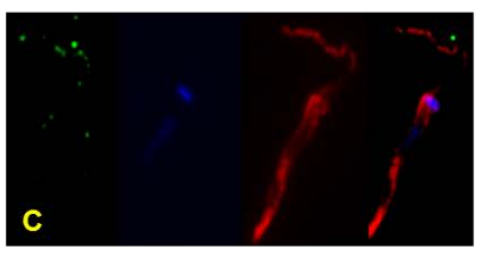

$A Q P 1_{\mathrm{WT}}+\mathrm{MPK}_{\mathrm{K} 42 \mathrm{~A}}$

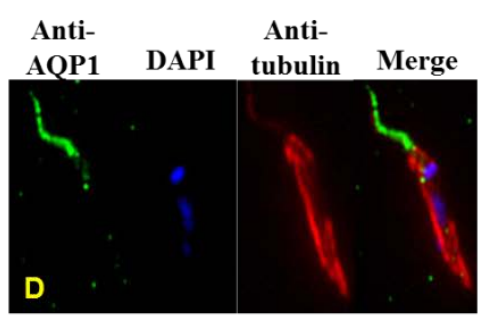

$\mathrm{AQP1}_{\mathrm{K} 12 \mathrm{~A}}$

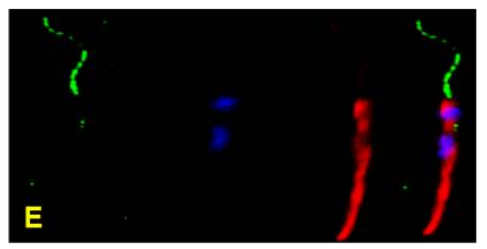

$\mathrm{AQP1}_{\mathrm{K} 12 \mathrm{~A}}+\mathrm{MPK2}_{\mathrm{WT}}$

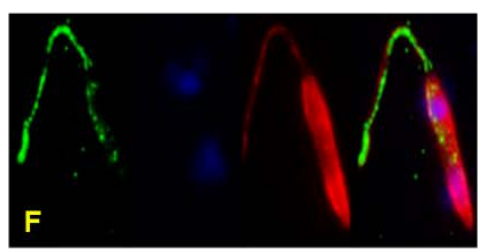

$A Q P 1_{\mathrm{K} 12 \mathrm{~A}}+\mathrm{MPK}_{\mathrm{K} 42 \mathrm{~A}}$

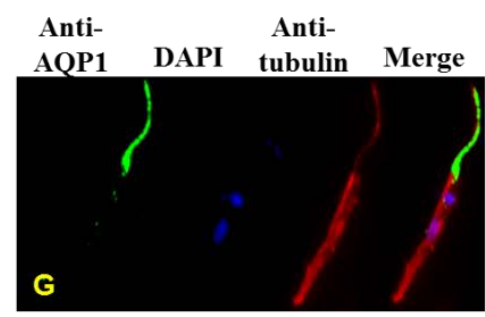

$A Q P 1_{K 12 R}$

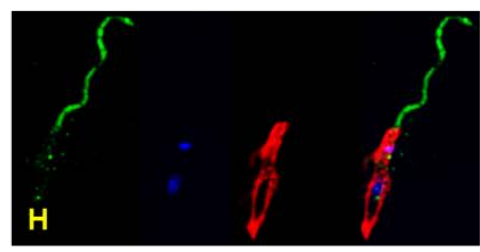

$\mathrm{AQP1}_{\mathrm{K}_{22 \mathrm{R}}}+\mathrm{MPK}_{\mathrm{WT}}$

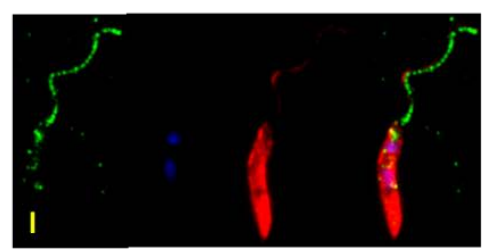

$A Q P 1_{\mathrm{K} 12 \mathrm{R}}+\mathrm{MPK}_{\mathrm{K} 42 \mathrm{~A}}$

Figure 27. Immunofluorescence microscopy of LdBob transfectants. Cells were stained for AQP1 (green), DNA (blue), and $\alpha$-tubulin (red). The three images were finally merged.

\subsubsection{Reversal of K42AMPK2 mediated destabilization of AQP1 by alteration of lysine 12 takes place by increasing its half life}

To determine whether removal of K12 stabilizes AQP1 in the presence of MPK2 $42 \mathrm{~A}$ by modification of its turnover rate, cycloheximide-blocking experiments were performed to measure protein half-lives. Mid log phase promastigotes were treated with cycloheximide, followed by isolation of flagella at defined time intervals, and the decay of AQP1 was determined by SDS-PAGE and immunoblot analysis (Figure 28). AQP1 from AQP1 ${ }_{w T}+$ MPK2 42 A cells was most unstable showing a half-life of only $<5$

h, whereas the $\mathrm{Sb}(\mathrm{III})$ facilitator from cells expressing AQP1 alone had a half-life of >60 h (Figure 28). AQP1 ${ }_{\mathrm{K} 12 \mathrm{~A}} / \mathrm{AQP} 1_{\mathrm{K} 12 \mathrm{R}}$ alone also had half-life similar to AQP1 alone. As 
observed before (Mandal et al., 2012), AQP1 from AQP1wT+MPK2wT cells were highly stable and showed only about 5\% degradation during the whole experiment. It was interesting to note that when this critical lysine was replaced with alanine, the MPK2к42A mediated degradation of AQP1 was stopped, and the half-life of the altered AQP1 was increased to the AQP1 $\mathrm{wT}^{+}+\mathrm{MPK} 2 \mathrm{wT}$ levels. Curiously, AQP1 from AQP1к12A/AQP1 $12{ }_{\mathrm{K}}+\mathrm{MPK} 2 \mathrm{~K} 42 \mathrm{~A}$ cells showed a little instability up to $12 \mathrm{~h}(20-40 \%)$ but remained stable for the rest of the time period of the experiment. These data clearly depict that removal of $\mathrm{K} 12$ at the N-terminus of AQP1 in the presence of MPK2 $442 \mathrm{~A}$ decreases the turnover number of AQP1.

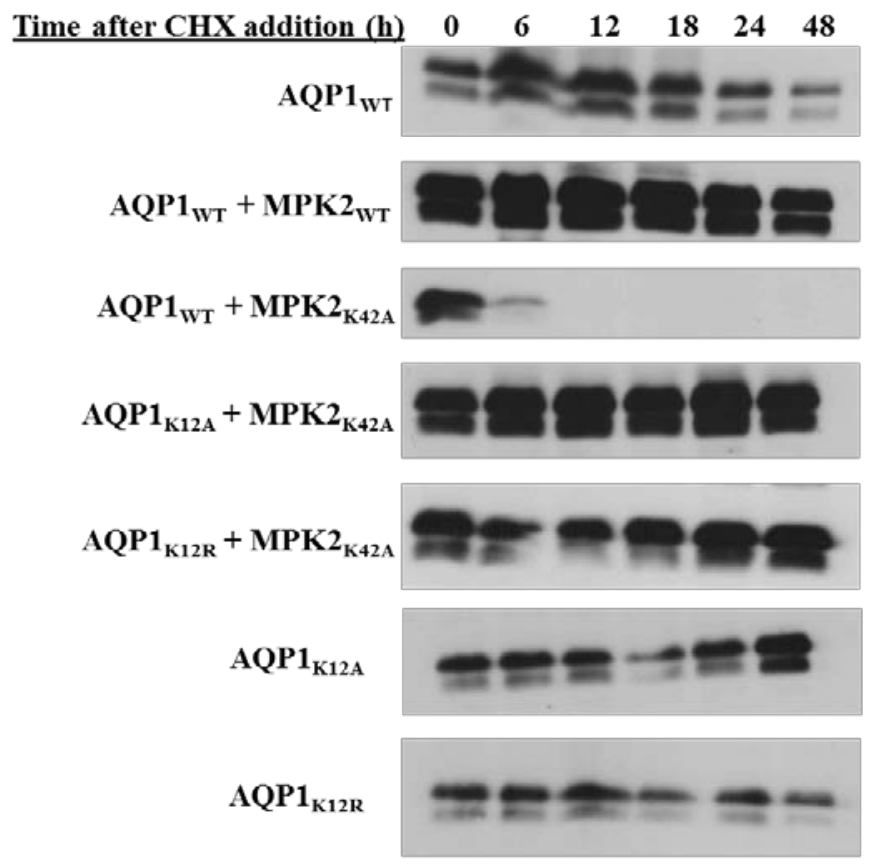

Figure 28. Turnover of AQP1. Mid-log phase promastigotes were treated with $70 \mu \mathrm{g} \mathrm{ml}^{-1}$ cycloheximide (CHX). Cells were harvested at the indicated times following $\mathrm{CHX}$ addition. Immunoblot analysis of the flagellar membrane fractions probed with anti-AQP1 antibody. 


\subsection{Discussion}

Aquaporin expression was stabilized from various cell types by proteasome inhibitors. For example, osmotic stress decreased AQP4 expression in a retinal epithelial cell line which was reversed by proteasomal inhibitors (Willermain et al., 2014). AQP1 expression in mammalian fibroblasts was increased after treatment with the proteasomal inhibitor MG132 (Leitch et al., 2001). AQP2 in mouse inner medullary collecting duct MCD3 cells was stabilized after treatment with MG132 (Umenishi et al., 2005). Therefore, ubiquitin-proteasome mediated degradation pathways of aquaporins have been described in various mammalian cell types, but not from kinetoplastids. Proteasome mediated degradation of proteins requires ubiquitination at specific lysine residues. However, the existence of ubiquitin mediated post-translational regulation of parasite aquaporins especially in Leishmania is unknown.

In this study, we show that Leishmania AQP1 was upregulated, and the promastigotes became hypersensitive when treated with a proteasomal inhibitor MG132 (Figure 19B). Accumulation of Sb(III) was also increased in MG132 treated cells (Figure 19C). We analyzed the AQP1 sequence using multiple software programs and identified K12 as the only putative ubiquitination site at the highly disordered N-terminus (Figure 21). Altering $\mathrm{K} 12$ to alanine/arginine of $\mathrm{AQP} 1$ and co-expressing it with $\mathrm{MPK} 2 \mathrm{~K} 42 \mathrm{~A}$ reversed its stability (Figure 26) by increasing its half-life (Figure 28). Removal of this critical lysine from the disordered $\mathrm{N}$-terminus of AQP1 also restored the antimonial sensitivity of the promastigotes because they accumulated more of the drug (Table 2). However, we have been unable to show a direct ubiquitination event at K12 by 
immunoprecipitation experiments. Pulling down ubiquitinated AQP1 either by anti-Ub or anti-AQP1 followed by an immunoblot failed because both of these polyclonal antibodies cross-reacted with each other in addition to their non-specific binding to other ubiquitinated proteins in the flagellar membrane. In spite of the fact that the immunoprecipitation experiments failed, our data strongly suggest K12 as the putative ubiquitination site in AQP1 that accelerates its degradation in presence of MPK2 K42A.

Earlier, we showed that AQP1, when phosphorylated in the presence of MPK2, was redistributed to the pellicular membrane along with the flagellar membrane (Mandal et al., 2012). We interpreted that AQP1 was stabilized because of a conformational change due to phosphorylation at T197 and that it was enough for a relocalization signal. However, our current data, presented here, indicated that AQP1 did not redistribute to the pellicular membrane in the absence/presence of phosphorylation as soon as it stabilized in the absence of the putative ubiquitination site K12 (Figure 26 and 27). AQP1 maintained its flagellar localization in AQP1 ${ }_{\mathrm{K} 12 \mathrm{~A}} / \mathrm{AQP} 1_{\mathrm{K} 12 \mathrm{R}}+\mathrm{MPK} 2 \mathrm{WT}$ or AQP1 ${ }_{\mathrm{K} 12 \mathrm{~A}} / \mathrm{AQP} 1_{\mathrm{K} 12 \mathrm{R}}+\mathrm{MPK}_{\mathrm{K} 42 \mathrm{~A}}$ promastigotes, in spite of the fact that it was highly stabilized with a very low turnover number. No changes in the mRNA levels of AQP1 were observed in the strains where AQP1 was highly stabilized when compared to AQP1 alone or AQP1wT+MPK2K42A cells (Table 3). 


\section{Table 3. AQP1 mRNA levels in various transfectants}

\begin{tabular}{lc}
\hline $\mathrm{AQP} 1_{\mathrm{WT}}$ & 1 \\
$\mathrm{AQP} 1_{\mathrm{K} 12 \mathrm{R}}$ & $0.85 \pm 0.28$ \\
$\mathrm{AQP} 1_{\mathrm{K} 12 \mathrm{~A}}$ & $1.24 \pm 0.12$ \\
$\mathrm{AQP} 1_{\mathrm{WT}}+\mathrm{MPK} 2_{\mathrm{WT}}$ & $0.96 \pm 0.22$ \\
$\mathrm{AQP} 1_{\mathrm{WT}}+\mathrm{MPK} 2_{\mathrm{K} 42 \mathrm{~A}}$ & $1.14 \pm 0.19$ \\
$\mathrm{AQP} 1_{\mathrm{K} 12 \mathrm{~A}}+\mathrm{MPK} 2_{\mathrm{WT}}$ & $0.97 \pm 0.08$ \\
$\mathrm{AQP} 1_{\mathrm{K} 12 \mathrm{R}}+\mathrm{MPK} 2_{\mathrm{WT}}$ & $1.05 \pm 0.16$ \\
$\mathrm{AQP} 1_{\mathrm{K} 12 \mathrm{~A}}+\mathrm{MPK} 2_{\mathrm{K} 42 \mathrm{~A}}$ & $1.17 \pm 0.18$ \\
$\mathrm{AQP} 1_{\mathrm{K} 12 \mathrm{R}}+\mathrm{MPK}_{\mathrm{K} 42 \mathrm{~A}}$ & $1.03 \pm 0.09$ \\
\hline
\end{tabular}

Thus, it is reasonable to speculate that a lysine at this position of the N-terminus along with phosphorylation at T197 may be required to localize AQP1 to the flagellar membrane. No specific signaling mechanisms for flagellar proteins have been established in kinetoplastids. In this group of parasitic protozoans, a consensus sequence, HLA, is contained within two cytoskeletal flagellar proteins, paraflagellar rod A and trypanosome actin-related protein (Ersfeld and Gull 2001). This sequence, however, is not universal. For example the Leishmania enriettii glucose transporter ISO1 trafficks to the flagellar membrane, whereas ISO2 is routed to the pellicular membrane (Nasser and Landfear 2004). However, AQP1 does not have any such sequences. These are intriguing questions which will be addressed in a separate study very soon by combining our K12 and R13K mutants with T197 mutants of AQP1 and wild type/ MPK2K42A respectively. 
Based on the data obtained so far we propose a model to describe the mechanisms of AQP1 regulation at the post-translational level by probable ubiquitination and MPK2 intervention (Figure 29).

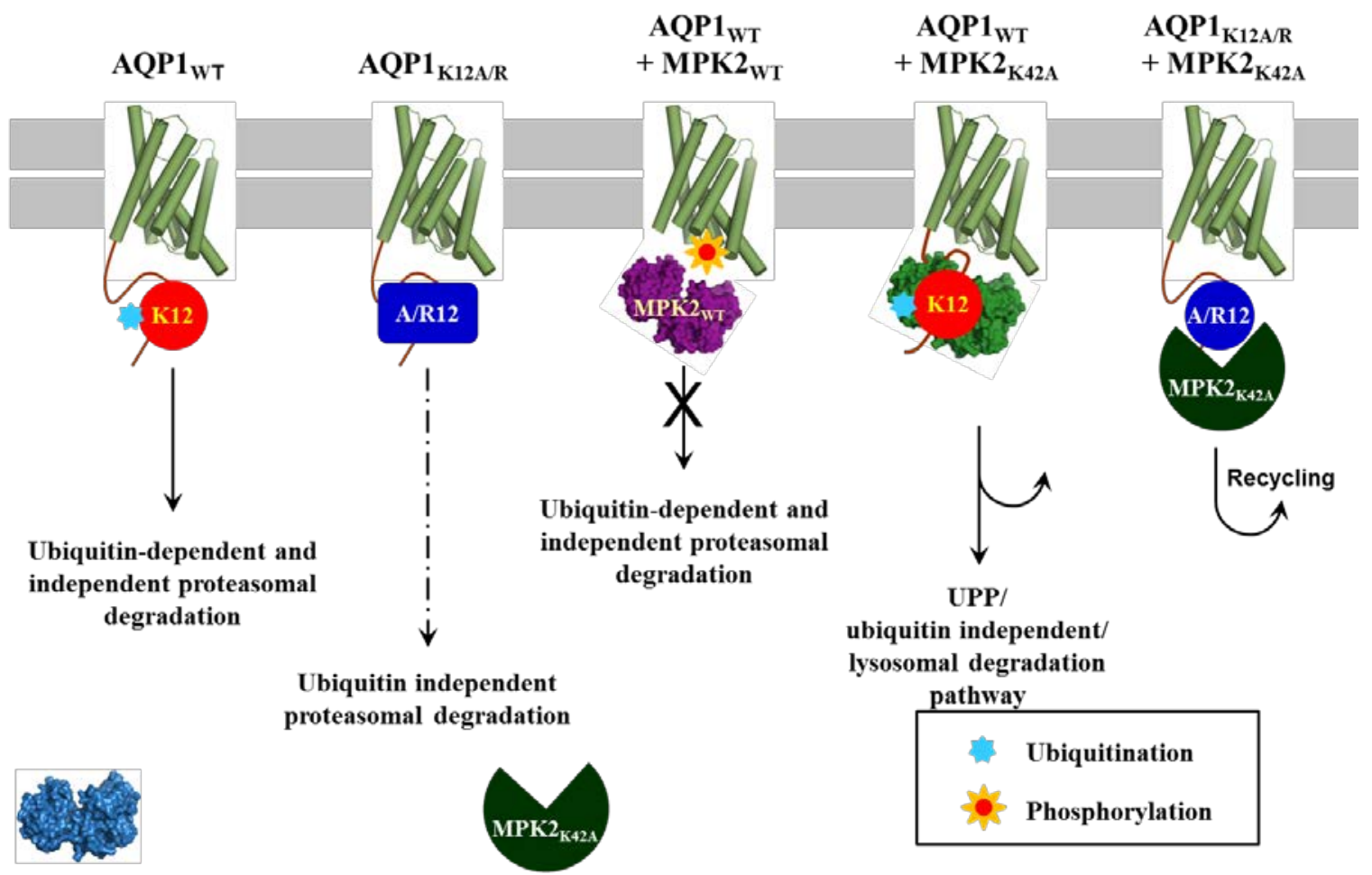

Figure 29. Proposed mechanism of AQP1 regulation at the post-translational level by probable ubiquitination and MPK2 intervention. 
When wild type AQP1 is present in the flagellar membrane in AQP1 alone cells, a steady state exists among synthesis, flagellar trafficking, recycling and degradation through proteasomal and/or endosomal-lysosomal pathways. In AQP1к12A/AQP1к12R alone cells, this steady state may still be functional, as we observed somewhat increased stability of altered AQP1 more so than in AQP1 $1_{12 R}$ (Figure 26), most likely because AQP1 cannot get ubiquitinated at this point. However, these cells may maintain the steady state by degrading AQP1 through ubiquitin-independent pathways, such as described for intrinsically disordered proteins (Erales and Coffino 2014; Sanchez-Lanzas and Castano 2014); as the N-terminus is still flip-floppy. In AQP1wT+MPK2wT cells, MPK2 phosphorylates AQP1. As a result of this close association and structural changes in AQP1, the N-terminus assumes an ordered structure and also masks K12, leading to stabilization. The recycling continues between the "on" (during phosphoryl transfer to AQP1) and "off" (release of AQP1 i.e. the substrate after phosphorylation is complete) phases of MPK2 association with AQP1.

Now, let us consider the case of accelerated degradation of AQP1 in the presence of MPK2 ${ }_{\mathrm{K} 42 \mathrm{~A}}$ (Figure 26). It may be possible that the altered MPK2 docks onto AQP1 and locks into an "on" position without the phosphorylation mediated transformation and release of AQP1. This may expose K12 for ubiquitination again and may result in a hyper-flipping N-terminus, which leads to accelerated degradation through both proteasomal dependent and independent pathways. The recycling and trafficking events are blocked because of constant "on" association of MPK2 $442 \mathrm{~A}$ with AQP1, and hence we observe a complete destabilization. However, when K12 is removed from this equation, 
ubiquitination mediated degradation does not take place. Recycling and trafficking may also resume as MPK2 $442 \mathrm{~A}$ may be increasing order into that $\mathrm{N}$-terminus in the absence of any ubiquitination. It is also possible that other interacting partners of AQP1 are involved such as E3 ubiquitin ligases. Experiments in support of this hypothesis and identification of additional partners of AQP1 should be completed in future.

In the absence of any transcriptional control in Leishmania, such posttranslational regulation(s) of AQP1 provides the parasite a highly competent way to combat the osmotic challenges it faces during its life cycle and transmission. It is tempting to speculate that upregulation of AQP1 by wild type MPK2 is a plus during a hypo-osmotic challenge right after transmission. However, soon after, especially during a drug exposure, the parasite may use the intrinsically disordered $\mathrm{N}$-terminus with the critical K12 at a strategic position to remove it from the membrane and downregulate AQP1 to become drug resistant. 


\subsection{References}

Bastin, P., Pullen, T. J., Moreira-Leite, F. F., \& Gull, K. (2000). Inside and outside of the trypanosome flagellum: a multifunctional organelle. Microbes and Infection, 2(15), 1865-1874.

Decuypere, S., Rijal, S., Yardley, V., De Doncker, S., Laurent, T., Khanal, B., et al. (2005). Gene expression analysis of the mechanism of natural Sb(V) resistance in Leishmania donovani isolates from Nepal. Antimicrobial Agents and Chemotherapy, 49(11), 4616-4621.

Erales, J., \& Coffino, P. (2014). Ubiquitin-independent proteasomal degradation. Biochimica et biophysica acta, 1843(1), 216-221.

Ersfeld, K., \& Gull, K. (2001). Targeting of cytoskeletal proteins to the flagellum of Trypanosoma brucei. Journal of Cell Science, 114 (1), 141-148.

Figarella, K., Uzcategui, N. L., Zhou, Y., Lefurgey, A., Ouellette, M., Bhattacharjee, H., et al. (2007). Biochemical characterization of Leishmania major aquaglyceroporin LmAQP1: possible role in volume regulation and osmotaxis. Molecular Microbiology, 65(4), 1006-1017.

Frezard, F., Demicheli, C., \& Ribeiro, R. R. (2009). Pentavalent antimonials: new perspectives for old drugs. Molecules, 14(7), 2317-2336.

Gourbal, B., Sonuc, N., Bhattacharjee, H., Legare, D., Sundar, S., Ouellette, M., et al. (2004). Drug uptake and modulation of drug resistance in Leishmania by an aquaglyceroporin. The Journal of Biological Chemistry, 279(30), 31010-31017.

Goyard, S., Segawa, H., Gordon, J., Showalter, M., Duncan, R., Turco, S. J., et al. (2003). An in vitro system for developmental and genetic studies of Leishmania donovani phosphoglycans. Molecular and Biochemical Parasitology, 130(1), 31-42.

Gull, K. (1999). The cytoskeleton of trypanosomatid parasites. Annual Review of Microbiology, 53, 629-655.

Hill, K. L. (2003). Biology and mechanism of trypanosome cell motility. Eukaryotic Cell, 2(2), 200-208.

Ishida, T., \& Kinoshita, K. (2007). PrDOS: prediction of disordered protein regions from amino acid sequence. Nucleic Acids Res, 35(Web Server issue), W460-464.

Kamsteeg, E. J., Hendriks, G., Boone, M., Konings, I. B., Oorschot, V., van der Sluijs, P., et al. (2006). Short-chain ubiquitination mediates the regulated endocytosis of the 
aquaporin-2 water channel. Proceedings of the National Academy of Sciences, 103(48), 18344-18349.

Kazemi-Rad, E., Mohebali, M., Khadem-Erfan, M. B., Saffari, M., Raoofian, R., Hajjaran, H., et al. (2013). Identification of antimony resistance markers in Leishmania tropica field isolates through a cDNA-AFLP approach. Experimental Parasitology, 135(2), 344-349.

Landre, V., Pion, E., Narayan, V., Xirodimas, D. P., \& Ball, K. L.(2013). DNA-binding regulates site-specific ubiquitination of IRF-1. Biochemical Journal, 449(3), 707717.

Leitch, V., Agre, P., \& King, L. S. (2001). Altered ubiquitination and stability of aquaporin-1 in hypertonic stress. Proceedings of the National Academy of Sciences, 98(5), 2894-2898.

Li, X., Huang, T., Jiang, G., Gong, W., Qian, H., \& Zou, C. (2013). Proteasome inhibitor MG132 enhances TRAIL-induced apoptosis and inhibits invasion of human osteosarcoma OS732 cells. Biochemical and Biophysical Research Communications, 439(2), 179-186.

Livak, K. J., \& Schmittgen, T. D. (2001). Analysis of relative gene expression data using real-time quantitative PCR and the 2(-Delta Delta C(T)) Method. Methods, 25(4), $402-408$.

Mandal, G., Mandal, S., Sharma, M., Charret, K. S., Papadopoulou, B., Bhattacharjee, H., et al. (2015). Species-specific antimonial sensitivity in Leishmania is driven by post-transcriptional regulation of AQP1. PLoS Neglected Tropical Diseases, 9(2), e0003500.

Mandal, G., Sharma, M., Kruse, M., Sander-Juelch, C., Munro, L. A., Wang, Y., et al. (2012). Modulation of Leishmania major aquaglyceroporin activity by a mitogenactivated protein kinase. Molecular Microbiology, 85(6), 1204-1218.

Mandal, S., Maharjan, M., Singh, S., Chatterjee, M., \& Madhubala, R. (2010). Assessing aquaglyceroporin gene status and expression profile in antimony-susceptible and resistant clinical isolates of Leishmania donovani from India. Journal of Antimicrobial Chemotherapy, 65(3), 496-507.

Marquis, N., Gourbal, B., Rosen, B. P., Mukhopadhyay, R., \& Ouellette, M. (2005). Modulation in aquaglyceroporin $A Q P 1$ gene transcript levels in drug-resistant Leishmania. Molecular Microbiology, 57(6), 1690-1699. 
Mishra, J., Madhubala, R., \& Singh, S. (2012). Visceral and post-Kala-Azar dermal leishmaniasis isolates show significant difference in their in vitro drug susceptibility pattern. Parasitology Research, 112(3), 1001-1009.

Moeller, H. B., Olesen, E. T., \& Fenton, R. A. (2011). Regulation of the water channel aquaporin-2 by post-translational modification. The Americal Journal of Physiology- Renal Physiology, 300(5), F1062-1073.

Mukhopadhyay, R., Mandal, G., Atluri, V. S., Figarella, K., Uzcategui, N. L., Zhou, Y., et al. (2011). The role of alanine 163 in solute permeability of Leishmania major aquaglyceroporin LmAQP1. Molecular and Biochemical Parasitology, 175(1), 83-90.

Nasser, M. I., \& Landfear, S. M. (2004). Sequences required for the flagellar targeting of an integral membrane protein. Molecular and Biochemical Parasitology, 135(1), 89-100.

Papadopoulou, B., Roy, G., \& Ouellette, M. (1992). A novel antifolate resistance gene on the amplified H circle of Leishmania. EMBO Journal, 11(10), 3601-3608.

Perez-Pertejo, Y., Alvarez-Velilla, R., Estrada, C. G., Balana-Fouce, R., \& Reguera, R. M. (2011). Leishmania donovani: proteasome-mediated down-regulation of methionine adenosyltransferase. Parasitology, 138(9), 1082-1092.

Plourdea M, U. J., Mandal G, Monte-Netoa R, Mukhopadhyay R and Ouellette M (2015). Generation of an aquaglyceroporin AQP1 null mutant in Leishmania major. Molecular and Biochemical Parasitology, 201(2), 108-111.

Radivojac, P., Vacic, V., Haynes, C., Cocklin, R. R., Mohan, A., Heyen, J. W., et al. (2010). Identification, analysis, and prediction of protein ubiquitination sites. Proteins, 78(2), 365-380.

Rai, S., Bhaskar, Goel, S. K., Nath Dwivedi, U., Sundar, S., \& Goyal, N. (2013). Role of efflux pumps and intracellular thiols in natural antimony resistant isolates of Leishmania donovani. PLoS ONE, 8(9), e74862.

Sanchez-Lanzas, R., \& Castano, J. G. (2014). Proteins directly interacting with mammalian 20S proteasomal subunits and ubiquitin-independent proteasomal degradation. Biomolecules, 4(4), 1140-1154.

Schaffner, W., \& Weissmann, C. (1973). A rapid, sensitive, and specific method for the determination of protein in dilute solution. Analytical Biochemistry, 56(2), 502514. 
Sundar, S., More, D. K., Singh, M. K., Singh, V. P., Sharma, S., Makharia, A., et al. (2000). Failure of pentavalent antimony in visceral leishmaniasis in India: report from the center of the Indian epidemic. Clinical Infectious Diseases, 31(4), 11041107.

Ukekawa, R., Maegawa, N., Mizutani, E., Fujii, M., \& Ayusawa, D. (2004). Proteasome inhibitors induce changes in chromatin structure characteristic of senescent human fibroblasts. Bioscience, Biotechnology and Biochemistry, 68(11), 23952397.

Umenishi, F., Narikiyo, T., \& Schrier, R. W. (2005). Effect on stability, degradation, expression, and targeting of aquaporin-2 water channel by hyperosmolality in renal epithelial cells. Biochemical and Biophysical Research Communication, 338(3), 1593-1599.

Uzcategui, N. L., Zhou, Y., Figarella, K., Ye, J., Mukhopadhyay, R., \& Bhattacharjee, H. (2008). Alteration in glycerol and metalloid permeability by a single mutation in the extracellular C-loop of Leishmania major aquaglyceroporin LmAQP1. Molecular Microbiology, 70(6), 1477-1486.

Vaughan, S., \& Gull, K. (2003). The trypanosome flagellum. Journal of Cell Science, 116(Pt 5), 757-759.

Vittal, V., Shi, L., Wenzel, D. M., Scaglione, K. M., Duncan, E. D., Basrur, V., et al. (2015). Intrinsic disorder drives N-terminal ubiquitination by Ube2w. Nature Chemical Biology, 11(1), 83-89.

Willermain, F., Janssens, S., Arsenijevic, T., Piens, I., Bolaky, N., Caspers, L., et al. (2014). Osmotic stress decreases aquaporin-4 expression in the human retinal pigment epithelial cell line, ARPE-19. International Journal of Molecular Medicine, 34(2), 533-538.

Yang, Z. R., Thomson, R., McNeil, P., \& Esnouf, R. M. (2005). RONN: the bio-basis function neural network technique applied to the detection of natively disordered regions in proteins. Bioinformatics, 21(16), 3369-3376. 


\title{
CHAPTER 3
}

\section{ROLE OF 3'-UTR IN POST-TRANSCRIPTIONAL REGULATION OF AQP1 IN DIFFERENT SPECIES OF LEISHMANIA}

\author{
This study has been published: \\ Mandal, G., Mandal, S., Sharma, M., Charret, K.S., Papadopoulou, B., \\ Bhattacharjee, H., and Mukhopadhyay, R. Species-Specific Antimonial Sensitivity in \\ Leishmania is driven by Post-Transcriptional Regulation of AQP1 (2015). PLoS \\ Neglected Tropical Diseases. Vol 10.1371/ 0003500
}




\subsection{Introduction}

Leishmaniasis is caused by a digenetic parasite, which belongs to the genus Leishmania. The disease affects almost 12 million people worldwide (Uzcategui et al., 2008). Female sandflies of the genus Phlebotomus and Lutzomyia are responsible for the transmission of the disease in the Old World and New World, respectively. Life cycle of Leishmania include two morphologically distinct forms, namely, promastigote and amastigote. Promastigotes are slender, spindle-shaped bodies with long anterior flagella that reside inside the gut of sandflies. Once inside the vertebrate macrophages, promastigotes are transformed into amastigotes. Amastigotes are small, oval-shaped, aflagelleted structures that reside inside the phagolysosomes of macrophages, proliferate by binary fission and finally burst out of infected cells and infect fresh macrophages, thus perpetuating the disease (Figarella et al., 2007). Leishmaniasis ranges from self-healing cutaneous leishmaniasis (CL) to more debilitating and disfiguring mucocutaneous leishmaniasis (MCL) to fatal visceral leishmaniasis (VL). Cutaneous leishmaniasis affects the skin by causing sores that usually occur at the site of the bite of the sandfly. Visceral leishmaniasis affects the visceral organs, primarily spleen, liver and bone marrow. Mucocutaneous leishmaniasis affects the nasal and oral mucosal membrane. Localized CL caused by L. tropica and L. major usually affects areas of the face, neck and arms. In diffuse Cutaneous leishmaniasis, which is caused by L. aethiopica and $L$ .amazonensis (Mandal et al., 2014), the lesions formed on the body spread from one site to another. The MCL infection, which is due to L. braziliensis, starts as Cutaneous leishmaniasis but some individual parasites spread into the mucous layer of the nasopharynx and cause mutilation of soft cartilage, leading to problems in the nose, 
mouth and throat cavities (Mandal et al., 2014); MCL is fatal unless treated actively. VL, also fatal if left untreated, is caused by L. donovani, L. infantum and L.chagasi. The symptoms of VL include fever, hepatosplenomegaly, lymphoadenopathy and hyperglobulinemia. At later stages, when hyperpigmentation of skin occurs, the levels of IgM and IgG increase and cell-mediated immunity decreases, which increases opportunities for multiple secondary infections. People suffering from HIV are more susceptible to VL (Mandal et al., 2010). Pentavalent antimonite- Sodium stibogluconate (Pentostam) and Meglumine antimonite (Glucantime) are the first lines of treatment against all forms of leishmaniasis (Croft and Coombs 2003). However, drug resistance remains a major barrier to effective treatment of all forms of leishmaniasis.

Pentavalent antimony $\mathrm{Sb}(\mathrm{V})$ is the prodrug that gets reduced to $\mathrm{Sb}(\mathrm{III})$, which is the active form of the drug. Reduction of $\mathrm{Sb}(\mathrm{V})$ metal may take place either in the parasite or in the macrophages or both. Host macrophages play important roles in the reduction (Frezard et al., 2009). Arsenic and antimony have similar properties and are transported via similar channels and pumps. It was reported that one part of $\mathrm{Sb}(\mathrm{V})$ is reduced to $\mathrm{Sb}(\mathrm{III})$ by leishmanial $\mathrm{As}(\mathrm{V})$ reductase (Figure 30) or LmACR2 (Zhou et al., 2006). The resultant $\mathrm{Sb}(\mathrm{III})$ is conjugated to trypanothione (TSH) and is either exported out by an unknown efflux pump (Mukhopadhyay et al., 1996) and/or sequestered into small intracellular vesicles near the flagellar pocket of MRPA (Legare et al., 1997). These mechanisms seem to play substantial roles in Leishmania and also in field isolates. Haldar et al., 2011 reported that variability in the frequency of clinical antimonial resistance exists amongst Leishmania species. However, it was not known yet whether 
an intrinsic variation in antimonial sensitivity occurs among different Leishmania species. Gourbal et al., 2004 reported that $L$. major was 50-70 times more sensitive to antimonite when compared to L. infantum. It was also reported that Leishmania strains causing self-healing Cutaneous leishmaniasis have greater susceptibility towards oxidative stress as they have reduced thiol content (Sarkar et al., 2012). However, a species-wide study has not been conducted, and the cause of this resistance remains unknown.

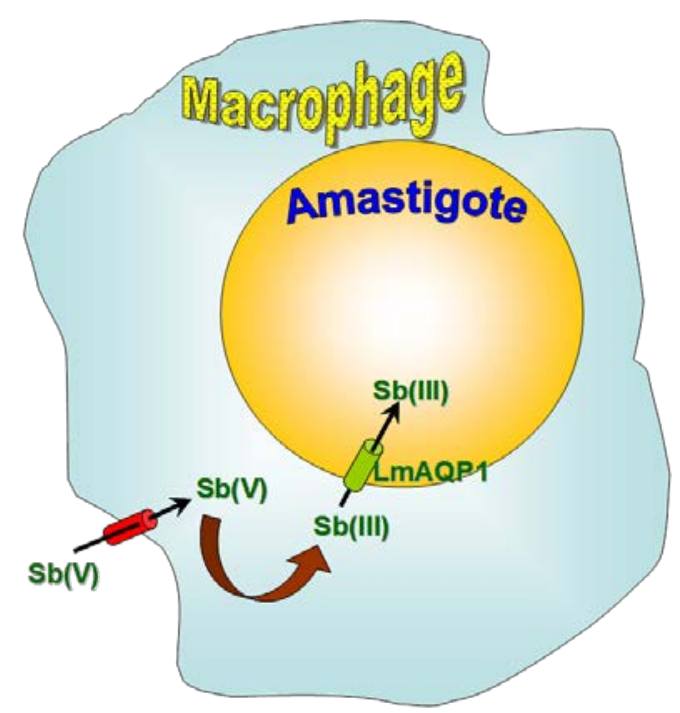

Figure 30. Model of Pentostam action in macrophage associated amastigotes of Leishmania. $\mathrm{Sb}(\mathrm{V})$ is taken up by macrophages, and a portion is reduced to $\mathrm{Sb}$ (III), which is then transported into the amastigote by AQP1.

To date, Leishmania aquaglyceroporin AQP1 is the only reported Sb(III) facilitator (Gourbal et al., 2004). Disruption of one of the two AQP1 alleles in L. major resulted in a ten-fold increase in resistance to Sb(III) (Marquis et al., 2005). This has been corroborated by downregulation of AQP1 mRNA levels in clinical isolates of drug resistant $L$. donovani. On the other hand, overexpression of AQP1 makes the wild-type 
parasites 100-fold more sensitive to Sb(III). More importantly, overexpression of AQP1 can abolish the drug resistance phenotype in drug resistant isolates (Gourbal et al., 2004) Taken together, AQP1 plays a significant role in the successful treatment of leishmaniasis using antimonials. Other factors, such as levels of trypanothione and antimony-TSH conjugate exporter (Mukhopadhyay et al., 1996), also play important roles in the generation of drug resistance.

During our study with AQP1, we noticed that visceral species of Leishmania are much more antimonite (SbIII) resistant as compared to mucocutaneous and cutaneous species. As we stated previously, AQP1 is the lone facilitator of $\mathrm{Sb}(\mathrm{III})$ in Leishmania, so we were curious to learn whether species-specific antimony resistance is driven by AQP1. Trypanosomes, including Leishmania, do not have promoters, unlike other eukaryotes for RNA polymerase II, which is involved in the transcription of proteincoding genes and is sensitive to $\alpha$-amanitin. Instead, these protein-coding genes are constitutively transcribed from large gene clusters, and steady state levels of their mature mRNAs are regulated post-transcriptionally by trans-splicing and polyadenylation (Kramer 2012 ; Haile and Papadopoulou 2007). Several studies reported that posttranscriptional regulation of developmentally expressed transcripts in Leishmania species involves sequences present mainly in the 3'-UTR (Muller et al., 2010 ; Boucher et al., 2002; McNicoll et al., 2005; Bringaud et al., 2007), but also in the 5’UTR (Aly et al., 1994; Lamontagne and Papadopoulou 1999) and in intergenic regions between tandemly repeated genes (Ramamoorthy et al., 1995; Beetham et al., 1997). Logarithmic-stationary gene regulation is also regulated by 3'-UTR (Myung et al., 2002). 
In this study, the 3'-UTRs of AQP1 were mapped and cloned from six different Leishmania species. Each 3'-UTR fragment was approximately $1.8 \mathrm{~kb}$ in length, highly U-rich (30\%) with only 49\% GC (in a highly GC-rich genome), and contained several well-known instability elements described in higher eukaryotes. Each3'-UTR fragment was very complex with several unique features: AU-rich (ARE) regions (highlighted in yellow), CU-rich (CURE) regions (highlighted in cyan), and Short Tandem Repeats (STRs, highlighted in green, Figure 31).

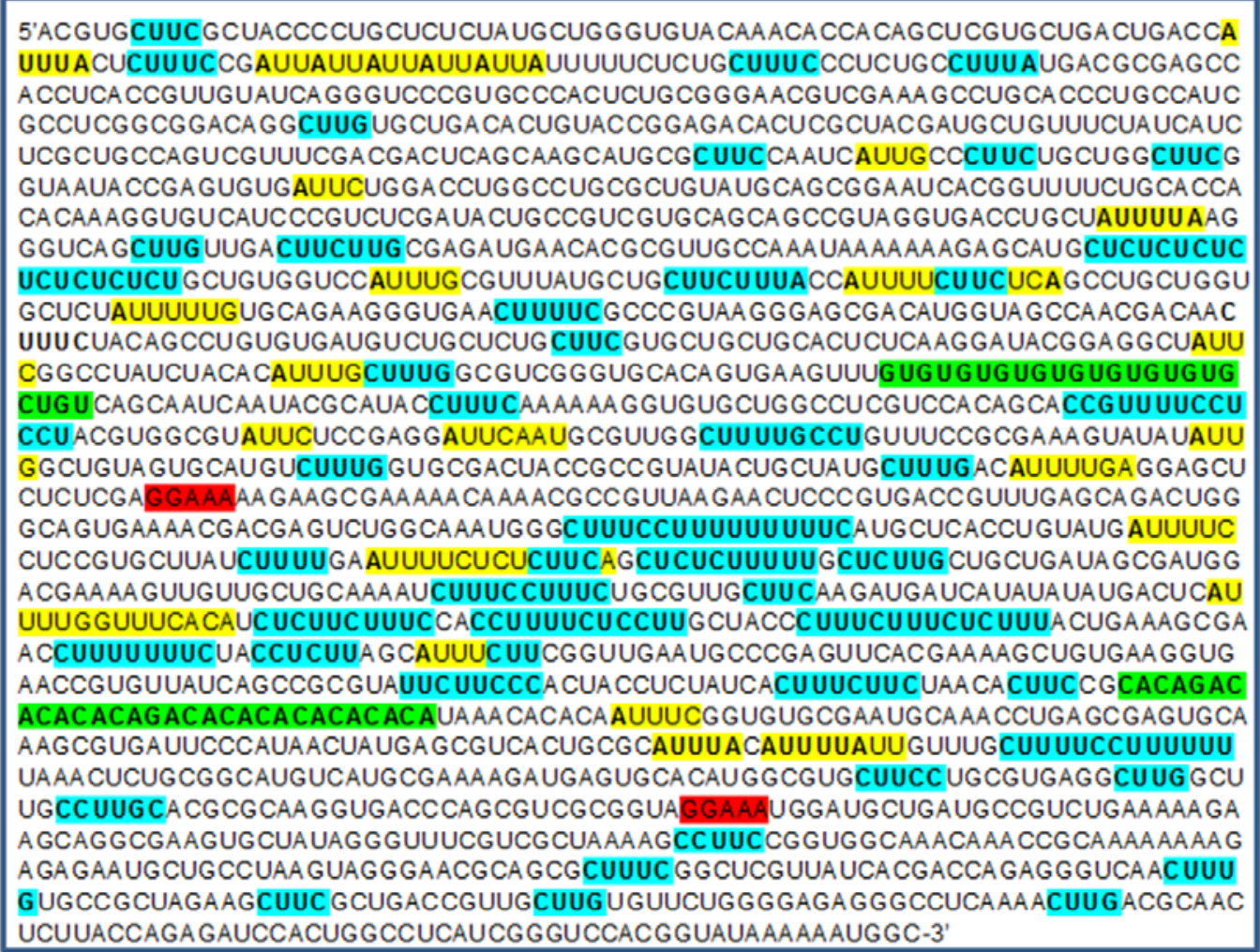

Figure 31. 3'UTR sequence of AQP1 from L. donovani. Yellow : ARE, Cyan : CURE, Green : STR., Red: NFAT motif

Sometimes ARE and CURE sequences overlap. ARE regions are expected to destabilize mRNA (Winzen et al., 2007, Otkjaer et al., 2010), and U-rich UTRs make 
transcripts unstable (Rodrigues et al., 2010). It is interesting to note that the Lm3'-UTR has more AU-rich regions than the Ld3'-UTR, although the stretches of CU-rich regions are essentially the same. Although the protein sequences of AQP1 among these six species are more than $80 \%$ homologous, their 3'-UTR differs significantly. The 3'UTRs of AQP1 were mapped and cloned from six species: L. donovani (Ld3'-UTR), L. major (Lm3'-UTR), L. infantum (Li3'-UTR), L. tropica (Lt3'-UTR), L. braziliensis (Lb3'-UTR) and L. panamensis (Lp3'-UTR). The rationale behind choosing different species was to represent every endemic continent (Asia [L. donovani, L. infantum, L. major and L. tropica], Africa [same as Asia], Europe [L. infantum, L. major and L. tropica] and America [L. infantum, L. braziliensis and L. panamensis)]); clinical manifestation (VL- L. donovani, L. infantum; Cutaneous leishmaniasis- rest of the species; MCL- L. braziliensis); and mode of transmission (anthroponotic- L. donovani and L. tropica; zoonotic- rest of the species) (Mandal et al., 2015). This study shows that the species-specific antimonial resistance in Leishmania is uniquely driven by AQP1 and the post-transcriptional regulation is modulated by the respective unique 3'-UTR of each species-specific AQP1.

\subsection{Materials and methods}

\subsubsection{Cell culture and infections}

Leishmania donovani strain LdBob, L. infantum, L. major strain LV39 and L. braziliensis were kind gifts from Professor Stephen M. Beverley from Washington University School of Medicine; Professor Barbara Papadopoulou and Professor Marc Ouellette from Laval University, Quebec; and Professor Kwang-Poo Chang from 
Rosalind Franklin University . L. tropica and L. panamensis were purchased from ATCC.

Leishmania promastigotes were grown and maintained as described earlier in section

2.2.1 (Figarella et al., 2007,Uzcategui et al., 2008) as well as maintained on blood agar slant brain heart infusion broth biphasic medium. Blood agar slants were prepared using 3.7\% brain heart infusion (BHI), 1.7\% agar and defibrinated rabbit blood.

\subsubsection{Antimony sensitivity assay}

Antimony sensitivity assay of the promastigote was described previously in Section 2.2.4 (Chapter 2) (Sharma et al., 2015)

\subsubsection{Uptake assay}

The transport assay was performed using log phase $\left(10^{8} \mathrm{cells} / \mathrm{ml}\right)$ promastigotes. Cells were washed twice with PBS, pH 7.4 (Thermo scientific) and resuspended in PBS. Promastigote cells were incubated with $10 \mathrm{mM}$ potassium antimonyl tartarate [Sb(III)] for different time points at room temperature. At each time point, 200 $\mu$ l of cells were filtered through a nitrocellulose membrane, and the filters were washed with $5 \mathrm{ml}$ of icecold PBS. The intracellular contents of antimony were determined as described previously (Gourbal et al., 2004).

Membrane vesicles were prepared from promastigotes of each species as described previously (Mukhopadhyay et al., 1996). They were rapidly frozen in liquid nitrogen in small aliquots and stored at $-80^{\circ} \mathrm{C}$ until use. The total protein content of the plasma membrane fractions was determined by a filter assay as described previously (Schaffner and Weissmann 1973). ATP-dependent uptake of Sb(TS)2 was measured 
with $10 \mathrm{mM}$ ATP as the energy source, as described previously, with a few changes (Mukhopadhyay et al., 1996). Briefly, vesicles were added at $0.5 \mathrm{mg}$ of membrane protein/ml and incubated with $0.1 \mathrm{mM}$ of $\mathrm{Sb}(\mathrm{TS})_{2}$ in a buffer containing 75MM HEPES$\mathrm{KOH}, \mathrm{pH}$ 7.0/0.15M KCl. The reaction was started by the addition of ATP at room temperature. At the indicated intervals, samples $(0.1 \mathrm{ml})$ were removed and filtered on a wet $0.22 \mu \mathrm{m}$ nitrocellulose filter, and the filter was washed once with $5 \mathrm{ml}$ of ice-cold PBS. The membranes were digested with $70 \% \mathrm{HNO}_{3}$, and total $\mathrm{Sb}$ content was measured by ICP-MS as described above. Each transport experiment was repeated at least two times with triplicate samples. Error bars were calculated from the mean $\pm \mathrm{SE}$.

\subsubsection{Cell volume measurements}

Relative changes in cell volume were measured upon introduction of hypoosmotic stress. Please refer to section 2.2.6 (Chapter 2) for details (Sharma et al., 2015)

\subsubsection{Isolation of nucleic acids}

DNAzol $^{\mathrm{TM}}$ reagent was used for isolation of genomic DNA. Total RNA from Leishmania promastigote cells was isolated using TRIZOL reagent, according to the manufacturer's protocol. DNA was removed from total RNA preparation using TURBO DNA-free ${ }^{\mathrm{TM}}$ Kit (Ambion), according to the manufacturer's instructions (Müller et al., 2010). The integrity of total RNA preparations was confirmed by denaturing agarose gel electrophoresis. 


\subsubsection{Mapping of 3'-UTR}

The 3'-UTR fragment for each of the six Leishmania species was mapped using 3'RACE kit (Invitrogen) with $2 \mu \mathrm{g}$ of total RNA input and gene-specific primers, according to the manufacturer's protocol. The gene-specific primers were designed according to genome sequences in http:/tritrypdb.org/tritrypdb. The amplified 3'-UTR fragments for all the species were cloned into pGEMT-Easy vector (Promega), according to the manufacturer's instructions, and sequenced (Eton Biosciences) (Mandal et al., 2015).

\subsubsection{Plasmid construction and transfection}

The luciferase (LUC) expression vector for Leishmania pSPYNEOaLUC was described previously (Boucher et al., 2002) and is referred to as $L U C$-control in this study. The full-length 3'-UTR of AQP1 and 200 bp beyond the 3' end of the poly (A) site was PCR amplified from the genomic DNA of each species using AccuPrime ${ }^{\mathrm{TM}}$ Taq DNA Polymerase (Invitrogen) and primers with inserted BamHI or SalI restriction sites. The PCR products were cloned into the pGEMT-Easy vector (Promega) and sequenced (Eton Bioscience). The different $L U C$-chimeric constructs were generated by digesting the pGEMT-Easy clones with BamHI or SalI (New England Biolab) and subcloned into the BamHI or SalI site 3' to the $L U C$ gene in the vector pSPYNEO $\alpha \mathrm{LUC}$, respectively. All plasmid constructs were purified using QIAprep Spin Miniprep Kit (Qiagen). Purified plasmid constructs were transfected into Leishmania by electroporation. Briefly, stationary phase promastigotes were washed and resuspended in an ice-cold electroporation buffer (21 mM HEPES, $150 \mu \mathrm{M} \mathrm{NaCl,} 5$ mM MgCl 2,120 mM KCl, 0.7 
$\mathrm{mM} \mathrm{NaH} \mathrm{PO}_{4}, 6 \mathrm{mM}$ glucose) at a density of $10^{8} \mathrm{cell} / \mathrm{s} / \mathrm{ml}$. Three hundred microliters of resuspended cells were transferred to $0.2 \mathrm{~cm}$ electroporation cuvette (Bio-rad) with $13 \mu \mathrm{g}$ of plasmid DNA. Cells were electroporated in Gene Pulser (Bio-rad) at $0.45 \mathrm{kV}$ and 500 $\mu \mathrm{F}$. All transfectants were selected and maintained in the presence of $60 \mu \mathrm{g} / \mathrm{ml}$ genticin (G418) (Invitrogen).

\subsection{8. qPCR and qRT-PCR analyses}

cDNA synthesis was carried out using 500 ng of total RNA and AccuScript High Fidelity $1^{\text {st }}$ strand cDNA synthesis kit (Agilent), according to the manufacturer's instructions. The first-strand cDNA reaction mix was treated with $0.25 \mathrm{~N} \mathrm{NaOH}$ at $65^{\circ} \mathrm{C}$ for 30 min to degrade the input RNA molecules. The reaction mix was then neutralized using $\mathrm{HCl}$ and purified using Qiagen PCR purification kit, according to the manufacturer's instructions. For qPCR, $4 \mu \mathrm{g}$ of genomic DNA, and for qRT-PCR, $2 \mu \mathrm{l}$ of diluted, purified cDNA reaction corresponding to 6 ng of input RNA, was used in a $10 \mu \mathrm{l}$ reaction containing forward and reverse primers for the target genes and $1 \mathrm{X} \mathrm{iQ}^{\mathrm{TM}} \mathrm{SYBR}$ Green supermix (Bio-rad). The reactions were run on an Eppendorf Realplex ${ }^{2}$ PCR machine in the following thermal cycling conditions: initial denaturation at $95^{\circ} \mathrm{C}$ for 3 min followed by 40 cycles of $95^{\circ} \mathrm{C}$ for 15 sec and $65^{\circ} \mathrm{C}$ for 20 sec. A final melting curve analysis was performed for each reaction to confirm that the PCR generated a single amplification product. The relative abundance of target amplicons between samples were estimated using the $2^{-\Delta \Delta C T}$ method (Mary et al., 2010). Error bars were calculated from the mean \pm SE of three independent experiments in triplicate. 


\subsubsection{RNA stability assays}

To determine the half-lives of AQP1, mid-log phase promastigotes were treated with sinefungin $(5 \mu \mathrm{M})$ for $15 \mathrm{~min}$, followed by incubation with $10 \mu \mathrm{g} / \mathrm{ml}$ of actinomycin D (Sigma) to arrest trans-splicing and transcription. Cells were harvested at various time points. Total RNA isolation and qRT-PCR analyses were performed as described earlier. Error bars were calculated from the mean \pm SE of three independent experiments in triplicate (Boucher et al., 2002).

\subsubsection{Luciferase activity assay}

Mid-log phase promastigotes $\left(5 \times 10^{6}\right)$ were lysed with $50 \mu$ l of lysis buffer $(62.5$ mM Tris-phosphate pH 7.8, 5 mM DTT, 2.5\% Triton X-100, 25\% glycerol). A $10 \mu \mathrm{l}$ of lysate was used to estimate the luciferase activity using Luc-Screen ${ }^{\circledR}$ Extended-Glow Luciferase Reporter Gene Assay System (Life Technologies) according to the manufacturer's instructions. The luciferase activities were normalized against the relative copy number of the plasmid constructs in each transfectant. Error bars were calculated from the mean \pm SE of three independent experiments in triplicate (Mandal et al., 2015).

\subsubsection{Western blot analysis}

Whole cell lysates were prepared by lysing $1 \times 10^{8}$ promastigotes from each transfectant in $100 \mu \mathrm{l} 1 \mathrm{x}$ Laemmli's buffer. A $10 \mu \mathrm{l}$ of lysate was used to fractionate proteins on $12 \%$ SDS-PAGE. Fractionated proteins were electroblotted on nitrocellulose membranes. The amounts of luciferase and neomycin phosphotransferase proteins were 
estimated using goat anti-luciferase pAp (Promega) and rabbit anti-neomycin phosphotransferase antibody, respectively, followed by densitometric estimation (Mandal et al., 2015). .

\section{3. Results}

\subsubsection{Intrinsic species-specific antimonial resistance in Leishmania}

Intrinsic $\mathrm{Sb}(\mathrm{III})$ resistance in the representative six selected species was determined by exposing the promastigotes to increasing concentrations of potassium antimonyl tartrate. L. infantum was the most resistant species. The EC50 data showed that it was 1.4 times more resistant when compared to L. donovani and 46, 15, 20 and 7 times more resistant than the cutaneous species namely, L. major, L. tropica, $L$. braziliensis and L. panamensis, respectively (Table 4). However, the cutaneous species also differed in sensitivity to Sb(III) among themselves. L. tropica, L. braziliensis and $L$. panamensis are 3, 2.3 and 6 times, respectively, more resistant to $\mathrm{Sb}(\mathrm{III})$ when compared to L. major (Table 4).

Table 4. Antimonite sensitivity of different species of Leishmania

\begin{tabular}{|l|c|}
\hline \multicolumn{1}{|c|}{ Species } & $\begin{array}{c}\mathbf{E C}_{\mathbf{5 0}} \\
\mathbf{S b}(\mathbf{I I I}) \boldsymbol{\mu M}\end{array}$ \\
\hline L.braziliensis & $10 \pm 2$ \\
\hline L.donovani & $144 \pm 5$ \\
\hline L.infantum & $199 \pm 22$ \\
\hline L.major & $4.3 \pm 0.3$ \\
\hline L.panamensis & $27 \pm 4$ \\
\hline L.tropica & $13 \pm 1$ \\
\hline
\end{tabular}




\subsubsection{Cutaneous leishmaniasis species accumulate more $\mathrm{Sb}$ (III) than the VL species}

In order to understand differential intrinsic antimonial sensitivity among different species, we examined the time-dependent intracellular accumulation of $\mathrm{Sb}(\mathrm{III})$ in promastigotes. We observed that $L$. braziliensis showed the fastest and highest accumulation of $\mathrm{Sb}(\mathrm{III})$ at any given time followed by L. major, $L$. panamensis and $L$. tropica, whereas $L$. donovani and $L$. infantum showed the slowest rates and total accumulation of Sb(III) (Figure 32).

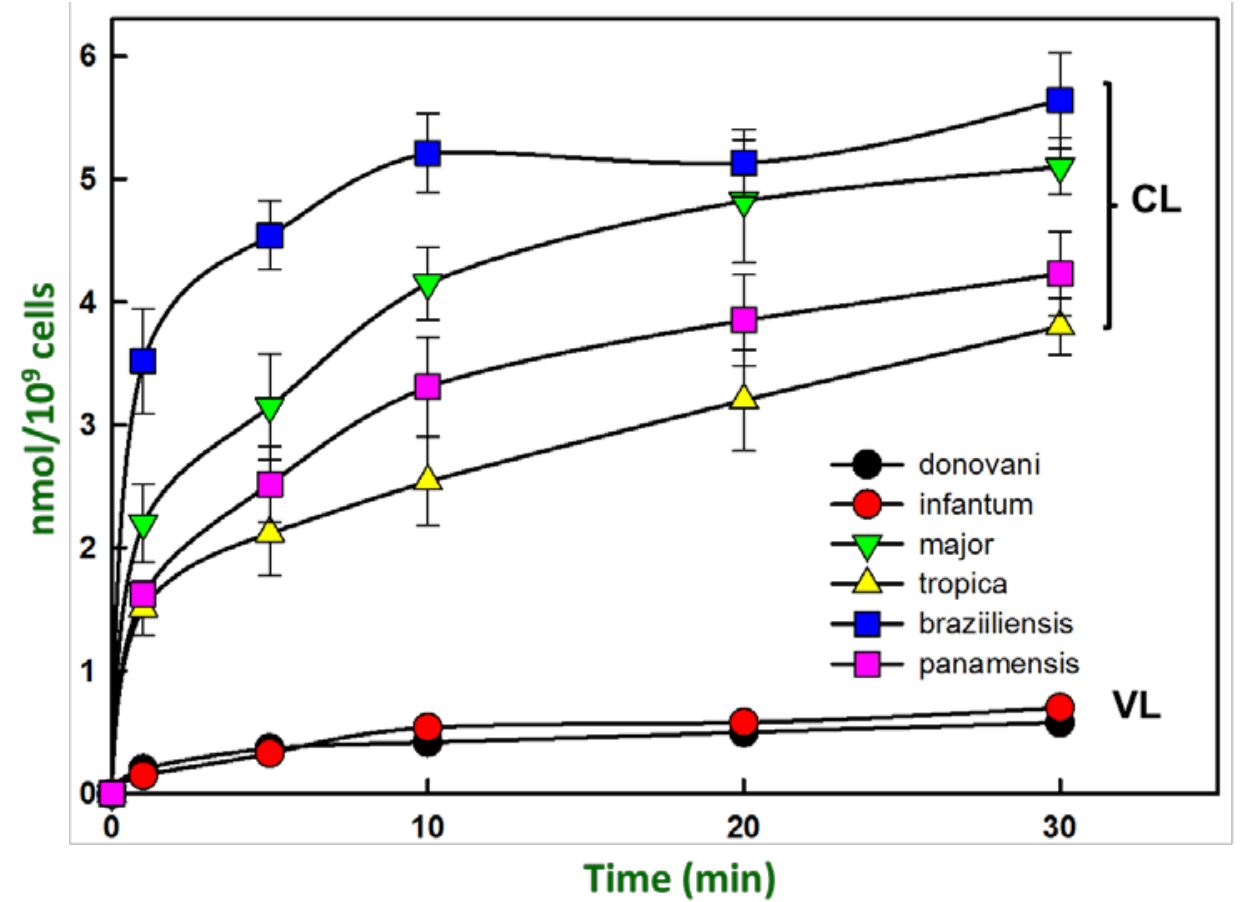

Figure 32. Sb(III) accumulation of promastigotes of different species of Leishmania. Promastigotes of different species of Leishmania were exposed to $0.05 \mathrm{mM}$ potassium antimony tartrate (SbIII). Cells were harvested at different time points and antimony accumulation was estimated using ICP-MS. Data were expressed as mean \pm SEM of three independent experiments in duplicate. 
Therefore, our studies indicated that the antimony sensitive Cutaneous leishmaniasis species accumulated more total antimony when compared to the VL species. Our next question was whether the accumulation signified lower uptake or increased efflux in the VL species and higher uptake or slower efflux in the Cutaneous leishmaniasis species. To address this question, everted membrane vesicles were prepared from promastigotes of each species, and Sb-[TS $]_{2}$ conjugate accumulation was

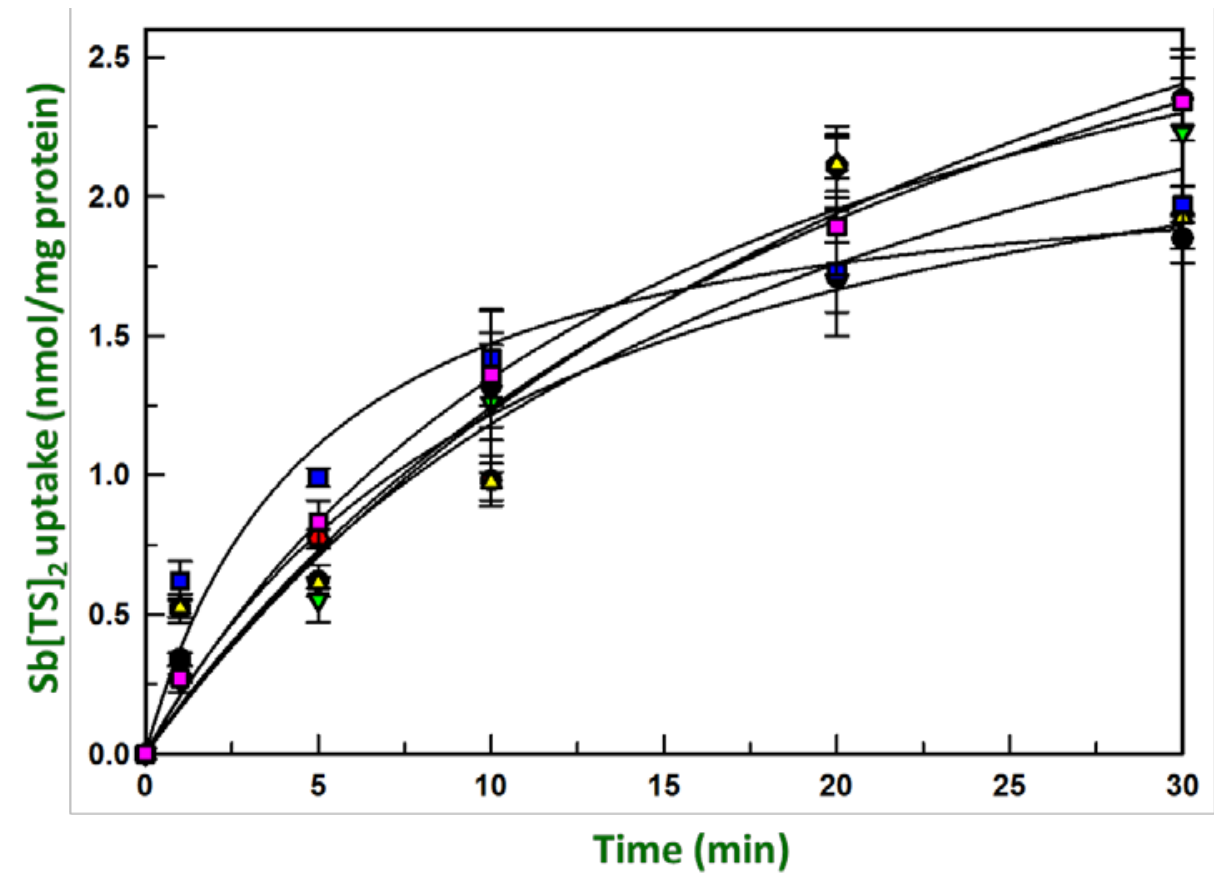

Figure 33. Efflux of Sb-trypanothione complex in plasma membrane vesicles isolated from different species of Leishmania. Everted plasma membrane enriched vesicles were prepared from promastigotes of different species and transport assayed with $0.1 \mathrm{mM} \mathrm{Sb}(\mathrm{TS})_{2}$ and $10 \mathrm{mM}$ ATP as energy source. The values at each time point were corrected for non-specific binding by subtraction of the values obtained with $10 \mathrm{mM}$ AMP. Data were expressed as mean \pm SE of three independent experiments in triplicate.

measured in the presence of $10 \mathrm{mM}$ ATP as an energy source. The rate of transport of Sb$[\mathrm{TS}]_{2}$ conjugate in the everted membrane vesicles of the six different species was not significantly different from each other, which indicated that differential sensitivity among different species was not due to a change in the efflux rate of antimony (Figure 33). 


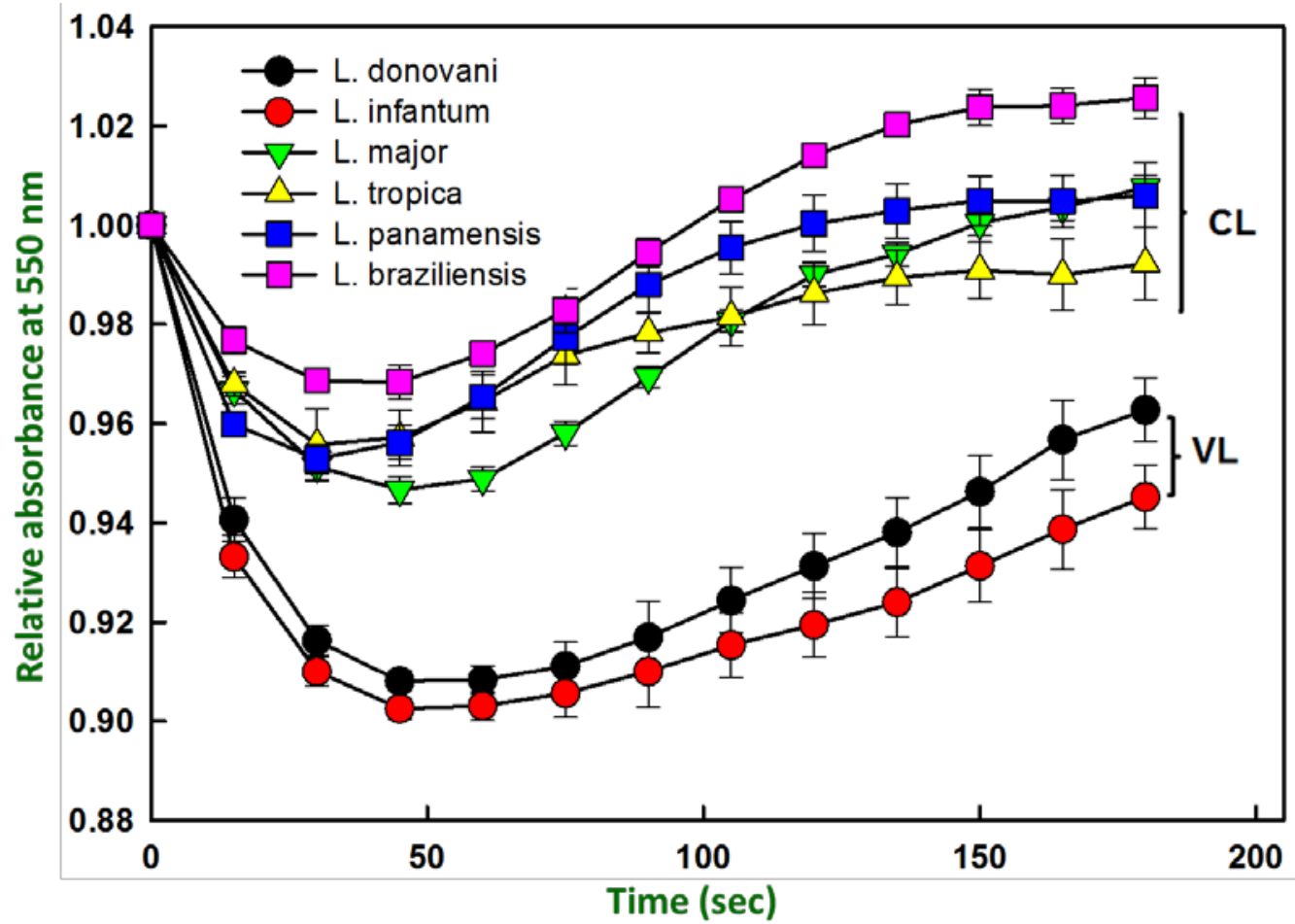

Figure 34. Volume regulation of promastigotes of different species of Leishmania under hypoosmotic shock. Promastigotes of different species of Leishmania were subjected to a hypo-osmotic shock, and the relative changes in cell volume were followed by monitoring the absorbance at $550 \mathrm{~nm}$. Data were expressed as mean \pm SEM of three independent experiments in duplicate.

\subsubsection{Osmoregulatory capacity of promastigotes is species-specific}

Mukhopadhyay's lab has previously shown that the physiological function of AQP1 in Leishmania is osmoregulation. AQP1's strategic localization in the flagella helped the parasite to sense the changing osmotic environment between the vector and the host (Figarella et al., 2007). In wild type Leishmania, it was very difficult to detect AQP1 expression at the protein level with an antipeptide polyclonal anti-AQP1 antibody (Figarella et al., 2007). Therefore, it is important to understand the significance of AQP1 in all six species by determining their osmoregulatory capacities under hypo-osmotic 
shock (50\% reduction in osmolarity). It was previously reported that osmoregulatory capacity is directly proportional to the AQP1 protein levels (Figarella et al., 2007, Mandal et al., 2012) in the membrane. We observed that the Cutaneous leishmaniasis species osmoregulates much more efficiently when compared to the VL species as predicted by their EC50. Studies show that VL species promastigotes with less AQP1 mRNA are more resistant to antimonials when compared to Cutaneous leishmaniasis species promastigotes with more AQP1 mRNA. We observed that $L$. donovani and $L$. infantum promastigotes swelled more rapidly (decrease in absorbance) and recovered their volume (increase in absorbance) more slowly when compared to all the Cutaneous leishmaniasis species (Figure 34). Mandal et al., 2015 reported that $L$. braziliensis and $L$. panamensis showed the highest levels of AQP1 mRNA and that they were the most efficient osmoregulators, whereas $L$. infantum, with less AQP1 mRNA compared to $L$. donovani, responds to hypo-osmotic shock less efficiently.

\subsubsection{AQP1 mRNA is more stable in the Cutaneous leishmaniasis species}

Leishmania does not have any transcriptional control. Thus, we examined the stability of AQP1 mRNA in all six species to determine if any post-transcriptional regulation is active in lowering the mRNA levels in the VL species. In order to determine the turnover rate of AQP1 mRNA, mid log phase promastigotes of all species were treated with sinefungin (to stop pre-mRNA processing) (Mayer et al., 2012), followed by actinomycin D (inhibition of transcription) (Mishra et al., 2003), and then harvested up to 130 min. We used quantitative PCR (qPCR) to determine the decay of AQP1 mRNA and normalized against the 0 minute (time of addition of actinomycin D). 
The half-lives of the AQP1 mRNA in VL species, i.e. $L$ donovani and $L$. infantum, were found to be $40 \mathrm{~min}$ and $26 \mathrm{~min}$, respectively, whereas the half-lives of the Cutaneous leishmaniasis species, such as L. tropica and L. panamensis, were 57 and $117 \mathrm{~min}$, respectively. The half-lives of the AQP1 mRNA in L. braziliensis and L. major were estimated to be $>130 \mathrm{~min}$ (Figure 35). Therefore, AQP1 mRNA in the Cutaneous leishmaniasis species was much more stable than the VL species.

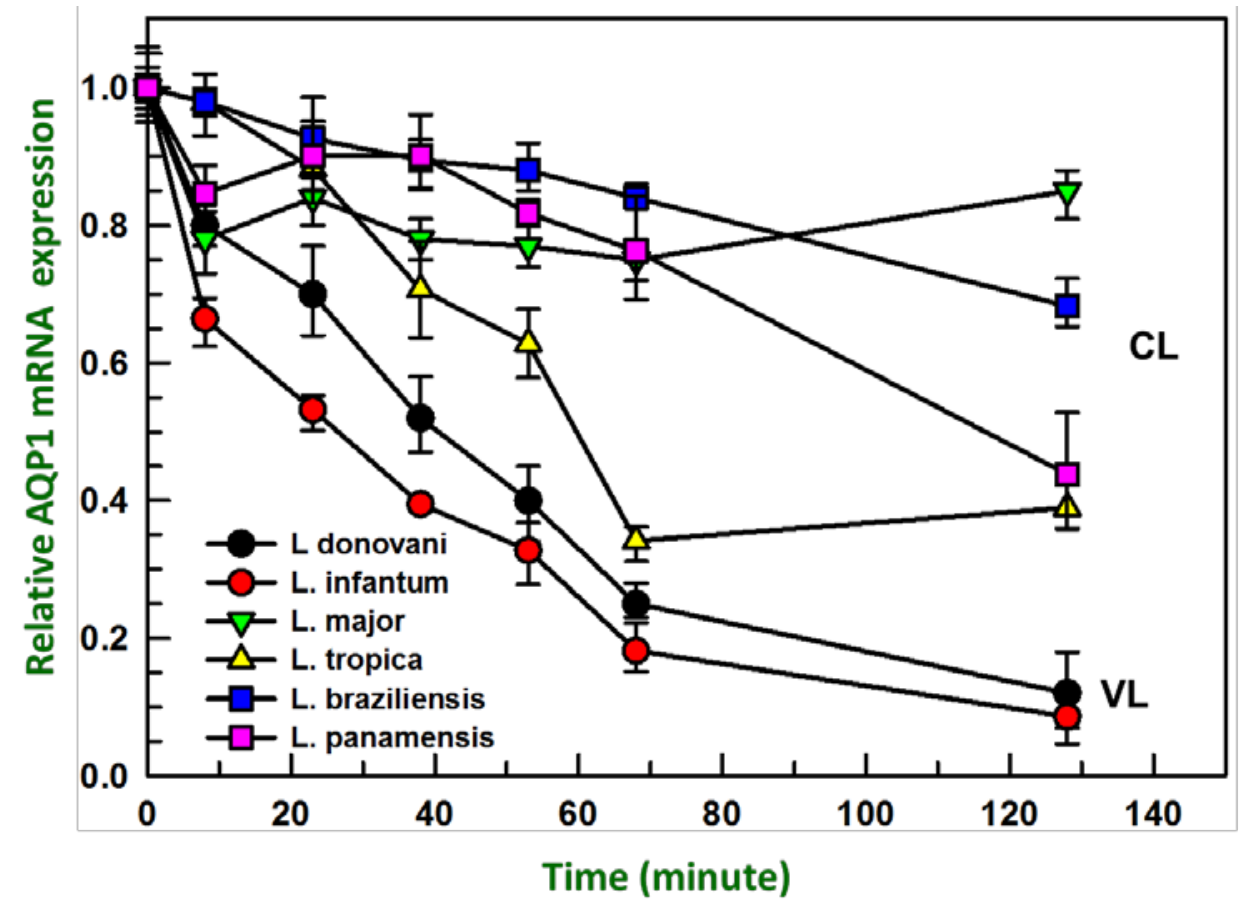

Figure 35 . Stability of AQP1 mRNA in promastigotes of different species of Leishmania. Promastigotes of different species of Leishmania were exposed to sinefungin followed by actinomycin d. Cells were harvested just before the exposure of sinefungin ( 0 minute) and followed by at different time point after the exposure of actinomycin D. Total RNA were isolated and AQP1 mRNA expression levels were estimated using QPCR. Relative (with respect to 0 minute) AQP1 expression levels were calculated using $2^{-\Delta \Delta C t}$ method. Data were expressed as mean \pm SD of three independent experiments. 


\subsubsection{Cloning of 3'-UTRs of AQP1 mRNA from different species}

In order to obtain a detailed insight into mRNA stability in all six different species of Leishmania, we cloned individual 3'-UTRs of AQP1 mRNA from the Cutaneous leishmaniasis and VL species described in this study (Mandal et al., 2015). Using the 3'RACE PCR technique, it was found that the length of the 3'-UTR of the AQP1 mRNA in each of the six species was approximately $1.8 \mathrm{~kb}$ in length. When we compared the protein alignment sequences of AQP1 for all the species in ClustalW, we observed an overall similarity of $88 \%$. Also, the overall identity was $66 \%$ between the open reading frames (ORF) of AQP1 in all six species. However, the sequences of the 3'-UTR from each of the six species were found to be more divergent and showed an overall identity of only $24 \%$. We found greater similarities between the pairs, for example, $99 \%$ identity between $L$. donovani and L. infantum (VL) 3'-UTR, 78\% identity between L. major and L. tropica and 94\% identity between $L$. braziliensis and $L$. panamensis.

\subsubsection{Role of the 3'-UTR in species-specific stability of AQP1 mRNA}

Because 3'-UTR sequences are divergent between VL and Cutaneous leishmaniasis species, we hypothesized that these sequences are responsible for the species-specific differential $A Q P 1$ mRNA stability. In order to understand the principal mechanism, we made a series of chimeric constructs with the full length 3'-UTR ( 1.8 $\mathrm{kb})$ of the AQP1 mRNA from each of the six species by cloning them at the 3' end of the luciferase (LUC) reporter gene (Figure 36). 


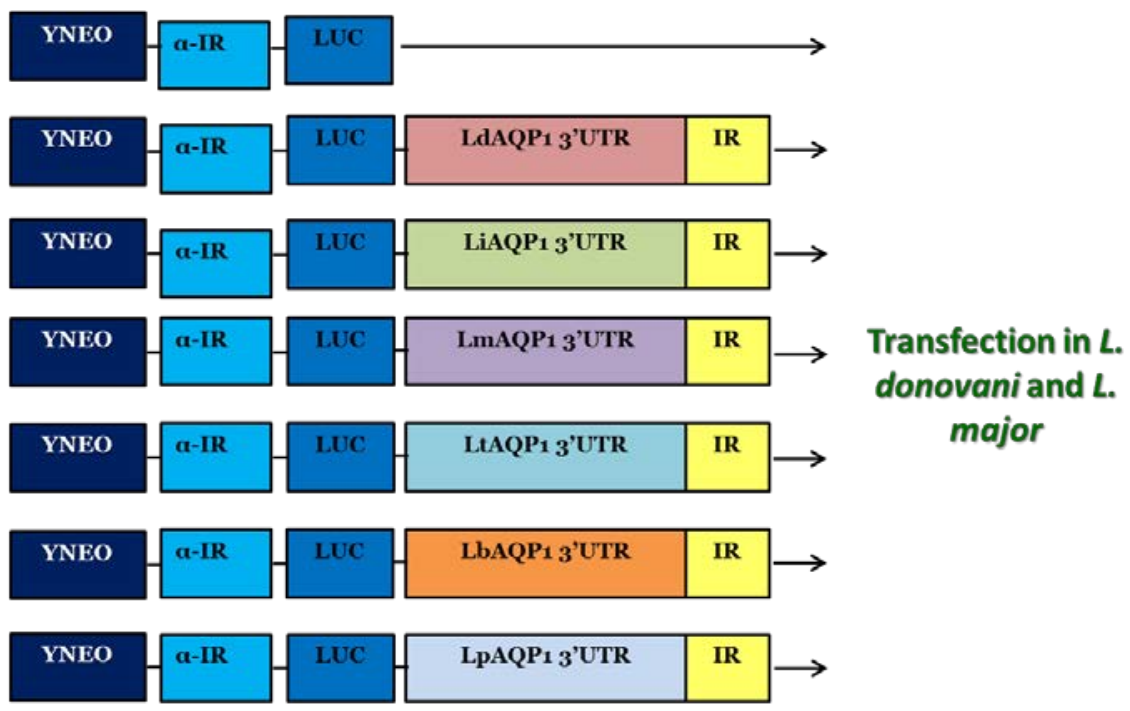

Figure 36. Exploring the role of 3'UTR in regulation of AQP1 using luciferase reporter system. AQP1 3'UTRs and intergenic regions from different species of Leishmania were cloned at the 3' end of LUC ORF of pSPYNEOaLUC vector. The neomycin resistance gene (NEO) and LUC transcripts in pSPYNEO $\alpha$ LUC vector are processed either by a 92 synthetic polypyrimidine stretch (Y90AG) (YNEO) or by the intergenic region of the $\alpha$-tubulin gene $(\alpha$-IR) respectively. The 3'-UTR $(\sim 1.8 \mathrm{~kb})$ plus a 200 bp downstream intergenic sequence of the AQP1 3'UTR (IR) from different species were PCR amplified from the genome of different species of Leishmania and introduced at the 3' end of the LUC gene. and transfected each constructs into L. donovani and L. major.

These cassettes were flanked by an upstream $\alpha$-tubulin intergenic (IR) region and by a downstream IR region ( $200 \mathrm{bp}$ ) of each species-specific 3'-UTR of AQP1 mRNA to maintain the 5' and 3' processing of the LUC chimeric transcripts accurately. Transsplicing signals, which are located 100-400 nt downstream of the polyadenylation site, often direct polyadenylation in trypanosomes (LeBowitz et al., 1993; Matthews et al., 1994; Boucher et al., 2002). These chimeras were expressed from an episomal plasmid pSPYNEO $\alpha$ LUC with neomycin phosphotransferase (NEO) as a marker. In the current study, pLUC referred to the vector alone, where $L U C$ expression was only regulated by the IR region of $\alpha$-tubulin. Each of the species-specific LUC-AQP1-3'-UTR constructs 
were named pLUC-Ld (L. donovani), pLUC-Li (L. infantum), pLUC-Lm (L. major), pLUC-Lt (L. tropica), pLUC-Lb (L. braziliensis), and pLUC-Lp (L. panamensis). Six individual chimeric constructs and the vector alone were transfected into the six species generating 42 transfectants (Mandal et al., 2015). However, our discussion will be limited to the role of six 3'-UTRs in regulating LUC protein levels and LUC activity in a species-specific manner in L. donovani and L. major, as both species have remarkable differences in their 3'-UTR AQP1 sequences. We will also address the mRNA steady state levels and stability in order to explain the role of all six 3'-UTRs in all six species. After L. donovani was transfected with each of the six different chimeric constructs and vector alone, we carried out western blot studies with an anti-luciferase antibody and completed densitometric analyses using $\alpha$-tubulin as a loading control. We observed that luciferase expression was lowest in pLUC-Li transfectants, at only about 21\%, which resulted in about 18\% luciferase activity when compared to pLUC control (Figure 37 and 38). pLUC-Ld, pLUC-Lm, pLUC-Lt, pLUC-Lb and pLUC-Lp transfectants expressed

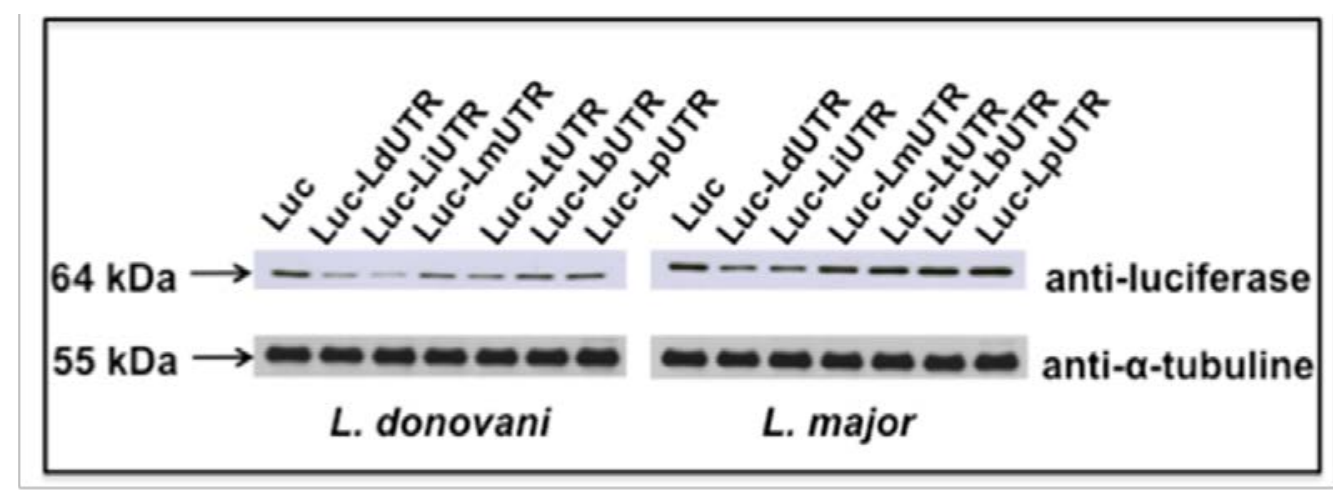

Figure 37. Western Blot analysis of transfected promastigotes. Whole cell $\left(1 \times 10^{6} /\right.$ lane $)$ lysates of different transfectants were fractionated on SDS-PAGE and blotted onto nitrocellulose membrane. Level of luciferase expressions were detected using anti-luciferase antibody and anti- $\alpha$-tubulin antibody. $\alpha$-tubulin was used as loading control. $L d-L$. donovani , Li- L. infantum, Lm- L. major, LtL. tropica, Lb- L. braziliensis, Lp- L. panamensis 
$1.5,4.5,2.6,5.5$, and 4.7 times more luciferase, respectively, when compared to pLUC-Li (Figure 38). Similarly, when all the constructs were transfected in L. major, LUC expressions were similar in all of the Cutaneous leishmaniasis UTR constructs (Figure 37). Further, $77 \%$ and $93 \%$ of LUC was expressed by pLUC-Li and pLUC-Ld transfectants, respectively, when compared to pLUC controls. The highest LUC activity was observed in cells expressing LUC from pLUC-Lm, followed by pLUC-Lp, pLUC-Lt and pLUC-Lb (Figure 38).

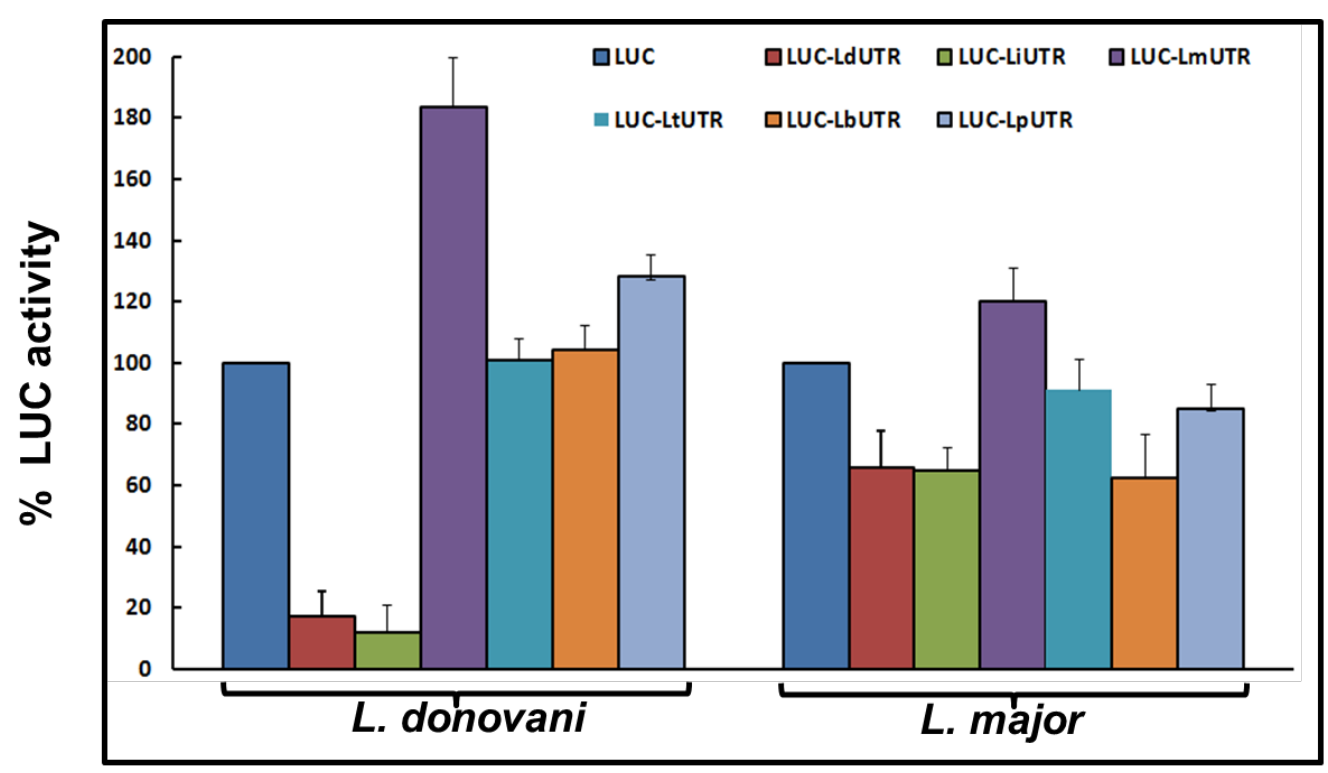

Figure 38. Effect of AQP1 3'-UTR from different species of Leishmania on luciferase (LUC) activity. LUC activity assay was carried out using cell lysates. Percent LUC activity was calculated keeping vector alone control at $100 \%$. Data were expressed as mean \pm SEM of three independent experiments in duplicates.

\subsection{Discussion}

There is no single effective treatment available for all forms of leishmaniasis (Gonzalez et al., 2009). Based on geographical location and the species responsible for infection, options for treatment are limited (Croft and Coombs 2003). The first line of 
treatment against all forms of leishmaniasis is an antimonial drug, but treatment failure is a major problem. Treatment of American cutaneous leishmaniasis (ACL) is very complex and challenging. Most commonly, intrinsic and acquired variations in the sensitivity of different Leishmania species are the factors, which influence the efficacy of drugs. Pentavalent antimonials are the most prescribed form of drugs for the treatment of ACL and MCL. As per World Health Organization (WHO) standards, the recommended dose of pentavalent antimonial for the treatment of ACL is $20 \mathrm{mg} / \mathrm{kg} / \mathrm{day}$ for 28 days (Reithinger et al., 2007). Local physicians in the respective geographical location determine appropriate antimonial drug dosage based on experience and cannot be standardized (Tuon et al., 2008). It is clinically proven that antimonial resistance prevails not only amongst different Leishmania species but it is also strain specific. All these factors create a major impediment in implementing a single dose strategy for all forms of leishmaniasis. Decuypere et al., 2012 reported that, under antimonial treatment pressure, molecular and phenotypic heterogeneities emerged in an L. donovani population of patients from Nepal. Several studies reported that each genetically diverse population could acquire an antimonial resistant phenotype with a different molecular basis. Nevertheless, not a single mechanism in L. donovani was identified. On the other hand, cutaneous leishmaniasis species have greater susceptibility towards oxidative stress because the production of non-protein thiols is much less when compared to the visceral leishmaniasis species from the Indian subcontinent (Sarkar et al., 2012). Mandal et al., 2015 reported that the non-protein thiol levels were similar in all VL and Cutaneous leishmaniasis species. Our study aimed to address the controversial issue of speciesspecific antimonial sensitivity in Leishmania. We examined all commonly used drug 
resistant markers ((i)MRPA (Legare et al., 2001); (ii) thiols (Mukhopadhyay et al., 1996; Mittal et al., 2007); (iii) an unknown efflux system (Dey et al., 1994; Dey et al., 1996); and (iv) AQP1 (Decuypere et al., 2005; Marquis et al., 2005; Maharjan et al., 2008) and determined whether there were any direct correlations among four Old World Leishmania species (L. donovani, L. infantum, L. tropica and L. major) and two New world species (L. panamenisis and L. braziliensis).

Among the four most commonly used antimonial resistance makers, our discussion is focused on two factors, an efflux system and AQP1, in each of the six species. MRPA and total non-protein thiol levels were addressed by Dr. Mandal, who observed that the MRPA mRNA levels were much higher in Cutaneous leishmaniasis species as compared to VL species. In his studies, the total non-protein thiol levels were similar in both the Cutaneous leishmaniasis and VL species. The Sb-[TS $]_{2}$ efflux pump rate was similar in all six species, whereas downregulation of AQP1 seemed to be driving the species-specific antimonial sensitivity. The two visceral species showed lower AQP1 mRNA levels, less Sb(III) accumulation and less osmoregulatory capacity when compared to cutaneous species (Mandal et al., 2015).

In this study, we proved that the species-specific antimonial sensitivity in Leishmania is being driven by the regulation of AQP1 at the mRNA level. We also showed that AQP1 mRNA is more stable in Cutaneous leishmaniasis species as compared to VL species. Since, Leishmania does not have any transcriptional control, role of AQP1 3'-UTRs in the species-specific stabilization of AQP1 mRNA was determined by using the luciferase reporter assay. Mandal et al., 2015 reported that, 
under the control of VL 3'-UTR, LUC mRNA accumulation was found to be less in both VL and Cutaneous leishmaniasis species. This proposes that there are no species-specific factors responsible for the regulation at the level of AQP1 mRNA stability. However, a difference in 3'-UTR sequences makes the trans-acting factor (s) more favorable to binding and to stabilizing or destabilizing the AQP1 mRNA, but there is no difference between the expression levels of 3'-UTRs of Cutaneous leishmaniasis species in VL and Cutaneous leishmaniasis species. This suggests that there is no effect from any speciesspecific trans-acting factor. Mandal et al., 2015 reported that all 3'-UTRs of AQP1 provided LUC mRNA with the highest level of stability in L. major.

It has been thoroughly investigated that multiple factors are responsible for mRNA stability in higher eukaryotes and mammalian cells. Perhaps the best characterized family of instability determinants is the A+U rich elements (ARE) in the 3'-UTR, which are known to regulate a variety of transcripts via mRNA degradation and/or translational repression (Knapinska et al., 2011). Some known AUBPs (A + U binding proteins) are AUF1/hnRNP (heterogeneous nuclear ribonucleoprotein), TTP, BRF1 (Butyrate response factor), KSRP (KH-type splicing regulatory protein), TIA-1 (Tcell internal antigen 1), and TIAR (TIA-1 related protein) (Barreau et al., 2005). In trypanosomatids, RNA-binding proteins (RBP) are key factors in regulating gene expression. Over one hundred RBPs have been predicted to exist in trypanosomes, based on the presence of canonical RNA-binding domains: RNA recognition motif (RRM), $\mathrm{CCCH}$ zinc fingers, and Pumilio or PUF domains. Most of the characterized RBPs control mRNA abundance by stabilizing the target transcript. In trypanosomatids, although indirect results suggest that some proteins cause degradation of target 
transcripts, this has never been shown directly (Erben et al., 2014). Since AQP1 mRNA in the VL species is more unstable compared to the Cutaneous leishmaniasis species, and this phenomenon is driven by their respective 3'-UTRs, we analyzed the predicted 3'UTR RNA structures of the most resistant (L. infantum) and the most sensitive ( $L$. major), using RNA structure folding programs such as RNA fold and REPFIND. We found two major AU-rich regions in L. infantum 3'-UTR sequence, whereas L. major had only one (Figure 39A and B).

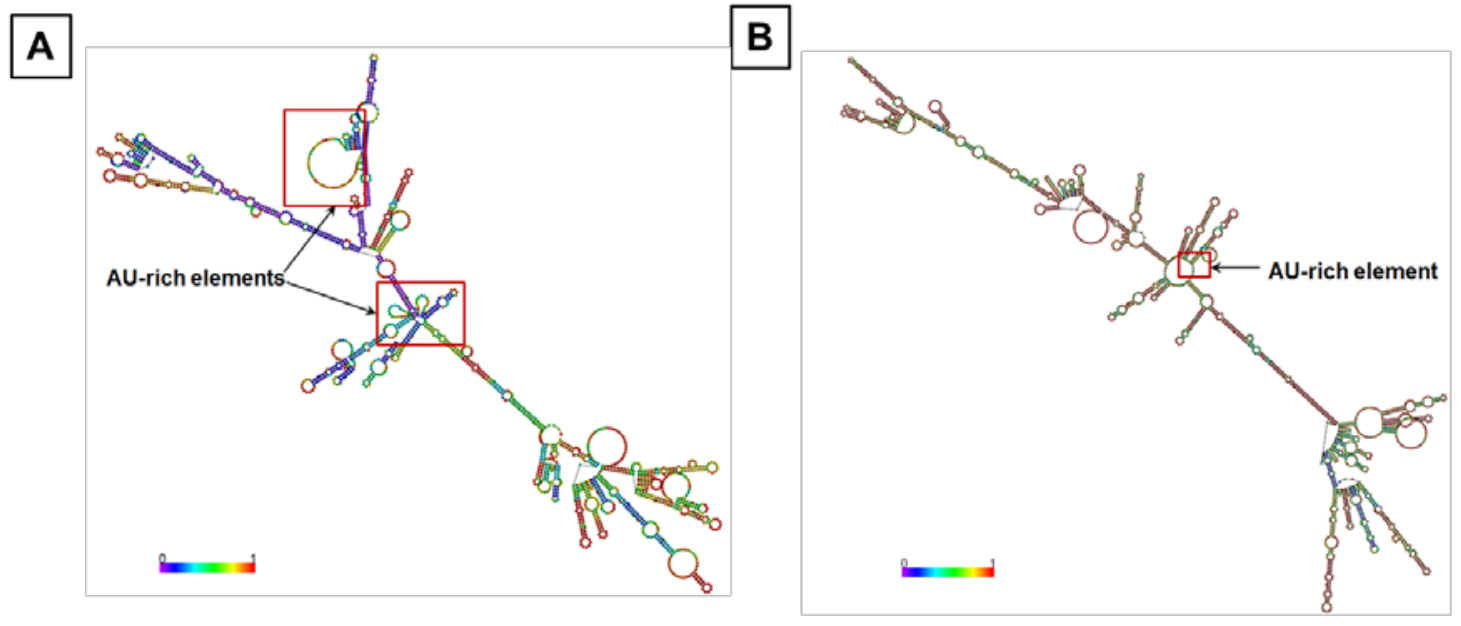

Figure 39. Secondary structure prediction of AQP1 3'-UTR (A) L. infantum and (B) L. major based on Minimum Free Energy (MFE) by RNAfold web Server. Arrows indicate ARE regions. The base-pair probabilities are indicated in scale by different colors. 
Secondary structure analyses showed that both of these regions are present in single-stranded loops in two out of three predictions (Figure 40) in L. infantum, while in L. major, they are in hairpins in all three predictions (Figure 40). It has been observed that destabilizing elements preferably bind to ARE regions when they are present in loop structures (Meisner et al., 2004). Also, CA repeats in the 3'-UTR have been reported to mediate constitutive decay, as is the case of bcl2 mRNA (Lee et al., 2004). There are multiple CA repeats present in the AQP1 3' UTRs of all species with the longest being in L. infantum (Figure 39). Therefore, it is intriguing to speculate that these VL specific motifs may play a role in the instability of the $A Q P 1$ mRNA. However, there may be other specific regions that are involved and yet not so evident from the primary sequences. Therefore, a systematic study of the 3'-UTRs and trans-acting factors is necessary, which is currently in progress.

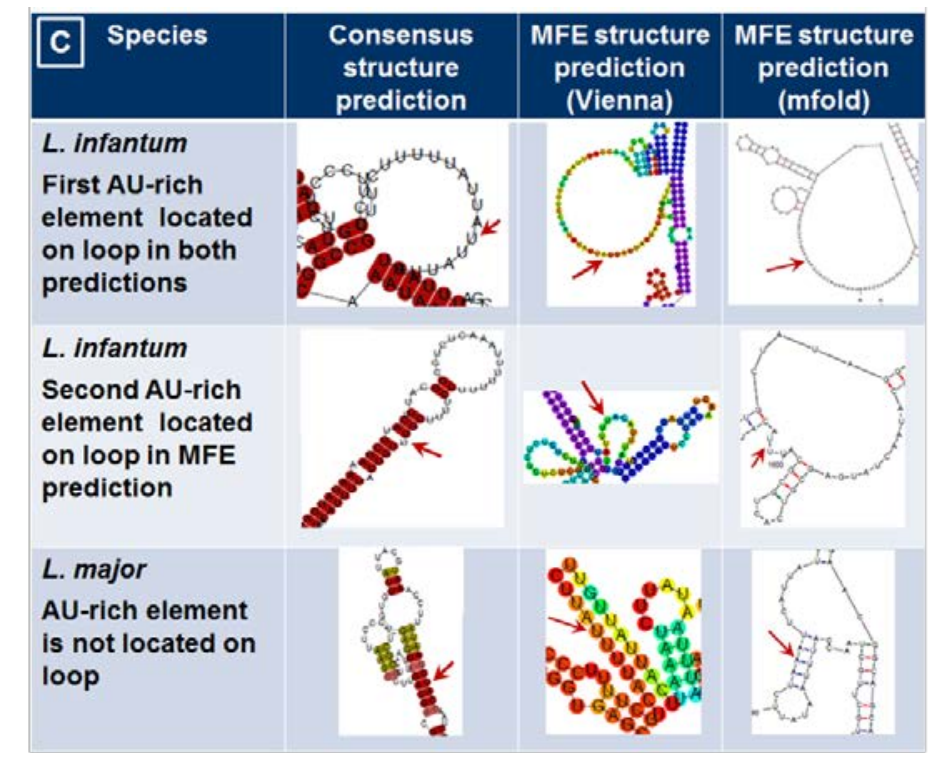

Figure 40. Secondary structure prediction of $A Q P 1$ 3'-UTR. Highlighted ARE regions from L. infantum and $L$. major by different platforms predictions: LocARNA (consensus structure), Vienna and mfold (MFE). The arrows indicate ARE regions. 
The most intriguing question is why there is a species-specific regulation of AQP1 in Leishmania. Antimonial resistance in Leishmania can be the result of a variety of factors. It can be speculated that VL species show a higher antimonial resistance due to having a higher intrinsic mRNA instability, which in turn leads to a lower production of AQP1 (Haldar et al., 2011). Conversely, the Cutaneous leishmaniasis species may be more efficient osmoregulators because it faces greater osmotic challenges during vector to host transmission, and more AQP1 may help them to overcome the barrier. AQPs sometimes play an important role in other physiological processes and functions, such as lipid metabolism, epidermal biology, cell adhesion and, neural signal transduction (Mukhopadhyay et al., 2014). So, it is very interesting to speculate that species-specific AQP1 expression may help the respective species to find their appropriate niches, resulting in tissue tropism. In the cases of VL and Cutaneous leishmaniasis treatments, the amount of prescribed medication is similar and standardized by WHO. So, it is quite possible that the lower dosage of antimonials for Kala-azar may have been the reason for the more prevalent drug resistance phenotype in the VL species of the field. These are ambitious and yet intriguing and fundamental questions in Leishmania biology, which will be advanced by our novel finding of 3'-UTR driven species-specific regulation of AQP1. 


\subsection{References}

Aly, R., Argaman, M., Halman, S., \& Shapira, M. (1994). A regulatory role for the 5' and 3' untranslated regions in differential expression of hsp83 in Leishmania. Nucleic Acids Research, 22(15), 2922-2929.

Barreau, C., Paillard, L., \& Osborne, H. B. (2005). AU-rich elements and associated factors: are there unifying principles? Nucleic Acids Research, 33(22), 7138-7150.

Beetham, J. K., Myung, K. S., McCoy, J. J., Wilson, M. E., \& Donelson, J. E. (1997). Glycoprotein 46 mRNA abundance is post-transcriptionally regulated during development of Leishmania chagasi promastigotes to an infectious form. The Journal of Biological Chemistry, 272(28), 17360-17366.

Boucher, N., Wu, Y., Dumas, C., Dube, M., Sereno, D., Breton, M., et al. (2002). A common mechanism of stage-regulated gene expression in Leishmania mediated by a conserved 3'-untranslated region element. The Journal of Biological Chemistry, 277(22), 19511-19520.

Bringaud, F., Muller, M., Cerqueira, G. C., Smith, M., Rochette, A., El-Sayed, N. M., et al. (2007). Members of a large retroposon family are determinants of posttranscriptional gene expression in Leishmania. PLoS Pathogens, 3(9), 1291-1307.

Croft, S. L., \& Coombs, G. H. (2003). Leishmaniasis-current chemotherapy and recent advances in the search for novel drugs. Trends in parasitology, 19(11), 502-508.

Erben, E. D., Fadda, A., Lueong, S., Hoheisel, J. r. D., \& Clayton, C. (2014). A genomewide tethering screen reveals novel potential post-transcriptional regulators in Trypanosoma brucei. PLoS Pathogens, 10(6), e1004178.

Figarella, K., Uzcategui, N. L., Zhou, Y., Lefurgey, A., Ouellette, M., Bhattacharjee, H., et al. (2007). Biochemical characterization of Leishmania major aquaglyceroporin LmAQP1: possible role in volume regulation and osmotaxis. Molecular Microbiology, 65(4), 1006-1017.

Frezard, F., Demicheli, C., \& Ribeiro, R. R. (2009). Pentavalent antimonials: new perspectives for old drugs. Molecules, 14(7), 2317-2336.

Gourbal, B., Sonuc, N., Bhattacharjee, H., Legare, D., Sundar, S., Ouellette, M., et al. (2004). Drug uptake and modulation of drug resistance in Leishmania by an aquaglyceroporin. The Journal of Biological Chemistry, 279(30), 31010-31017.

Haile, S., \& Papadopoulou, B. (2007). Developmental regulation of gene expression in trypanosomatid parasitic protozoa. Current Opinion in Microbiology, 10(6), 569577. 
Haldar, A. K., Sen, P., \& Roy, S. (2011). Use of antimony in the treatment of leishmaniasis: current status and future directions. Molecular Biology International, 2011, 571242.

Knapinska, A. M., Gratacòs, F. M., Krause, C. D., Hernandez, K., Jensen, A. G., Bradley, J. J., et al. (2011). Chaperone Hsp27 Modulates AUF1 Proteolysis and AU-Rich Element-Mediated mRNA Degradation. Molecular and Cellular Biology, 31(7), 1419-1431.

Kramer, S. (2012) Developmental regulation of gene expression in the absence of transcriptional control: the case of kinetoplastids. Molecular and Biochemical Parasitology, 181(2), 61-72.

Lamontagne, J., \& Papadopoulou, B. (1999). Developmental regulation of spliced leader RNA gene in Leishmania donovani amastigotes is mediated by specific polyadenylation. The Journal of Biological Chemistry, 274(10), 6602-6609.

LeBowitz, J. H., Smith, H. Q., Rusche, L., \& Beverley, S. M. (1993). Coupling of poly(A) site selection and trans-splicing in Leishmania. Genes and Development, 7(6), 996-1007.

Lee, J. H., Jeon, M. H., Seo, Y. J., Lee, Y. J., Ko, J. H., Tsujimoto, Y., et al. (2004). CA Repeats in the 3'-Untranslated Region of bcl-2 mRNA mediate constitutive Decay of bcl-2 mRNA. The Journal of Biological Chemistry, 279(41), 42758-42764.

Legare, D., Papadopoulou, B., Roy, G., Mukhopadhyay, R., Haimeur, A., Dey, S., et al. (1997). Efflux systems and increased trypanothione levels in arsenite-resistant Leishmania. Experimental Parasitology, 87(3), 275-282.

Mandal, G., Mandal, S., Sharma, M., Charret, K. S., Papadopoulou, B., Bhattacharjee, H., et al. (2015). Species-specific antimonial sensitivity in Leishmania is driven by post-transcriptional regulation of AQP1. PLoS Neglected Tropical Diseases, 9(2), e0003500.

Mandal, G., Orta, J., Sharma, M., \& Mukhopadhyay, R. (2014). Trypanosomatid aquaporins: Roles in physiology and drug response. Diseases, 2(1), 3.

Mandal, S., Maharjan, M., Singh, S., Chatterjee, M., \& Madhubala, R. (2010). Assessing aquaglyceroporin gene status and expression profile in antimony-susceptible and resistant clinical isolates of Leishmania donovani from India. Journal of Antimicrobial Chemotherapy, 65(3), 496-507.

Marquis, N., Gourbal, B., Rosen, B. P., Mukhopadhyay, R., \& Ouellette, M. (2005). Modulation in aquaglyceroporin $A Q P 1$ gene transcript levels in drug-resistant Leishmania. Molecular Microbiology, 57(6), 1690-1699. 
Matthews, K. R., Tschudi, C., \& Ullu, E. (1994). A common pyrimidine-rich motif governs trans-splicing and polyadenylation of tubulin polycistronic pre-mRNA in trypanosomes. Genes and Development, 8(4), 491-501.

Mayer, M. G., Santos, M. G., Silva, M. F., \& Floeter-Winter, L. M. (2012) Footprints of a trypanosomatid RNA world: pre-small subunit rRNA processing by spliced leader addition trans-splicing. Memorias do Instituto Oswaldo Cruz, 107(4), 522531.

McNicoll, F., Muller, M., Cloutier, S., Boilard, N., Rochette, A., Dube, M., et al. (2005). Distinct 3'-untranslated region elements regulate stage-specific mRNA accumulation and translation in Leishmania. The Journal of Biological Chemistry, 280(42), 35238-35246.

Meisner, N. C., Hackermüller, J., Uhl, V., Aszódi, A., Jaritz, M., \& Auer, M. (2004). mRNA Openers and Closers: Modulating AU-Rich Element-Controlled mRNA Stability by a Molecular Switch in mRNA Secondary Structure. ChemBioChem, 5(10), 1432-1447.

Mishra, K. K., Holzer, T. R., Moore, L. L., \& LeBowitz, J. H. (2003). A negative regulatory element controls mRNA abundance of the Leishmania mexicana paraflagellar rod gene PFR2. Eukaryotic Cell, 2(5), 1009-1017.

Mukhopadhyay, R., Bhattacharjee, H., \& Rosen, B. P. (2014). Aquaglyceroporins: generalized metalloid channels. Biochimica et Biophysica Acta, 1840(5), 15831591.

Mukhopadhyay, R., Dey, S., Xu, N., Gage, D., Lightbody, J., Ouellette, M., et al. (1996). Trypanothione overproduction and resistance to antimonials and arsenicals in Leishmania. Proceedings of the National Academy of Sciences, 93(19), 1038310387.

Muller, M., Padmanabhan, P. K., Rochette, A., Mukherjee, D., Smith, M., Dumas, C., et al.(2010). Rapid decay of unstable Leishmania mRNAs bearing a conserved retroposon signature 3'-UTR motif is initiated by a site-specific endonucleolytic cleavage without prior deadenylation. Nucleic Acids Research, 38(17), 58675883.

Myung, K. S., Beetham, J. K., Wilson, M. E., \& Donelson, J. E. (2002). Comparison of the post-transcriptional regulation of the mRNAs for the surface proteins PSA (GP46) and MSP (GP63) of Leishmania chagasi. The Journal of Biological Chemistry, 277(19), 16489-16497.

Ramamoorthy, R., Swihart, K. G., McCoy, J. J., Wilson, M. E., \& Donelson, J. E. (1995). Intergenic regions between tandem gp63 genes influence the differential 
expression of gp63 RNAs in Leishmania chagasi promastigotes. The Journal of Biological Chemistry, 270(20), 12133-12139.

Reithinger, R., Dujardin, J. C., Louzir, H., Pirmez, C., Alexander, B., \& Brooker, S. (2007). Cutaneous leishmaniasis. The Lancet Infectious Diseases, 7(9), 581-596.

Sarkar, A., Ghosh, S., Pakrashi, S., Roy, D., Sen, S., \& Chatterjee, M. (2012). Leishmania strains causing self-healing cutaneous leishmaniasis have greater susceptibility towards oxidative stress. Free Radical Research, 46(5), 665-673.

Schaffner, W., \& Weissmann, C. (1973). A rapid, sensitive, and specific method for the determination of protein in dilute solution. Analytical Biochemistry, 56(2), 502514.

Sharma, M., Mandal, G., Mandal, S., Bhattacharjee, H., \& Mukhopadhyay, R. (2015). Functional role of lysine 12 in Leishmania major AQP1. Molecular and Biochemical Parasitology, 201(2), 139-145.

Tuon, F. F., Amato, V. S., Graf, M. E., Siqueira, A. M., Nicodemo, A. C., \& Amato Neto, V. (2008). Treatment of New World cutaneous leishmaniasis-a systematic review with a meta-analysis. International Journal of Dermatology, 47(2), 109-124.

Uzcategui, N. L., Zhou, Y., Figarella, K., Ye, J., Mukhopadhyay, R., \& Bhattacharjee, H. (2008). Alteration in glycerol and metalloid permeability by a single mutation in the extracellular C-loop of Leishmania major aquaglyceroporin LmAQP1. Molecular Microbiology, 70(6), 1477-1486.

Zhou, Y., Bhattacharjee, H., \& Mukhopadhyay, R. (2006). Bifunctional role of the leishmanial antimonate reductase LmACR2 as a protein tyrosine phosphatase. Molecular and Biochemical Parasitology, 148(2), 161-168. 
CHAPTER 4

\section{CONCLUSIONS AND FUTURE DIRECTIONS}


Water is the chief component of all living cells. Regulation of water homeostasis is indispensable for many biological processes. A persistent question for many years was how water passed through biological membranes. It was Peter Agre and his co-workers, in 1992 that identified the first mammalian water-selective channel in erythrocytes known as aquaporins or AQPs. Significant advances have been made with regard to mammalian AQPs. Mammalian AQPs are known to be involved in several diseases such as congenital cataracts, diabetes insipidus (NDI), brain edema, obesity, liver steatosis and type II diabetes mellitus (Gena et al., 2011). In several studies, AQPs are the potential drug targets for drug synthesis or genetic engineering strategies to cure and/or prevent human diseases (Gena et al., 2011). However, knowledge about parasite AQPs is lagging far behind. Parasite AQPs are essential for the successful prolongation of the diseases caused by them. Exploration of regulatory mechanisms of parasite AQPs will reveal their potential for novel chemotherapeutic approaches and/or transmission intervention(s) (Mandal et al., 2014). As discussed in chapter I, aquaporins belong to the Major Intrinsic Proteins (MIP) superfamily, and depending on the substrate specificity, aquaporins can be divided into groups: “Aquaporins”, which facilitate the movement of water only, and "aquaglyceroporins", which facilitate the movement of water along with glycerol and other small uncharged solutes.

Aquaglyceroporins are instrumental in the transport of metalloids. Metalloids, such as arsenic and antimony, are the intermediate elements which have properties of both metals and non-metals. Arsenic and antimony cause toxicity, but also have chemotherapeutic values in treating medical conditions such as acute promyelocytic 
leukemia (Beitz 2006) and several protozoan diseases. Aquaglyceroporins facilitate the uptake of Arsenic(As) and Antimony(Sb), metalloids that can exist in pentavalent and trivalent oxidation state (Mukhopadhyay and Beitz 2010). At neutral $\mathrm{pH}$, the trivalent forms of these metalloids assume a molecular structure similar to glycerol; hence, they enter inside the cells through AQPs. In E.coli, GlpF acts as a major route of As (III) entry (Bhattacharjee et al., 2008; Rosen and Tamás 2010). Similarly, hAQP9 and plant NIPs are able to conduct metalloids across the membrane (Bhattacharjee et al., 2008). In Saccharomyces cerevisiae, Fps1p aquaglyceroporin acts as As(III) transporter (Figure 41) (Liu et al., 2002).

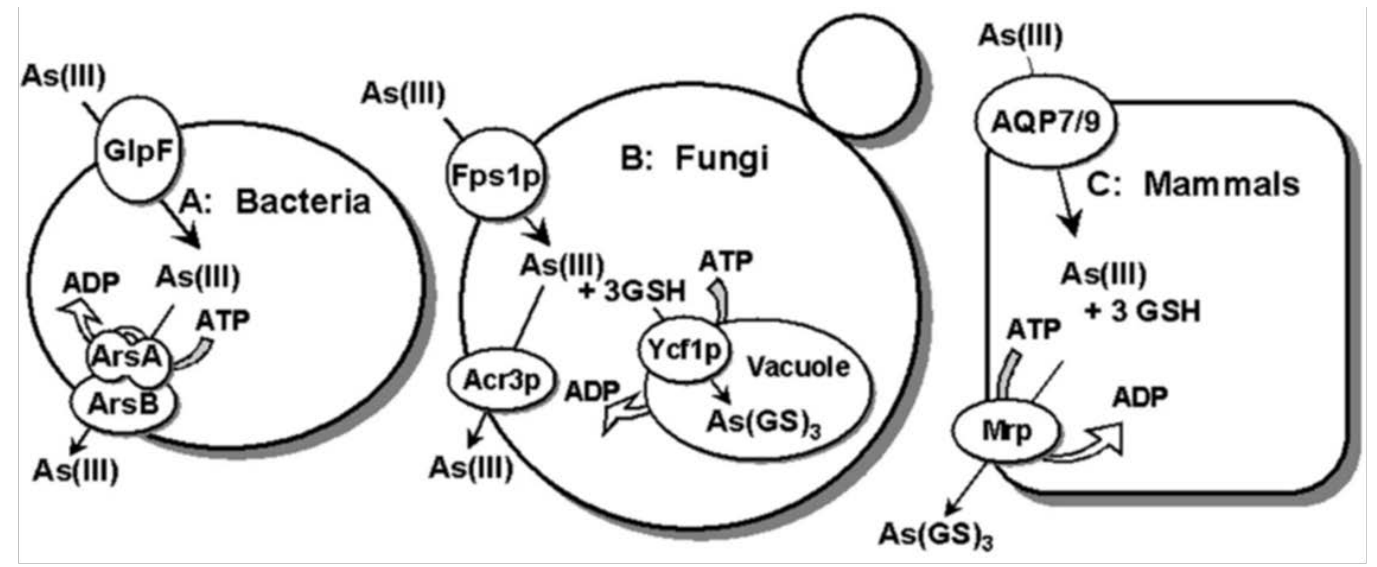

Figure 41. Metalloid transporters in prokaryotes and eukaryotes. The model shows a comparison of arsenite transport pathways in $E$. coli, S. cerevisiae and a generalized mammalian cell . In each of three, arsenite uptake is facilitated by an aquaglyceroporin: the bacterial GIpF, the yeast Fps1p, or the mammalian AQP7 or AQP9 (Liu et al. 2002)

As discussed in Chapter I, pentavalent antimonials $[\mathrm{Sb}(\mathrm{V})]$ are the primary drugs of choice against all forms of leishmaniasis (Gourbal et al., 2004). Sb(V), a prodrug, is reduced to trivalent antimony [Sb(III)], which is the active form of the drug. Sadly, 
several cases of leishmaniasis are becoming unresponsive to antimonials. Options for second line of treatments are very limited and/or expensive and/or more toxic. Thus, to modulate the drug unresponsiveness, there is a dire need for increased understanding of the antimony resistance mechanisms in Leishmania. Antimony resistance in Leishmania is multifactorial (Mandal et al., 2015). One of the important mechanisms is down regulation of the $\mathrm{Sb}(\mathrm{III})$ facilitator. Our research group was the first to demonstrate the existence of a Sb(III) facilitator in Leishmania, namely, aquaglyceroporin 1 (AQP1). AQP1 is an adventitious facilitator of $\mathrm{Sb}$ (III) and to date the only reported facilitator of $\mathrm{Sb}(\mathrm{III})$ in Leishmania. In Leishmania, AQP1 is involved in several important physiological functions, including osmoregulation and osmotaxis (Figarella et al., 2007). Efficient osmoregulation and osmotaxis are necessary for Leishmania, as the parasites face diverse osmotic challenges during the life cycle. Hence, AQP1 is essential for the survival of promastigotes. To conquer these challenges, Leishmania needs to regulate AQP1 efficiently. Taken together, delineation of the AQP1 regulatory network could provide the tools for better management of this deadly pathogen. Accordingly, the primary objective of my dissertation research was to understand the regulatory mechanism of AQP1 in Leishmania. Expression of AQP1 in Leishmania is highly regulated. It is known that, in the absence of definitive promoters, Leishmania primarily regulates its genes at post-transcriptional and/or post-translational levels. We reported that mitogen activated protein kinase 2 (MPK2) positively regulated AQP1 stability through phosphorylation of the Thr197 residue (Mandal et al., 2012). AQP1 phosphorylated at Thr197 showed more stability whereas the absence of phosphorylation 
makes AQP1 more susceptible to degradation. It was also observed that in the presence of a variant MPK2 (MPK $\left.{ }_{\text {42A }}\right)$ AQP1 was subjected to accelerated destabilization.

Based on these studies, I investigated the role of ubiquitin mediated posttranslational regulation of Leishmania AQP1- an unknown mechanism for AQPs in protozoan parasites. I analyzed the sequence of AQP1 using UbPred and RONN, and identified $\mathrm{K} 12$ as the single putative ubiquitination site at the disordered N-terminus. When I treated the promastigotes with proteasomal inhibitor MG132, the cells became hypersensitive to MG132, and AQP1 was upregulated. When total membrane protein was isolated from MG132 treated cells, AQP1 expression was observed by immunoblot (Figure 19A) whereas the untreated cells did not show any expression of AQP1. When I altered K12 to alanine/arginine of AQP1 and co-expressed it with MPK2 $2_{42 \mathrm{~A}}$, the protein showed stability by increasing their half-lives (Figure 28). Removal of the critical lysine also led to an increase in antimonial sensitivity of the promastigotes because the strains accumulated more of the drug (Table 3). Previously, we reported that when co-expressed with MPK2, AQP1 was redistributed to the pellicular membrane along with the flagellar membrane but AQP1 maintained its flagellar localization in $\mathrm{AQP} 1 \mathrm{~K} 12 \mathrm{~A} / \mathrm{AQP} 1 \mathrm{~K} 12 \mathrm{R}+\mathrm{MPK} 2 \mathrm{wT}$ or $\mathrm{AQP} 1 \mathrm{~K} 12 \mathrm{~A} / \mathrm{AQP} 1 \mathrm{~K} 12 \mathrm{R}+\mathrm{MPK} 42 \mathrm{~A}$ promastigotes, in spite of the fact that AQP1 was highly stabilized with a very low turnover number. Based on the data, I proposed a model to describe a mechanism of AQP1 regulation at the post-translational level by probable ubiquitination2 and interaction with MPK2 at the disordered N-terminus of AQP1 (Figure 29). Results of this study proved that MPK2 $442 \mathrm{~A}$ mediated accelerated destabilization of AQP1 was due to the presence of a critical lysine 
12 (K12) at the disordered cytosolic N-terminus of the protein. While AQP1 is localized to the flagella of promastigotes, upon phosphorylation, it is distributed over the entire surface of the parasite. Absence of phosphorylation of AQP1 restricted its localization to flagellum only (Mandal et al., 2012). Interestingly, while MPK2 upregulated the expression of either $\mathrm{AQP} 1_{\mathrm{K} 12 \mathrm{~A}}$ or $\mathrm{AQP} 1_{\mathrm{K} 12 \mathrm{R}}$, the altered protein was localized to flagellum only. I interpreted this observation as lysine 12 being critical to pellicular localization of AQP1. The mechanisms for targeting proteins to flagella are now just being defined and are crucial to our understanding of flagellar function in kinetoplastids. In trypanosomes, a consensus tripeptide motif histidine-leucine-alanine (HLA) contained within two cytoskeletal flagellar proteins, paraflagellar rod A and actin-related protein, represents an essential part of a flagellar targeting machinery (Ersfeld and Gull 2001). However, such a feature is not universal. For example, the Leishmania enriettii glucose transporter, ISO1, resides primarily in the flagellar membrane, whereas the ISO2 glucose transporter is located in the pellicular plasma membrane. Deletion analyses suggest that the flagellar targeting information for ISO1 does not constitute a simple linear sequence of amino acids but a minimum of two separate sequences in tandem (Nasser and Landfear 2004). While no such sequence has been identified in Leishmania AQP1, our current observation leads us to speculate that, the N-terminus cytosolic region along with residues in loop D may be involved in targeting AQP1 between various cellular membranes. In the absence of transcriptional control, such a post-translational regulatory mechanism provides a novel way for Leishmania to survive during its digenetic life cycle. It is tempting to speculate that MPK2-mediated upregulation of AQP1 may counter the osmotic stress, which is critical for survival and transmission of the parasite 
between two environments, the vector and the vertebrate host. On the other hand, the intrinsically disordered $\mathrm{N}$-terminal domain containing lysine12 may serve as an on-off switch for proteasomal-dependent degradation, especially during drug exposure, thereby conferring resistance.

In addition, I studied the role of 3'-UTR in species-specific stability of AQP1 mRNA in Leishmania. As described in previous sections, due to the absence of promoters, protein coding genes are constitutively transcribed from large gene clusters as polycistronic pre-mRNAs in Leishmania. Most of the post-transcriptional gene regulation mechanism studies performed to date is based on stage specific gene expression in Leishmania, which involves 3'UTR elements that mediate the regulation at the level of mRNA abundance and translation, respectively. In this study, I worked with six different species of Leishmania: three cutaneous (L. major, L. tropica and $L$. panamensis), one mucocutaneous (L. braziliensis) and two visceral (L.donovani and L.infantum). When promastigotes (all six species) were exposed to increasing concentrations of potassium antimonyl tartarate, I observed that visceral species were more resistant to $\mathrm{Sb}(\mathrm{III})$ as compared to cutaneous species (Table 4) whereas $\mathrm{Sb}(\mathrm{III})$ accumulation was found to be much higher in Cutaneous leishmaniasis species (Figure 32). As Sb(III) accumulation was higher in Cutaneous leishmaniasis species, I measured the total non-protein thiol levels, which are markers for antimonial resistance, and found them to be similar in all of the species (Figure 33). When I exposed the promastigote cells to hypo-osmotic medium in order to measure osmoregulation, decreased levels of AQP1 levels were observed in VL species, and their osmoregulatory capacities were 
much less compared to Cutaneous leishmaniasis species (Figure 34). To determine whether the species-specific antimonial sensitivity in Leishmania is driven by regulation at the mRNA level, I treated the promastigote cells with sinefungin followed by actinomycin D. I observed that $A Q P 1$ mRNA was highly unstable in VL species as compared to the Cutaneous leishmaniasis species (Figure 35). In the absence of transcriptional control in Leishmania, the role of AQP1 3'-UTRs was determined in the species-specific stabilization of AQP1 using the luciferase reporter assay. 3'-UTRs from each of the six species were cloned. It was found that Cutaneous leishmaniasis 3'-UTRs of AQP1 mRNA were similarly stable in VL species as they were in Cutaneous leishmaniasis species, which implies that there is no effect from any species-specific trans-acting factors. Therefore, this study is highly significant. We have shown for the first time that species-specific antimonial sensitivity in Leishmania is uniquely driven by AQP1, and it is mediated by post-transcriptional regulation through the respective 3'UTR of each species-specific AQP1 mRNA.

This study has contributed to our understanding of the regulatory mechanism(s) of the drug facilitator AQP1, which has significant roles in the physiology of the parasite Leishmania as well as in its clinical resistance to antimonial drugs, the first lines of treatment against all forms of leishmaniasis. Leishmania AQP1 is novel in the AQP field because of its unique localization and its role in osmotaxis. My work established the critical role that AQP1 plays in the signal transduction interface of the parasite and its environment and that it interacts with other cellular molecules at the post-translational and post-transcriptional levels. These findings address a major gap in our understanding 
of Leishmania biology at the molecular level. Overall, Leishmania AQP1 bears about 30\% similarity to mammalian AQP9, which might make it difficult (but not impossible) to develop inhibitors targeted to AQP1. Conversely, unique regulators of AQP1 could be reasonable targets. For example, since protozoan MAP kinases are distantly related to human MAP kinases, it may be possible to design compounds that specifically target the protozoan enzymes. One approach may be the development of specific small molecule inhibitors of Leishmania MAPK2 activity. Such compounds may prevent phosphorylation of the protozoan AQP1 channel, resulting in increased turnover, possibly killing the parasites through osmotic stress. Another approach may be to identify small molecule inducers of MPK2 that might stabilize AQP1 and either reverse drug resistance or reduce drug toxicity by reducing the effective dose.

Drug resistance is a major impediment in treating leishmaniasis. To date, the species-specific antimony resistance had not been explored. My data supports the concept that treating all types of leishmaniasis with the same systemic dosage of antimony may not be the best practice. A lower concentration for the visceral infection (which may be the correct dose for the cutaneous species as they are more sensitive) may lead to the emergence of a more drug-resistant phenotype in that species. The results of this study may be relevant to the broader field of infectious diseases. Most physicians and scientists treat related species of a specific pathogen in similar ways. However, I propose that there may be differences in interspecies drug resistance within a single genus. Protozoan parasites such as Leishmania that depend on post-transcriptional and/or post-translational regulation, rather than transcriptional control, utilize one of these single 
mechanisms to control species-specific drug resistance. AQP1 is an adventitious antimonite facilitator in Leishmania. In addition, the osmoregulation in the host and osmotaxis in the vector are dependent on AQP1. Tissue tropism in Leishmania is species-specific. If species-specific regulation of AQP1 is at play in Leishmania, it is tempting to speculate that it may be involved in species-specific tissue tropism. The mechanism(s) of tissue tropism is one of the holy grails of Leishmania research. Thus, the results of this study may open up significant research in that direction. 


\subsection{References}

Beitz, E. (2006). Aquaporin water and solute channels from malaria parasites and other pathogenic protozoa. ChemMedChem, 1(6), 587-592.

Bhattacharjee, H., Mukhopadhyay, R., Thiyagarajan, S., \& Rosen, B. P. (2008). Aquaglyceroporins: ancient channels for metalloids. Journal of Biology, 7(9), 3333.

Ersfeld, K., \& Gull, K. (2001). Targeting of cytoskeletal proteins to the flagellum of Trypanosoma brucei. Journal of Cell Science, 114(1), 141-148.

Figarella, K., Uzcategui, N. L., Zhou, Y., LeFurgey, A., Ouellette, M., Bhattacharjee, H., et al. (2007). Biochemical characterization of Leishmania major aquaglyceroporin LmAQP1: possible role in volume regulation and osmotaxis. Molecular Microbiology, 65(4), 1006-1017.

Gena, P., Pellegrini-Calace, M., Biasco, A., Svelto, M., \& Calamita, G. (2011). Aquaporin membrane channels: Biophysics, classification, functions, and possible biotechnological applications. Food Biophysics, 6(2), 241-249.

Gourbal, B., Sonuc, N., Bhattacharjee, H., Legare, D., Sundar, S., Ouellette, M., et al. (2004). Drug uptake and modulation of drug resistance in Leishmania by an aquaglyceroporin. The Journal of Biological Chemistry, 279(30), 31010-31017.

Liu, Z., Shen, J., Carbrey, J. M., Mukhopadhyay, R., Agre, P., \& Rosen, B. P. (2002). Arsenite transport by mammalian aquaglyceroporins AQP7 and AQP9. Proceedings of the National Academy of Sciences, 99(9), 6053-6058.

Mandal, G., Mandal, S., Sharma, M., Charret, K. S., Papadopoulou, B., Bhattacharjee, H., et al. (2015). Species-specific antimonial sensitivity in Leishmania is driven by post-transcriptional regulation of AQP1. PLoS Neglected Tropical Diseases, 9(2), 0003500.

Mandal, G., Orta, J., Sharma, M., \& Mukhopadhyay, R. (2014). Trypanosomatid aquaporins: Roles in physiology and drug response. Diseases, 2(1), 3.

Mandal, G., Sharma, M., Kruse, M., Sander-Juelch, C., Munro, L. A., Wang, Y., et al. (2012). Modulation of Leishmania major aquaglyceroporin activity by a mitogenactivated protein kinase. Molecular Microbiology, 85(6), 1204-1218. 
Mukhopadhyay, R., \& Beitz, E. (2010). Metalloid Transport by Aquaglyceroporins: Consequences in the Treatment of Human Diseases MIPs and Their Role in the Exchange of Metalloids (679, 57-69)

Nasser, M. I., \& Landfear, S. M. (2004). Sequences required for the flagellar targeting of an integral membrane protein. Molecular and Biochemical Parasitology, 135(1), 89-100.

Rosen, B., \& Tamás, M. (2010). Arsenic transport in prokaryotes and eukaryotic microbes MIPs and Their Role in the Exchange of Metalloids (679, 47-55) 
VITA

\section{MANSI SHARMA}

\section{EDUCATION:}

2015 Ph.D. Candidate

Florida International University, Miami, FL, USA.

$2009 \quad$ Master of Biotechnology

University of Rajasthan, Jaipur, India

2007

Bachelor of Biotechnology

University of Rajasthan, Jaipur, India

\section{PUBLICATIONS AND PRESENTATIONS:}

Sharma, M., Mandal, G., Mandal, S., Bhattacharjee, H., and Mukhopadhyay, R. Functional role of lysine 12 in Leishmania major AQP1 (2015). Molecular and Biochemical Parasitology. Vol 201, 139-145

Mandal, G., Mandal, S., Sharma, M., Charret, K.S., Papadopoulou, B., Bhattacharjee, H., and Mukhopadhyay, R. Species-Specific Antimonial Sensitivity in Leishmania is driven by Post-Transcriptional Regulation of AQP1 (2015). Plos Neglected Tropical Diseases. Vol 10.1371/ 0003500

Mandal, G., Govindarajan, V., Sharma, M., Bhattacharjee, H., and Mukhopadhyay, R. Drug resistance in Leishmania (2014). In. Antimicrobial drug resistance: Principles and Practice for the Clinic and Bench. Eds, M. Ouellette and S. Lerner, Springer.

Mandal, G., Orta, J.F., Sharma, M., and Mukhopadhyay, R. Trypanosomatid aquaporins: Role in physiology and drug response (2014), Diseases. Vol 2, 3-23.

Mandal, G., Sharma, M., Kruse, M., Juelch, C.S., Munro, L.A., Wang, Y., Vilg, J.V., Tamás, M.J., Bhattacharjee, H.,Wiese, M., and Mukhopadhyay, R. Modulation of Leishmania major aquaglyceroporin activity by a mitogen- activated protein kinase (2012), Molecular Microbiology. Vol 85, 1204-1218.

Sharma, M., Mandal, G., Bhattacharjee, H., and Mukhopadhyay, R. $6^{\text {th }}$ Congress of European Microbiologists, Maastricht, Netherlands, June 7 - 11, 2015. Title: Posttranslational regulation of Leishmania aquaglyceroporin AQP1: crosstalk between phosphorylation and ubiquitination. 
Sharma, M., Mandal, G., Bhattacharjee, H., and Mukhopadhyay, R. Herbert Wertheim College of Medicine Research Symposium and Annual Student and Faculty Awards, Florida International University, Miami, Florida, USA, April 30 - May 1,2015. Title: Post- translational regulation of Leishmania aquaglyceroporin AQP1: crosstalk between phosphorylation and ubiquitination.

Sharma, M., Mandal, G., Bhattacharjee, H., and Mukhopadhyay, R. Kinetoplastid Molecular Cell Biology Meeting. Marine Biological Laboratory, Woods Hole, Massachusetts, USA, April 25-29, 2015. Title: Post-translational modifications of Leishmania aquaglyceroporin AQP1.

Sharma, M., Mandal, G., Bhattacharjee, H., and Mukhopadhyay, R. Graduate students appreciation week, Florida International University, Miami, Florida, USA, April 6-8, 2015. Title: Post- translational regulation of Leishmania aquaglyceroporin AQP1: crosstalk between phosphorylation and ubiquitination.

Sharma, M., Mandal, G., Bhattacharjee, H., and Mukhopadhyay, R. $17^{\text {th }}$ Annual BCI symposium, Florida International University, Miami, Florida, USA, March 26-27, 2015.Title: Post- translational regulation of Leishmania aquaglyceroporin AQP1: crosstalk between phosphorylation and ubiquitination.

Sharma, M., Mandal, G., and Mukhopadhyay, R. $16^{\text {th }}$ Annual BCI symposium, Florida International University, Miami, Florida, USA, February 13-14, 2014.Title: Posttranslational modifications of Leishmania aquaglyceroporin AQP1.

Sharma, M., Mandal, G., Mandal, S., and Mukhopadhyay, R. $5^{\text {th }}$ World Congress on Leishmaniasis. Porto de Galinhas, Pernambuco, Brazil, May 13 to 17, 2013. Title: Posttranslational regulation of Leishmania aquaglyceroporin AQP1.

Sharma, M., Mandal, G., Mandal, S., and Mukhopadhyay, R. 3rd Annual International Conference on Tropical Medicine, Florida International University, Miami, Florida, USA, February 21-22, 2013.Title: Regulation of Leishmania aquaglyceroporin AQP1 through ubiquitination. 